\title{
Simulating Structure Formation with Ultra-light Bosonic Dark Matter
}

\section{Dissertation}

zur Erlangung des mathematisch-naturwissenschaftlichen Doktorgrades

"Doctor rerum naturalium"

der Georg-August-Universität Göttingen

im Promotionsprogramm ProPhys

der Georg-August University School of Science (GAUSS)

vorgelegt von

Bodo Schwabe

aus Lübeck

Göttingen, 2018 


\section{Betreuungsausschuss}

Prof. Jens Niemeyer, Institut für Astrophysik, Universität Göttingen

Prof. Laura Covi, Institut für Theoretische Physik, Universität Göttingen

\section{Mitglieder der Prüfungskommission}

Referent:

Prof. Jens Niemeyer, Institut für Astrophysik, Universität Göttingen

Korreferent:

Dr. David Marsh, Institut für Astrophysik, Universität Göttingen

Weitere Mitglieder der Prüfungskommission:

Prof. Laura Covi, Institut für Theoretische Physik, Universität Göttingen

Prof. Ansgar Reiners, Institut für Astrophysik, Universität Göttingen

Prof. Steffen Schumann, Institut für Theoretische Physik, Universität Göttingen

Dr. Michael Wilczek, Max-Planck-Institut für Dynamik und Selbstorganisation

Tag der mündlichen Prüfung: 24.10.2018 


\section{Contents}

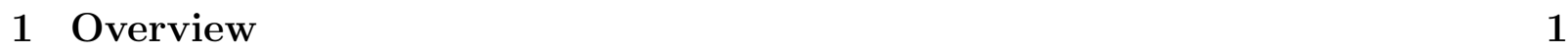

2 Fuzzy Dark Matter Cosmology

2.1 Axion-like Particles as Candidates for FDM . . . . . . . . . . . . . . . 5

2.2 FDM Evolution Equations . . . . . . . . . . . . . . . . . . . . . 6

2.3 FDM Mass Constraints . . . . . . . . . . . . . . . . . . . . . . . . . . 10

2.3 .1 CMB and LSS . . . . . . . . . . . . . . . . . . . . . . . . . . 12

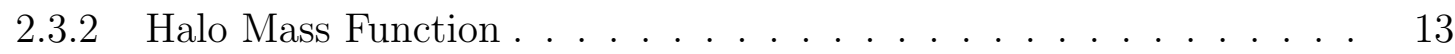

2.3 .3 UV Luminosity Function . . . . . . . . . . . . . . . . . . . . . . . . 15

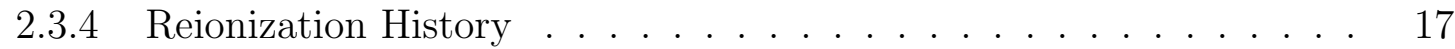

2.3 .5 Lyman-Alpha Forest . . . . . . . . . . . . . . . . . . . . . . . . . . . . . . . . . . . . . . . . .

2.3 .6 EDGES and the $21 \mathrm{~cm}$ Line . . . . . . . . . . . . . . . . . . . . . . 21

2.3 .7 Pulsar Timing Arrays . . . . . . . . . . . . . . . . . . . . . . . . . . 24

2.3 .8 Gravitational Lensing . . . . . . . . . . . . . . . . . . . . . . 26

2.3 .9 Black Hole Superradiance . . . . . . . . . . . . . . . . . . . . . . . 30

2.4 Small-Scale Tensions . . . . . . . . . . . . . . . . . . . . . . . . . 36

2.5 Halo Density Profiles . . . . . . . . . . . . . . . . . . . . . . 38

\begin{tabular}{|lll}
\hline 3 & Fuzzy Dark Matter Simulations & 41
\end{tabular}

3.1 Eulerian Grid Based Simulations . . . . . . . . . . . . . . . . . . . . . . . 41

3.2 Simulating Solitonic Cores in FDM Halos . . . . . . . . . . . . . . . . . . . 43

3.3 Self-consistent Construction of FDM Halos . . . . . . . . . . . . . . . . . . 46

3.4 Lagrangian Particle Based Simulations . . . . . . . . . . . . . . . . . . . . 47

3.5 Schrödinger-Vlasov Correspondence . . . . . . . . . . . . . . . . . . . . . . 50

3.6 Gaussian Beam Method . . . . . . . . . . . . . . . . . . . . . 53

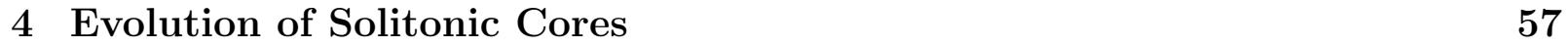

4.1 Numerical Methods . . . . . . . . . . . . . . . . . . . . 57

4.2 Binary Core Mergers . . . . . . . . . . . . . . . . . . . . . . . . . . . 59

4.3 Core-Halo Mass Relation . . . . . . . . . . . . . . . . . . . . . . . . . . . . 69

4.4 Mergers of Multiple Cores . . . . . . . . . . . . . . . . . . . . . . . 69

4.5 Tidal Disruption of Subhalo Cores . . . . . . . . . . . . . . . . . . . . . . . . . . . 72

4.6 Relativistic Axion Stars . . . . . . . . . . . . . . . . . . . . . . . . . 74 
$\begin{array}{|lll|}5 & \text { Gaussian Beam Method in Fuzzy Dark Matter Simulations } & 77\end{array}$

5.1 Implementation of the Gaussian Beam Method . . . . . . . . . . . . . . . . 77

5.2 Numerical Tests . . . . . . . . . . . . . . . . . . . . . . . . 79

5.3 WKB approximation . . . . . . . . . . . . . . . . . . . . . . . . . . . . . . . . . . . 80

5.4 Spherical Collapse . . . . . . . . . . . . . . . . . . . . . . . . . . . . . . . . . . . . . . . 81

5.5 Cosmological Simulation . . . . . . . . . . . . . . . . . 86

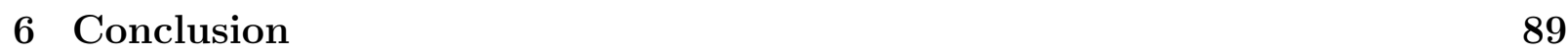

\begin{tabular}{|rr}
\hline Acknowledgements & 124
\end{tabular} 


\section{List of Figures}

2.1 Summary of FDM mass constraints from various observational techniques together with the preferred mass range from dwarf galaxy phenomenology within the Milky Way assuming that FDM constitutes all DM. . . . . . . . 11

4.1 Head-on collision of two cores with mass ratio $\mu=2$ and high relative velocity. Upper panels: density profiles at different times for relative phases $\Phi=0$ (left) and $\Phi=\pi$ (right) along the symmetry axis. Numerical results are shown for the initial and final state as well as for the time of maximal interference. For comparison, we plot the interference pattern predicted from|Equation 4.7 at the same time. Deviations can be attributed mostly to a small offset in the time of maximal interference. Lower panels: mass and energy contributions. Total energy and mass are conserved, while kinetic energy associated to the cores' relative motion $\left(K_{v}\right)$ is transferred into the interference pattern yielding large values of $K_{\rho}$ during the interaction. The equality of the lower panels shows the independence of the evolution with respect to the initial phase shift $\Phi .6$. . . . . . . . . . . . . . . . 60

4.2 Mass, energy and angular momentum evolution of two representative binary collisions with initial values $\mu=1, v_{\|}=0 \mathrm{~km} / \mathrm{s}$, and $L_{z}=2.4 \times 10^{4} M_{\odot} \mathrm{Mpc}$ $\mathrm{km} / \mathrm{s}$ (rescaled by $10^{2}$ ). Cores with equal phase $(\Phi=0)$ immediately merge (left). In perfect phase opposition $(\Phi=\pi)$, the two cores first mutually repel each other multiple times before merging (right). The bounces are indicated by black arrows. The emerging cores are excited as seen by the oscillations of gradient and gravitational energy, $K_{\rho}$ and $W$. The loss of total mass, energy, and angular momentum results from matter absorption inside the sponge. . . . . . . . . . . . . . . . . . . 62

4.3 Evolution of the core (solid lines) and total (dashed lines) mass for binary mergers. The triplets identify the point $\left(\mu, \Phi, L_{z}\right)$ in parameter space. An-

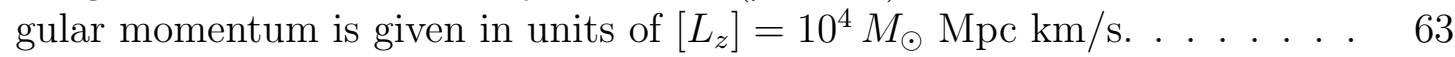

4.4 Volume rendered images of two representative binary mergers in phase (top) and with opposite phase (bottom) showing the central region of the computational domain at $t=0.7, t=0.94, t=2.0$ and $t=7.0$ in $\mathrm{Mpc} / \mathrm{km}$

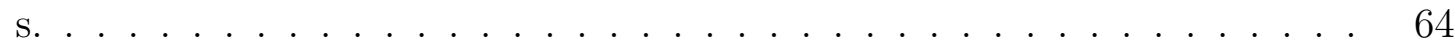


4.5 Slice through the symmetry plane of a representative ellipsoid. Its density is color-coded while arrows denote the strength and direction of its velocity field. It roughly forms closed elliptical orbits. . . . . . . . . . . . . . .

4.6 Binary mergers with different mass ratios $\mu$. Left: evolution of the core mass of the more massive core. Right: final radial density profiles. Solid lines represent fitted core profiles as defined in|Equation 3.4. The black line corresponds to $r^{-3}$ as expected for the outer parts of an NFW profile. . . . $\quad 66$

4.7 Core mass as a function of the total energy and mass. The star indicates the relation for a single solitonic core. Run 1 denotes the simulations with almost equal total energy for different angular momenta and phases. Runs 2 and 3 show the dependence on mass ratio $\mu$ and total energy $E$, respectively. Multiple core mergers are shown as run 4 (cf. section 4.4). See main text for details. . . . . . . . . . . . . . . . . . . . . . . . . . 67

4.8 Final radial density profiles for all conducted multimerger runs. Solid lines represent fitted core profiles as defined in $\mid$ Equation 3.4 $\mid$ The black line corresponds to $r^{-3}$ as expected for the outer parts of an NFW profile. . . . 71

4.9 Density distribution of a multimerger simulation with 13 halos at different times.

5.1 Gravitational collapse of a spherical overdensity at different redshifts $z=$ $\{4.0 ; 3.5 ; 3.0 ; 2.0\}$. From top to bottom the density obtained from our (1st row) finite difference, (2nd row) Gaussian beam, and (3rd row) N-body scheme are shown together with the evolution of the corresponding phase integrated within the (4th row) finite difference, and (5th row) Gaussian beam method. It can be seen that the GBM and the finite difference scheme initially yield comparable results. The collapse integrated with the GBM slightly lags behind the one from the finite difference method. The two methods start to significantly deviate after the formation of a solitonic core in the center at $z=3.0$. Density scales are identical in all plots, while phases

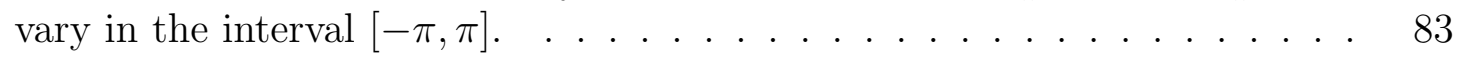

5.2 Shown are slice plots through the final density profiles after gravitational collapse of a spherical overdensity evolved from redshift $z=100$ to $z=7$ with various numerical methods as indicated by plot labels. The corresponding final radial density profiles are also shown. They match well in the outer halo while strongly deviating in the core region. Since we average over radial density shells, we expect the profiles obtained by employing the GBMs to coincide with the N-body density profile by means of the SchrödingerVlasov correspondence. It is interesting to see that the GBM with WKB approximation fits the N-body profile significantly better than the standard

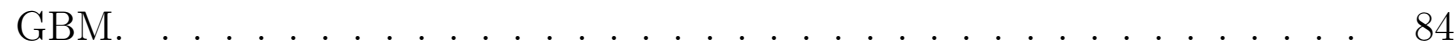


5.3 Evolution of the complex phase during spherical collapse of a Gaussian overdensity with zero initial momentum at different redshifts $z=\{100,50,7\}$. Top panels show results obtained by the standard GBM, while lower panels were produced with the WKB approximated GBM. The similarity between both phases verifies the correct implementation of both methods and their suitability for the simulation of gravitational collapse. Importantly, the phase is consistently reconstructed by both methods even though it is badly resolved. This has to be contrasted with finite difference approaches needing highly resolved phases. By construction, the initial phase within the WKB approximated GBM is globally zero. Within the standard GBM this is only true in the central regions, where beams overlap enough in order to reproduce the correct phase. . . . . . . . . . . . . . . . . 85

5.4 Initial power spectra at $z=300$ as obtained from AxionCAMB (black) and within Enzo after wavefunction construction from MUSIC generated phasespace sampling (red) and after reconstruction from Gaussian beams (orange). See text for details. . . . . . . . . . . . . . . . . . 87 


\title{
Nomenclature
}

\author{
ALP Axion-Like Particle \\ AMR Adaptive Mesh Refinement \\ BEC Bose-Einstein Condensate \\ BH Black Hole \\ CDM Cold Dark Matter \\ CMB Cosmic Microwave Background \\ CP Charge-Parity \\ DM Dark Matter \\ dSph dwarf Spheroid \\ FBI Fourier-Bros-Iagolnitzer \\ FDM Fuzzy Dark Matter \\ GBM Gaussian Beam Method \\ HI neutral hydrogen \\ HII ionized hydrogen \\ HMF Halo Mass Function \\ IGM Intergalactic Medium \\ ISW Integrated Sachs-Wolf \\ kSZ kinetic Sunyaev-Zel'dovich \\ LSS Large-Scale Structure \\ Ly $\alpha$ Lyman-Alpha
}


NFW Navarro-Frenk-White

QCD Quantum Chromodynamics

RK4 fourth-order Runge-Kutta

SP Schrödinger-Poisson

UV Ultra-Violet

UVLF Ultra-Violet Luminosity Function

WDM Warm Dark Matter

WIMP Weakly Interacting Massive Particle

WKB Wentzel-Kramers-Brillouin 


\section{Chapter 1}

\section{Overview}

With experimental data from missions like Planck Planck Collaboration, 2018], SDSS [SDSS Collaboration, 2012], Euclid [Euclid Collaboration, 2013], HETDEX [Hill et al., 2008], Gaia [Gaia Collaboration, 2018] and many more mapping the cosmic microwave background (CMB), cosmic web and the Milky Way, cosmology has entered an era of high precision physics. With large numerical simulations, including the Millenium-II BoylanKolchin et al., 2009], IllustrisTNG [Springel et al., 2017], EAGLE [McAlpine et al., 2016] and FIRE [Hopkins et al. 2018] collaborations, theoretical models can be tested against observations with ever higher accuracy. In this way the standard model of cosmology has been established, which quantifies the different energy density budgets of the universe. Joined observations of photons, gravitational waves and neutrinos have transformed cosmology into a multi-messenger research area The LIGO Scientific Collaboration and The Virgo Collaboration, 2017; The IceCube Collaboration, 2018].

Despite being a cornerstone of the standard model, the exact nature of dark matter (DM) remains unknown. The lack of evidence for weakly interacting massive particles (WIMPs) that could constitute DM is generating increasing interest in alternative candidates. These include the theoretically well-motivated QCD axion [Peccei and Quinn, 1977], which solves the strong CP problem, and axion-like particles (ALPs) generically arising in string theories Arvanitaki et al., 2010; Hui et al., 2017. This thesis focuses on the latter. While indistinguishable from cold dark matter (CDM) on large scales, these ultra-light (pseudo-)scalar particles are thought to form a coherent state on galactic scales. Wave effects then induce a Jeans scale $r_{J}$ below which gravitational collapse is suppressed $[\mathrm{Hu}$ et al. 2000.

If non-gravitational interactions can be neglected, this form of DM is known as fuzzy dark matter (FDM) $\mathrm{Hu}$ et al., 2000. If coherently produced due to e.g. the misalignment mechanism or evolved to a Bose-Einstein condensate (BEC), it can be mathematically described by a massive scalar field $\Psi(t, x)$ whose evolution is consequently governed by the Klein-Gordon equation with a potential reflecting the metric back reaction defined via the Einstein equation.

On sub-horizon scales, and in the Newtonian gauge, growth of density perturbations is suppressed on small scales due to an effective sound speed that is not present in standard 
CDM. For masses $m \lesssim 10^{-22} \mathrm{eV}$ structure formation is suppressed to a degree that is inconsistent with large-scale structure data. Slightly weaker constraints can be inferred from alterations in the cosmic microwave background (CMB) power spectrum.

Using initial conditions compatible with CMB data, structures are numerically found to form hierarchically by the merging of increasingly larger local overdensities. A suppression on small scales thus delays the collapse to DM halos and the subsequent galaxy and star formation in their potential wells. This shifts reionization to lower redshifts and alters the optical depth and gravitational lensing potential of the CMB.

Since deviations between FDM and CDM are larger on scales below $r_{J}$, stronger bounds on $m$ can be expected from observables quantifying the distribution of DM overdensities on galactic scales. These include Lyman- $\alpha$ and $21 \mathrm{~cm}$-line spectra that measure the energy spectrum of neutral hydrogen. Gravitational (micro-)lensing and stellar kinematics measurements provide data to construct the halo mass function, subhalo mass function and the luminosity function for various redshifts. Since the effective sound speed leads to a steep increase in the critical overdensity needed for collapse on scales below $r_{J}$ and consequently to a sharp cutoff in the halo mass function below a minimum mass, these measurements can probe FDM masses $m \lesssim 10^{-21} \mathrm{eV}$.

On even smaller scales, the observation, or non-observation, of the formation and binary mergers of solitons and soliton neutron star encounters can provide stringent constraints on the FDM mass and hypothetical non-gravitational interactions. Furthermore, FDM in the vicinity of a rotating black hole with mass $M$ can efficiently decrease the black hole's angular momentum via superradiance if $m M \sim 1$, in natural units. The observation of spinning black holes within a wide range of masses can thus significantly reduce the possible parameter space for FDM. In addition, DM constraints from direct and indirect DM detection experiments rely heavily on the predicted local DM distribution.

As the model dependent phenomena on (sub-)galactic scales are due to highly non-linear dynamics, numerical simulations are of paramount importance in order to make stringent model-dependent predictions that can discriminate between different DM theories. This thesis contributes to the endeavor of linking theoretical FDM models to astrophysical observations by defining and quantifying phenomenological predictions.

In doing so, the author utilized different numerical schemes in order to discretize the Schrödinger-Poisson (SP) system which governs the non-relativistic, Newtonian dynamics of FDM. These include finite difference methods [Schwabe et al., 2016], pseudo spectral methods Du et al. 2018 and Lagrangian fluid descriptions with FDM initial conditions Veltmaat et al., 2018]. All three numerical approaches will be extensively discussed below focussing on their advantages and disadvantages as well as on their various results from cosmological to sub-galactic scales. The overall aim of these simulations is to constrain the FDM mass $m$ as the single parameter of the theory.

The thesis is organized as follows. In chapter 2 ALPs are introduced as well motivated candidates for FDM and important evolution equations for FDM are summarized. We then present a detailed discussion on the above outlined FDM mass constraints.

FDM simulations and their results are summarized in chapter 3. They include a novel hybrid simulation method that combines the efficiency of Lagrangian n-body schemes on 
cosmological scales with the precision of finite difference methods for pre-selected FDM halos. The author and his collaborator Jan Veltmaat equally contributed to the code development, while the subsequent numerical simulations were conducted by the latter. The results are presented in a publication printed in Physical Review D Veltmaat et al. 2018]. The simulated FDM halos are found to have a granular structure on de Broglie scales while, on average, NFW profiles, as obtained from CDM simulations, are recovered. This numerically confirms the Schrödinger-Vlasov correspondence as discussed below, which is only broken by the formation of a bound solitonic core in central halo regions. The main numerical challenge is the construction of appropriate initial and boundary conditions between the two schemes. Discretizing the problem initially in an overcomplete set of Gauss kernels and evolving them separately, assuming classical Newtonian gravitation, has the potential to significantly improve them or even replace the finite difference scheme on intermediate scales.

Chapter 4 describes the evolution of these solitonic cores. The numerical results from binary and multi-soliton mergers constitute the first half of this thesis and were predominantly obtained by the author. Initial code development and the publication in Physical Review D were done in collaboration with Jens C. Niemeyer and Jan F. Engels [Schwabe et al., 2016]. The results were subsequently confirmed and extended by Mocz et al. [2017]. They were utilized to semi-analytically show that the numerically found relation between the core and halo mass can be seen as a consequence of the merger history of FDM halos [Du et al. 2017]. The analysis was supported by the author of this thesis, who also helped in writing the final draft. Finally, tidal disruption of FDM subhalo cores were investigated by Du et al. [2018], where the author of this thesis again contributed to the final version.

A detailed status report on the ongoing application of the so-called Gaussian Beam Method (GBM) to FDM cosmology simulations is given in chapter 5. Numerical tests including spherical collapse simulations in an expanding universe already show promising results motivating further research in the future.

We conclude the thesis in chapter 6 and outline promising directions for future numerical investigations of FDM cosmologies. 


\section{Chapter 2}

\section{Fuzzy Dark Matter Cosmology}

In this thesis, DM is assumed to consist solely of ultralight scalar particles with mass $m \sim 10^{-22} \mathrm{eV}$ and negligible self-interaction. This sort of DM is commonly referred to as fuzzy dark matter (FDM). Since thermally produced DM below the keV scale is excluded by observations of structure formation due to its excessive free streaming length, these bosonic particles need to have a non-thermal production mechanism, like misalignment in axion theories, in the early universe that creates them at approximately zero temperature, or vanishing velocity dispersion. Owing to their extremely light masses, their cold initial state and large average energy density $\Omega_{\mathrm{FDM}} h^{2} \sim 0.12$, occupation numbers within de Broglie wavelength volumes are extraordinary high. This motivates us to treat the collection of particles in a mean field limit as a non-relativistic scalar field under the influence of selfgravity [Marsh, 2016b].

Standard CDM is evolved as a pressureless fluid in phase-space using the Vlasov-Poisson system of equations whose first moments give rise to the Euler equations. They are implemented in N-body cosmology codes like Gadget. FDM is different in that it is treated as a non-relativistic scalar field in position space. Its evolution is therefore governed by the Schrödinger-Poisson (SP) system. Since the Schrödinger equation admits wave-like behaviour on de Broglie scales, FDM is expected to differ strongly from CDM on these scales as the latter does not have any characteristic length scale [Hu et al., 2000].

After establishing axion-like particles as well motivated candidates for FDM, we collectively present the evolution equations relevant for this thesis. We continue with a summary of FDM mass constraints from cosmological observations, review small-scale tensions between CDM observations and simulations and end this section with a presentation of FDM imprints in halo density profiles.

\subsection{Axion-like Particles as Candidates for FDM}

Axions have originally been introduced to solve the strong CP problem of quantum chromodynamics that stems from the CP violating part of the QCD Lagrangian $\mathcal{L} \supset \theta \tilde{F}_{\mu \nu} F^{\mu \nu}$ that is not forbidden by any symmetry within the standard model of particle physics Peccei 
and Quinn, 1977]. It arises due to the $\theta$-vacua of QCD [Coleman, 1985. The experimental constraint $\theta<10^{-10}$ suggests an extremely fine-tuned cancellation between the bare angle $\tilde{\theta} \in[0,2 \pi]$ and the argument of the quark mass matrices' determinant Baker et al., 2006]

$$
\theta=\tilde{\theta}+\arg \operatorname{det} M_{u} M_{d} \lesssim 10^{-10},
$$

or a mechanism to dynamically drive the parameter $\theta$ to zero. The Peccei and Quinn solution to the strong CP problem is to consider $\theta$ to be the pseudo Nambu Goldstone boson of a global $U(1)$ symmetry that is spontaneously broken at an energy scale $f_{a}$ Peccei and Quinn, 1977. At a lower scale $\mu<f_{a}$, QCD instantons can then non-perturbatively induce a potential that drives $\theta$ to zero, thus dynamically restoring CP conservation Vafa and Witten, 1984. The corresponding quantum excitation is the so called (QCD-)axion.

Similarly, axions appear in string theory compactifications as Kaluza-Klein zero modes of antisymmetric tensor fields [Witten, 1984]. Due to their complex topology, realistic string theories indeed feature a multitude of axions over a wide mass range Arvanitaki et al., 2010; Kreuzer and Skarke, 2002; Svrcek and Witten, 2006; Svrcek, 2006; He, 2013; Hui et al., 2017]. These axions are well motivated candidates for FDM.

\subsection{FDM Evolution Equations}

FDM is modeled as a self-gravitating scalar field $\phi$ with mass $m$. Its action is given by

$$
S=\frac{1}{16 \pi G} \int \mathrm{d}^{4} x \sqrt{-g} R-\int \mathrm{d}^{4} x \sqrt{-g} \mathcal{L}_{m}
$$

with Lagrangian density $(c=1)$

$$
\mathcal{L}_{m}=\frac{1}{2} g^{\mu \nu} \nabla_{\mu} \phi^{*} \nabla_{\nu} \phi+\frac{1}{2} \frac{m^{2}}{\hbar^{2}}|\phi|^{2}
$$

The corresponding Euler-Lagrange equations (equations of motion) are the Klein-Gordon equation

$$
\left(\square-\frac{m^{2}}{\hbar^{2}}\right) \phi=\left(g^{\mu \nu} \nabla_{\mu} \nabla_{\nu}-\frac{m^{2}}{\hbar^{2}}\right) \phi=\left(\frac{1}{\sqrt{-g}} \partial_{\mu}\left[\sqrt{-g} g^{\mu \nu} \partial_{\nu}\right]-\frac{m^{2}}{\hbar^{2}}\right) \phi=0
$$

and the Einstein equations

$$
R_{\mu \nu}-\frac{1}{2} g_{\mu \nu} R=8 \pi G T_{\mu \nu}
$$

with energy-momentum tensor

$$
T_{\mu \nu}=\frac{2}{\sqrt{-g}} \frac{\delta\left(\sqrt{-g} \mathcal{L}_{m}\right)}{\delta g_{\mu \nu}}=\nabla_{\mu} \phi \nabla_{\nu} \phi-\frac{1}{2} g_{\mu \nu}\left(g^{\rho \sigma} \nabla_{\rho} \phi \nabla_{\sigma} \phi+\frac{m^{2}}{\hbar^{2}} \phi\right) .
$$


We are considering the perturbed Friedmann-Lemaître-Robertson-Walker line element in Newtonian gauge

$$
d s^{2}=-(1+2 V) \mathrm{d} t^{2}+a^{2}(1-2 U)\left(\mathrm{d} x^{2}+\mathrm{d} y^{2}+\mathrm{d} z^{2}\right)
$$

Here, $V$ is the Newtonian gravitational potential, which satisfies the Poisson equation

$$
\nabla^{2} V=4 \pi G a^{2} \delta \bar{\rho}
$$

where $\bar{\rho}$ is the energy density of the axion field $\phi$. We assume vanishing anisotropic stress. Then, the Einstein equations yield $V=U$ and we have

$$
\bar{\rho}=-T_{0}^{0}=\frac{1}{2}\left[(1-2 V) \dot{\phi}^{2}+\frac{m^{2}}{\hbar^{2}} \phi^{2}+a^{-2}(1+2 V) \partial^{i} \phi\right]+\mathcal{O}\left(V^{2}\right) .
$$

As in Seidel and Suen [1990], we assume the mass of the scalar field to be a quantity of order unity, whereas the wave number $k$ of the field is of order $\epsilon_{\mathrm{NR}}$. Furthermore, $V \sim \epsilon_{\mathrm{NR}}^{2}$ in the post Newtonian limit Weinberg, 1972]. The fully relativistic theory has been worked out by Suárez and Chavanis [2015], showing that the relativistic corrections are small for structure formation during matter domination. Under the above assumptions, (2.4) reads

$$
\left[-(1-2 V)\left(\partial_{t}^{2}+3 H \partial_{t}\right)+a^{-2}(1+2 V) \nabla^{2}-\frac{m^{2}}{\hbar^{2}}+\mathcal{O}\left(\epsilon_{\mathrm{NR}}^{4}\right)\right] \phi=0
$$

Neglecting the subdominant potential $V \ll 1$, it implies a dispersion relation

$$
\omega=\sqrt{\frac{m^{2}}{\hbar^{2}}+\frac{k^{2}}{a^{2}}}=\frac{m}{\hbar}+\frac{k^{2} \hbar}{2 a^{2} m}+\mathcal{O}\left(\epsilon_{\mathrm{NR}}^{4}\right) .
$$

Since the frequency $\omega$ is constant to leading order we can use the WKB theory in the limit $H / m \sim \epsilon_{\text {WKB }}$ when the axion field behaves like CDM. We can then use the ansatz solution Widrow and Kaiser, 1993:

$$
\phi=\frac{\hbar}{\sqrt{2} m} \bar{\psi} e^{-i m t / \hbar}
$$

where $\bar{\psi}$ is only slowly varying with time $\left(|\hbar \dot{\bar{\psi}}| /|m \bar{\psi}| \sim \epsilon_{\mathrm{WKB}}\right)$. If the field $\phi$ is real, we have to consider only the real part of the equation or equivalently have to add the complex conjugate in the above ansatz. Performing a double expansion to $\mathcal{O}\left(\epsilon_{\mathrm{NR}, \mathrm{WKB}}^{2}\right)$, the nonrelativistic limit of the Klein-Gordon equation (2.10) gives the equation for the amplitude $\bar{\psi}$ Marsh, 2015]:

$$
i \hbar \partial_{t} \bar{\psi}-\frac{3 i \hbar H}{2} \bar{\psi}+\frac{\hbar^{2}}{2 m a^{2}} \nabla^{2} \bar{\psi}-m V \bar{\psi}=0
$$


Independently, the conjugate equation applies to $\bar{\psi}^{*}$. Moreover, taking the same limits, (2.9) becomes

$$
\bar{\rho}=|\bar{\psi}|^{2}+\mathcal{O}(\epsilon)
$$

Note that $\bar{\psi}$ is the physical field. It is related to the comoving field $\psi \equiv \bar{\psi} a^{3 / 2}$ obeying $|\psi|^{2}=\bar{\rho} a^{3} \equiv \rho$ and the comoving Schrödinger equation

$$
i \hbar \partial_{t} \psi=-\frac{\hbar^{2}}{2 a^{2} m} \nabla^{2} \psi+m V \psi \text {. }
$$

Using Madelung's transformation Madelung, 1927

$$
\psi=\sqrt{\rho} e^{i S / \hbar}
$$

the imaginary part of the Schrödinger Equation 2.15 yields

$$
\partial_{t} \sqrt{\rho}=-\frac{1}{2 m a^{2}}\left[2(\nabla \sqrt{\rho}) \cdot(\nabla S)+\sqrt{\rho} \nabla^{2} S\right] .
$$

Additionally defining the fluid velocity

$$
\mathbf{v}=\nabla \frac{S}{m}
$$

we can cast 2.17) into a comoving continuity equation

$$
\partial_{t} \rho+\frac{1}{a^{2}} \nabla \cdot(\rho \mathbf{v})=0
$$

Similarly, the real part of 2.15 becomes the comoving quantum Hamilton-Jacobi equation Bohm, 1952

$$
\partial_{t} S=-\left[\frac{(\nabla S)^{2}}{2 a^{2} m}+m V+Q\right] .
$$

It differs from the classical comoving Hamilton-Jacobi Equation 3.42 only by the Bohm quantum potential

$$
Q=-\frac{\hbar^{2}}{2 m a^{2}} \frac{\nabla^{2} \sqrt{\rho}}{\sqrt{\rho}},
$$

which depends on the curvature of the amplitude of the wavefunction. Thus, $\mathbf{v}$ as defined above can again be interpreted as the fluid velocity Bohm, 1952. We will refine these arguments below. Spatial differentiation of Equation 2.20 then yields the force equation

$$
\partial_{t} \mathbf{v}+\frac{1}{a^{2}} \mathbf{v} \cdot \nabla \mathbf{v}+\nabla V-\frac{\hbar^{2}}{2 m^{2} a^{2}} \nabla\left(\frac{\nabla^{2} \sqrt{\rho}}{\sqrt{\rho}}\right)=0 .
$$


If linearized, Equation 2.19 and Equation 2.22 become

$$
\begin{aligned}
& 0=a^{2} \partial_{t} \delta \rho+\rho_{0} \nabla \cdot \mathbf{v}, \\
& 0=\rho_{0} \partial_{t} \mathbf{v}+\rho_{0} \nabla V-\frac{\hbar^{2}}{4 m^{2} a^{2}} \nabla\left(\nabla^{2} \delta \rho\right),
\end{aligned}
$$

where $\delta \rho=\rho-\rho_{0}$ denotes the comoving density perturbation above the constant background $\rho_{0}$. Taking the time derivative of the first equation and the spatial derivative of the second one, we can combine these equations with the Poisson equation

$$
\nabla^{2} V=\frac{4 \pi G}{a} \delta \rho=\frac{3 H_{0}^{2}}{2 a} \Omega_{\mathrm{FDM}} \frac{\delta \rho}{\rho_{0}}
$$

where $\Omega_{\mathrm{FDM}}$ is the current FDM density parameter. The result is a second-order differential equation for $\delta \rho$ :

$$
\partial_{t} a^{2} \partial_{t} \delta \rho-\frac{3 H_{0}^{2}}{2 a} \Omega_{\mathrm{FDM}} \delta \rho+\frac{\hbar^{2}}{4 m^{2} a^{2}} \nabla^{2} \nabla^{2} \delta \rho=0 .
$$

Spatial Fourier transformation of $\delta \rho$ then yields

$$
\partial_{t} a^{2} \partial_{t} \rho_{k}-\frac{3 H_{0}^{2}}{2 a} \Omega_{\mathrm{FDM}} \rho_{k}+\frac{\hbar^{2} k^{4}}{4 m^{2} a^{2}} \rho_{k}=0
$$

As in Woo and Chiueh [2009], assuming a FDM dominated universe with $\Omega_{\mathrm{FDM}}=1$ we have $a=\left(t / t_{0}\right)^{2 / 3}$ and therefore $H=2 /(3 t)$. Defining $x=\hbar k^{2} /\left(m H_{0} \sqrt{a}\right)$ yields

$$
\frac{d t}{d x}=-\frac{3 m H_{0} t_{0}}{\hbar k^{2}} a^{2}
$$

Thus, in an FDM dominated universe, 2.27) is equivalent to

$$
\frac{d^{2}}{d x^{2}} \rho_{k}+\omega^{2} \rho_{k}=0 \quad \text { with } \quad \omega^{2}=1-\frac{6}{x^{2}} .
$$

The solution to 2.29) is given by Woo and Chiueh, 2009:

$$
\rho_{k}(x)=c_{1}\left(\frac{3 \sin x-x^{2} \sin x-3 x \cos x}{x^{2}}\right)+c_{2}\left(\frac{3 \cos x-x^{2} \cos x+3 x \sin x}{x^{2}}\right),
$$

where $c_{1}, c_{2}$ are constants of integration that need to be fixed by appropriate initial conditions. On the one hand, for $x^{2} \geq 6, \omega^{2}$ is positive, and Equation 2.29 yields an oscillatory solution corresponding to a propagating sound wave with effective sound speed $\mathrm{Hu}$ et al. 2000; Hwang and Noh, 2009

$$
c_{\mathrm{eff}}^{2}=\frac{\hbar^{2} k^{2} / 4 a^{2} m^{2}}{1+\hbar^{2} k^{2} / 4 a^{2} m^{2}} .
$$


On the other hand, for $x^{2} \leq 6$, we have an increasing or decreasing solution. In this case, the support from the correction $Q$ in Equation 2.21 is no longer strong enough to prevent gravitational instability. The boundary $x^{2}=6$ defines the Jeans wave number in the linear regime Khlopov et al. 1985

$$
k_{J}=(6 a)^{1 / 4}\left(\frac{m H_{0}}{\hbar}\right)^{1 / 2}
$$

and the corresponding Jeans length

$$
r_{J}=\frac{2 \pi a}{k_{J}}=\left(\frac{8}{3}\right)^{1 / 4} \pi \sqrt{\frac{\hbar}{m H_{0}}} a^{3 / 4}=a^{3 / 4} \hbar^{1 / 2} \pi^{3 / 4}\left(G \rho_{0}\right)^{-1 / 4} m^{-1 / 2} .
$$

Setting $a=\hbar=1$, this exactly coincides with the Jeans length found by $\mathrm{Hu}$ et al. [2000], which was derived directly from the Schrödinger equation. It is then argued that the Jeans length can be interpreted as the de Broglie wavelength

$$
\lambda=2 \pi \hbar(m v)^{-1} \simeq \sqrt{\frac{12}{32}} \pi^{3 / 2} \hbar m^{-1}(G \rho)^{-1 / 2} r^{-1}=\sqrt{\frac{12}{32}} \pi^{3 / 2} a^{3 / 2} \hbar m^{-1}\left(G \rho_{0}\right)^{-1 / 2} r^{-1}
$$

of the ground state of a particle in a potential well if all scales fall together $(\lambda \simeq r \Leftrightarrow$ $\left.\lambda \simeq r_{J}\right)$. Then, stability below the Jeans length is ensured by gradient energy. Above we assumed that the free-fall time

$$
t_{\mathrm{ff}}=\sqrt{\frac{3 \pi}{32 G \rho}}
$$

of a spherically symmetric distribution of mass is the relevant dynamical time scale. Note that $r_{J} \propto \rho_{0}^{-1 / 4}$. When investigating soliton solutions of the Schrödinger equation, we will find the same scaling relation. Note further that in the classical limit $(Q \rightarrow 0)$ appropriate for ordinary CDM, Equation 2.29 reduces to

$$
\frac{d^{2}}{d x^{2}} \rho_{k}-\frac{6}{x^{2}} \rho_{k}=0
$$

and therefore $\rho_{k}=x^{-2}$ ( $\rho_{k}=x^{3}$ is a solution as well but not square-integrable). In this case we have gravitational instability even on the smallest scales.

\subsection{FDM Mass Constraints}

The goal of FDM research is to constrain the mass $m$ of the underlying boson as the only free parameter of the system. Assuming a homogeneous background, Equation 2.10 reduces to

$$
\left[\partial_{t}^{2}+3 H \partial_{t}+\frac{m^{2}}{\hbar^{2}}\right] \phi=0
$$




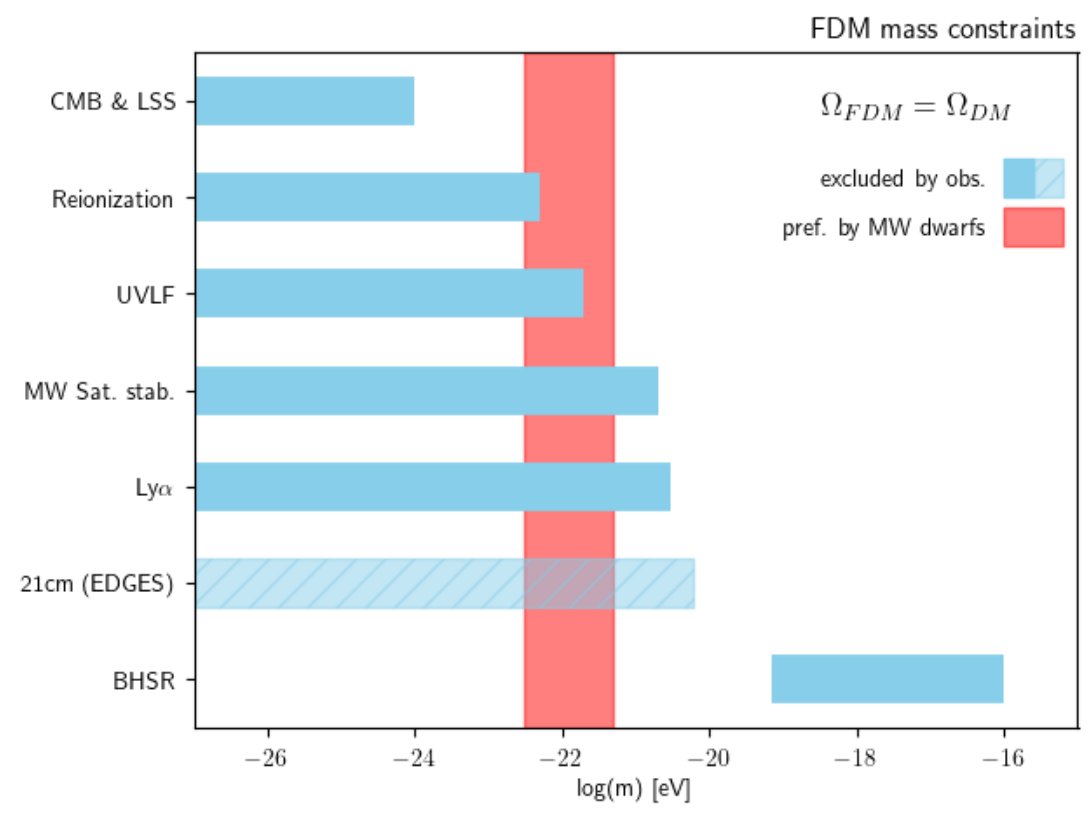

Figure 2.1: Summary of FDM mass constraints from various observational techniques together with the preferred mass range from dwarf galaxy phenomenology within the Milky Way assuming that FDM constitutes all DM.

This defines the dynamics of a damped harmonic oscillator. We therefore infer two very different limiting cases. If $m<H$, the system is overdamped. The homogeneous energy density and pressure

$$
\begin{aligned}
& \rho=\frac{\left(\partial_{t} \phi\right)^{2}}{2 a^{2}}+\frac{m^{2} \phi^{2}}{2} \\
& P=\frac{\left(\partial_{t} \phi\right)^{2}}{2 a^{2}}-\frac{m^{2} \phi^{2}}{2}
\end{aligned}
$$

imply a dark energy component with equation of state $\omega=P / \rho=-1$ and constant energy density for a slowly rolling field $\partial_{t} \phi \simeq 0$. Only for $m>H$ is the field oscillating around $\omega=$ 0 and its energy density drops as $a^{-3}$ making it a viable DM candidate [Turner, 1983 ; Press et al., 1990; Hložek et al., 2015. Particles with masses $m \leq H_{0} \sim 10^{-33} \mathrm{eV}$ still behave as dark energy today and are thus mainly unconstrained as long as the expansion history does not depart too far from standard $\Lambda \mathrm{CDM}$ cosmology. Similarly, for masses $m>10^{-20} \mathrm{eV}$ the corresponding Jeans scale (cf. Equation 2.33) becomes so small that FDM is almost indistinguishable from CDM. Within this wide mass range there is a plethora of possible observational phenomena that can help to discriminate between standard CDM and FDM cosmologies. They are summarized in this section. The derived FDM mass constraints are collectively shown in Figure 2.1 together with the preferred mass range from dwarf galaxy phenomenology within the Milky Way. 


\subsubsection{CMB and LSS}

The effects of a FDM component on the two-point temperature auto-correlation power spectrum of the CMB have been investigated by Hložek et al. [2015]. In order to ensure structure formation on all scales, matter-radiation equality is fixed to its standard value $z_{e q} \sim 3000$ in all considered scenarios by appropriately lowering $\Omega_{C D M}$ for $m \geq H\left(z_{e q}\right) \sim$ $10^{-27} \mathrm{eV}$. Otherwise, FDM starts oscillating after matter-radiation equality and therefore contributes to dark energy at $z_{e q}$. For $m \geq H\left(z_{e q}\right)$, FDM behaves like matter during the entire matter-dominated era. It therefore only alters higher order peaks of the power spectrum. FDM with $m \leq H\left(z_{e q}\right)$ has stronger effects on the CMB power spectrum. Keeping the current Hubble rate $H_{0}$ fixed, a non-negligible FDM fraction reduces the contributions from a cosmological constant by implying an enhanced current DM energy density. This in turn reduces the inferred age of the universe and thus the distance to the surface of last scattering. The consequentially increased angular size of the sound horizon $\theta_{A}$ results in a shift of the locations of the CMB acoustic peaks to lower angles. Additionally, the late time evolution is altered enhancing the integrated Sachs-Wolf (ISW) plateau. Instead of fixing $H_{0}$, we can also fix the position of the acoustic peaks to their standard values. Deviations are than mainly restricted to the ISW plateau which is less well restricted due to cosmic variance. Keeping the acoustic peaks fixed requires a significant reduction in $H_{0}$.

The large-scale structure of the universe (LSS) can be used to constrain the matter power spectrum assuming that galaxies trace DM perturbations with a scale-dependent bias. In the absence of dedicated numerical simulations for FDM, the bias has to be approximated analytically [LoVerde, 2014, Hložek et al., 2015]. As before, Hložek et al. [2015] obtain constraints by either fixing $\theta_{A}$ or $H_{0}$, while appropriately changing the other with varying FDM fraction. Keeping $H_{0}$ fixed again implies a reduced age of the universe. The power spectrum is therefore lower on all scales since perturbations have less time to grow. The shape of the power spectrum is only altered for $m \geq H\left(z_{e q}\right)$. Changing instead $H_{0}$ considerably alters the power spectrum emphasizing the complementarity between CMB and LSS data.

Refining the analysis of Amendola and Barbieri [2006], Hložek et al. [2015] investigate CMB data gathered by the Planck [Planck Collaboration, 2014] and WMAP missions WMAP Collaboration, 2013 complemented by the high angle data sets provided by the Atacama Cosmology Telescope [ACT Collaboration, 2014] and the South Pole Telescope George et al., 2015 in order to constrain the two-dimensional parameter space. Additionally, they compare FDM matter power spectra obtained with AxionCAMB with the observed ones from the WiggleZ survey [WiggleZ Collaboration, 2012] on linear scales with the CosmoMC code [Lewis and Bridle, 2002] using nested sampling, as implemented in the MultiNest code [Feroz et al. $[2009]$. They conclude that within the large mass range $-32 \leq \log _{10}(\mathrm{~m} / \mathrm{eV}) \leq-25.5$ the FDM to total DM fraction can not exceed five percent at a $95 \%$-confidence. If CDM is entirely made of FDM, masses $m \leq 10^{-24} \mathrm{eV}$ are ruled out by observations. The analysis was refined by Hložek et al. [2018] using the full Planck data set. Including CMB lensing, constraints could be improved by up to a factor of two compared 
to using temperature anisotropies alone. The authors also investigate FDM isocurvature perturbations. Future CMB-S4 experiments will significantly improve the above limits Hložek et al., 2017, 2018]. Introducing a new set of physically more insightful quantities to evolve initial FDM density perturbations, Ureña-López and Gonzalez-Morales 2016 arrive at similar exclusions employing an amended version of the CMB code CLASS LLesgourgues, 2011]. Possible degeneracies with massive neutrinos have been investigated by Marsh et al. [2012] and Hložek et al. [2017]

Finally, the suppression of small-scale perturbations in FDM cosmologies alters the sub-horizon evolution of the Newtonian potential in the pre-recombination era. This in turn modifies spectral distortions in the CMB caused by Silk damping. Sarkar et al. [2017] estimate the deviations to be detectable by the upcoming PIXIE experiment Kogut et al. 2011.

\subsubsection{Halo Mass Function}

The halo mass function (HMF) quantifies the redshift dependent number density of DM halos $n(M, z)$ per logarithmic mass interval. From Press-Schechter theory, it follows that Press and Schechter, 1974

$$
\frac{d n}{d \ln M}=-\frac{\bar{\rho}}{M} f\left(\delta_{\text {crit }} / \sigma\right)\left|\frac{d \ln \sigma^{2}}{d \ln M}\right| .
$$

Extending the original analysis to ellipsoidal collapse, Sheth and Tormen 1999 found

$$
f(\nu)=A \sqrt{\frac{1}{2 \pi}} \sqrt{q} \nu\left[1+(\sqrt{q} \nu)^{-2 p}\right] \exp \left[-\frac{q \nu^{2}}{2}\right]
$$

with $A=0.3222, p=0.3$, and $q=0.707$. This semi-analytic result agrees reasonably well with CDM N-body simulations. Smoothing the density field by convolution with an appropriate window function $W(x \mid R(M))$ of characteristic radius $R$ corresponding to a mass $M \sim \bar{\rho} R^{3}$, the mass variance $S(M, z)$ is defined via the power spectrum $P(k, z)$ of the density perturbations:

$$
S(M, z)=\sigma^{2}(M, z)=\frac{1}{2 \pi} \int_{0}^{\infty} P(k, z) W^{2}(k \mid R(M)) k^{2} \mathrm{~d} k .
$$

Due to scale-independent growth, the critical overdensity of collapse is constant in an Einstein-de Sitter universe - appropriate for a standard $\Lambda$ CDM universe during structure formation - implying $\delta_{\text {crit,EdS }}(z) \simeq 1.686 D(0) / D(z)$. Here, $D(z)$ is the linear growth factor. For FDM, $\delta_{\text {crit }}(M, z)$ steeply rises for masses below $M\left(r_{J}(z)\right)$ as Jeans stability prevents overdensities to collapse on those scales Marsh, 2016a. Together with the suppression of small-scale density perturbations quantified by the FDM transfer function, this results in a sharp cutoff in the HMF below a minimum mass. Even if there were initial density perturbations below the Jeans scale they could not collapse into virialized objects. The 
Jeans scale $r_{J}$ is especially large for high redshifts, since in the early universe perturbations had less time to grow. For an FDM mass $m_{22} \equiv 10^{-22} \mathrm{eV}$ we have $M\left(r_{J}(z)\right) \sim 10^{9} M_{\odot}$ Marsh and Silk, 2013, Bozek et al., 2015]. Using the above halo model and a modified concentration-mass relationship for the halo density profiles, the non-linear power spectrum was semi-analytically constructed by Marsh 2016a].

Equation 2.40 is obtained by integrating Bond et al., 1991; Benson et al., 2012

$$
\int_{0}^{S} f\left(S^{\prime}\right) \operatorname{erfc}\left[\frac{B(S)-B\left(S^{\prime}\right)}{\sqrt{2\left(S-S^{\prime}\right)}}\right] \mathrm{d} S^{\prime}=\operatorname{erfc}\left[\frac{B(S)}{\sqrt{2 S}}\right]
$$

for

$$
B(S)=\delta_{\text {crit }, \operatorname{EdS}}(z) \sqrt{q}\left[1+b\left(\frac{1}{q \nu^{2}}\right)^{c}\right]
$$

with $b=0.5$ and $c=0.6$, appropriate for ellipsoidal collapse of CDM. Since the barrier $B$ deviates from Equation 2.43 for FDM, so does $f(S)$. Taking this into account results in an order one higher, less redshift-dependent cutoff [Du et al., 2016].

From numerical simulations, Schive et al. [2016] found that for $\left(m \sim 10^{-22} \mathrm{eV}, z \sim\right.$ $\left.4-10, M \gtrsim 10^{9} M_{\odot}\right)$ the FDM HMF can be well fitted by

$$
\left.\frac{d n}{d M}\right|_{\mathrm{FDM}}(M, z)=\left.\frac{d n}{d M}\right|_{\mathrm{CDM}}(M, z)\left[1+\left(\frac{M}{M_{0}}\right)\right]^{-2.2},
$$

where $M_{0}=1.6 \times 10^{10} m_{22}^{-4 / 3} M_{\odot}$ again marks the characteristic mass scale below which the HMF starts to drop noticeably. The FDM HMF fit in Equation 2.44 is redshift-independent since FDM physics entered the employed N-body simulations only via modified initial conditions. While the low-mass end of the HMF depends on the detailed FDM physics, $M_{0}$ should be only mildly redshift-dependent since it is mainly determined during the radiationdominated epoch [Hu et al., 2000]. While this is confirmed by semi-analytic constructions of the FDM HMF [Du et al., 2016], they imply a much steeper cutoff. The less-pronounced cutoff from Schive et al. [2016] might be a result of the N-body scheme employed which does not suppress small-scale growth. Indeed, first modified N-body simulations approximately accounting for coherence effects also during non-linear evolution suggest additional suppression on small scales [Zhang et al. 2018a]. Further dedicated FDM simulations are needed to confirm this result. A different parameterization was used by Corasaniti et al. 2017

$$
\left.\frac{d n}{d M}\right|_{\mathrm{FDM}}(M, z)=\left.\frac{d n}{d M}\right|_{\mathrm{CDM}}(M, z)\left[1-\exp ^{-\frac{M}{M_{0}}}\right]^{\gamma} 10^{\alpha+\beta \frac{M_{0}}{M}},
$$

where now $\alpha, \beta, \gamma$ and $M_{0}$ have been fitted to numerical simulations at various redshifts.

The suppression of small-scale structures formed from FDM delays galaxy formation relative to standard CDM for which structures start growing already at matter-radiation 
equality. Numerical simulations show that with FDM the first bound objects collapse at $z \simeq 13$ [Schive et al., 2014a], whereas under $\Lambda \mathrm{CDM}$ the first objects should form at $z \simeq 50$ Abel et al. 2001]. Currently, the oldest galaxy was observed at a redshift of $z \simeq 11.09$ Oesch et al. [2016], close to the FDM bound for $m_{22} \sim 1$.

Modifying the merger tree algorithm implemented in GALACTICUS [Cole et al., 2000; Benson, 2012, Du et al. 2016] were able to also construct subhalo mass functions of Milky Way-sized halos. They show a similar suppression on small scales. Unfortunately, most of the model assumptions needed for the semi-analytic approach have not yet been calibrated to FDM, as high-resolution large-scale FDM simulations are still missing.

\subsubsection{UV Luminosity Function}

Due to experimental improvements there are increasingly large data sets on galaxies all the way to redshifts of $z \sim 11$ [Oesch et al., 2016; Bouwens et al., 2015b]. The upcoming JWST experiment will significantly extend this catalogue also to higher redshifts Gardner et al., 2006]. The rest frame UVLF $\phi\left(M_{A B}\right)$ quantifies the number density per absolute magnitude $M_{A B}$ of star forming galaxies. The observed UVLF is well-fitted by a Schechter function [Schechter, 1976]:

$$
\phi(L)=\frac{\phi_{\star}}{L_{\star}}\left(\frac{L}{L_{\star}}\right)^{\alpha} \exp \left(-\frac{L}{L_{\star}}\right)
$$

with $M=-2.5 \log \left(L / \mathrm{erg} \mathrm{s}^{-1} \mathrm{~Hz}^{-1}\right)+51.6$ and best fit parameters Bouwens et al., 2015b

$$
\begin{aligned}
\phi_{\star} & =0.47 \times 10^{-0.27(z-6)-3} \mathrm{Mpc}^{-3} \\
M_{\star} & =-20.95+0.01(z-6) \\
\alpha & =-1.87-0.10(z-6)
\end{aligned}
$$

Similar parameter values were obtained by Kuhlen and Faucher-Giguère 2012. Assuming that each DM halo hosts a single galaxy whose luminosity is monotonically related to the halo mass $M_{h}\left(M_{A B}\right)$, the abundance matching technique assigns a galaxy of a given $M_{A B}$ to the corresponding mass $M_{h}$ by requiring

$$
\Phi\left(<M_{A B}, z\right)=n\left(>M_{h}, z\right) .
$$

Here, $\Phi$ is the cumulative galaxy luminosity function counting all bright galaxies below $M_{A B}$, which equates to the most massive DM halos in a specific DM scenario Vale and Ostriker, 2004. The above equality can only be enforced if the underlying DM model predicts enough low-mass halos to match the faint end of the observed luminosity function. It can therefore provide an upper bound on the allowed suppression of low-mass halos which in turn translated to a lower bound on the FDM mass. The thus obtained bound is model independent since no specific relation $M_{h}\left(M_{A B}\right)$ is assumed. Abundance matching to the CDM HMF, Schive et al. 2016] find $m_{22} \geq 1.5(2 \sigma)$, while matching to the FDM HMF given in Equation 2.44 yields $m_{22} \geq 0.9(2 \sigma)$. These bounds are relaxed if FDM contributes only 
a fraction of all DM [Bozek et al. 2015]. Updated analyses produced comparable bounds $m_{22} \geq 8.0(3 \sigma)$ Menci et al. [2017] and $m_{22} \geq 1.6(>2 \sigma)$ [Corasaniti et al., 2017.

The $M_{h}\left(M_{A B}\right)$ relation can additionally be used as a prediction of a given theory. This would require dedicated high resolution FDM simulations including baryonic physics which are not yet available. Generally, a suppression on small scales implies an enhanced star formation rate within small FDM halos $M_{h}<M_{0}$ compared to CDM halos Corasaniti et al., 2017.

A similar bound on the FDM mass $m_{22} \geq 1.2(2 \sigma)$ was obtained by Schive et al. [2016] using the conditional UVLF [Cooray and Milosavljević, 2005], which describes the probability density of halos with mass $M_{h}$ to host galaxies with luminosity $L$ :

$$
\phi_{c}\left(L \mid M_{h}, z\right)=\frac{1}{\sqrt{2 \pi} \ln (10) \Sigma L} \exp \left\{-\frac{\log \left[L / L_{c}\left(M_{h}, z\right)\right]^{2}}{2 \Sigma^{2}}\right\}
$$

with Bouwens et al., 2015b

$$
L_{c}\left(M_{h}, z\right)=L_{0} \frac{\left(M_{h} / M_{1}\right)^{p}}{1+\left(M_{h} / M_{1}\right)^{q}}\left(\frac{1+z}{4.8}\right)^{r} .
$$

The UVLF is then obtained by integrating

$$
\phi(L, z)=\int_{0}^{\infty} \phi_{c}\left(L \mid M_{h}, z\right) \frac{d n}{d M_{h}}\left(M_{h}, z\right) d M_{h}
$$

and finding appropriate parameters $\left(L_{0}, M_{1}, \Sigma, p, q, r\right)$ Schive et al., 2016; Bouwens et al. 2015b]. The thus predicted UVLF is well fitted by Shchive et al., 2016

$$
\phi_{\mathrm{FDM}}(L)=\phi(L)\left[1+\left(\frac{L}{L_{\mathrm{FDM}}}\right)^{\gamma}\right]^{\beta / \gamma}
$$

with best fit parameters

$$
\begin{aligned}
M_{\mathrm{FDM}} & =-17.6+4.5 \log \left(m_{22} / 0.8\right)-5.0 \log ((1+z) / 7) \\
\beta & =1.70+0.04(z-6) \\
\gamma & =-1.20 .
\end{aligned}
$$

The additional factor in Equation 2.54 parametrizes the suppression of the UVLF at the faint end due to FDM physics compared to the CDM UVLF $\phi(L)$. Constructing the conditional UVLF from the semi-analytically obtained HMF [Du et al., 2016] would potentially yield an even stronger bound on the FDM mass due to the steeper cutoff.

The conditional UVLF approach was also used by Corasaniti et al. [2017], who obtain a lower bound $m_{22} \geq 1.6(>2 \sigma)$. The analysis was done on an updated data sample for the observed UVLF, accounting for dust extinction corrections that shift the UVLF to smaller magnitudes. Additionally, $L_{c}\left(M_{h}, z\right)$ was modelled as the ensemble average of the 
luminosity at fixed halo mass and redshift. This reduces the free parameter space to $\Sigma$ and a redshift dependent overall amplitude of the ensemble averaged luminosity.

The small-scale suppression of the UVLF can be best constrained with multiply lensed high redshift galaxies which are typically magnified by an order of magnitude, decreasing the limiting intrinsic luminosity down to $M_{A B} \sim-15$ in the Hubble Frontier Field Lam et al., 2014] and indicating vigorous star formation in halos with $M_{h} \sim 10^{9} M_{\odot}$ [Finlator et al., 2016]. The gravitational lensing bias $N_{\text {lensed }}(>L) / N_{\text {unlensed }}(>L)$ modifies the number density of high-redshift galaxies above a limiting luminosity Broadhurst et al. 1995

$$
N_{\text {lensed }}(>L)=(1 / \mu) N_{\text {unlensed }}(>L / \mu),
$$

where $\mu$ is the magnification factor. Due to the difference in sign in the faint-end slope of the UVLF, the bias for CDM enhances the number of faint high-redshift galaxies, while for FDM the turnover in the UVLF leads to fewer galaxies magnified above the flux limit [Schive et al. 2016]. This turnover has indeed been found by investigating the Hubble Frontier Field (HFF) data, suggesting a best fit FDM mass $0.8<m_{22}<3.2$ [Leung et al. 2018]. JWST has the potential to probe even fainter lensed galaxies [Mason et al., 2015 especially employing the same deep lenses as the HFF for which magnification maps are best understood [Lam et al., 2014, Diego et al., 2015].

\subsubsection{Reionization History}

The reionization history - the time evolution of the volume filling fraction of ionized hydrogen $Q_{\mathrm{HII}}(z)$ - balances the ionization of the neutral intergalactic medium (IGM) with the recombination of free electrons and protons [Kuhlen and Faucher-Giguère, 2012, Madau et al., 1999; Robertson et al., 2013; Schultz et al., 2014

$$
\frac{d Q_{\mathrm{HII}}}{d t}=\frac{\dot{n}_{\text {ion }}}{\bar{n}_{\mathrm{H}}}-\frac{Q_{\mathrm{HII}}}{\bar{t}_{\mathrm{rec}}},
$$

where $\bar{n}_{\mathrm{H}}$ is the mean comoving hydrogen number density, $\dot{n}_{\text {ion }}$ is the comoving production rate of ionizing photons per unit volume and

$$
\bar{t}_{\text {rec }}=\frac{1}{C_{\mathrm{HII}} \alpha_{B}\left(T_{0}\right) \bar{n}_{\mathrm{H}}(1+Y / 4 X)(1+z)^{3}} \sim 0.93\left(\frac{7}{1+z}\right)^{3} \mathrm{GeV}
$$

is the volume averaged recombination time of ionized hydrogen. The clumping factor of ionized gas $C_{\mathrm{HII}}=\left\langle n_{\mathrm{H}}^{2}\right\rangle /\left\langle n_{\mathrm{H}}\right\rangle^{2}=2 \sim 5$ varies based on definition and method [Robertson et al. 2013, but is typically chosen to be $C_{\mathrm{HII}}=3$ Kuhlen and Faucher-Giguère, 2012, Schultz et al., 2014, Robertson et al., 2015]. $\alpha_{B}$ is the case $B$ hydrogen recombination coefficient depending on the IGM temperature, commonly assumed to be $T_{0} \sim 2 \times 10^{4} \mathrm{~K}$, which is appropriate for ionized gas at the mean density during the epoch of reionization Hui and Haiman, 2003. The primordial hydrogen and helium mass fractions are well modeled by $X=0.76$ and $Y=0.24$ Kuhlen and Faucher-Giguère, 2012; Schultz et al. 
2014], consistent with both CMB measurements [Planck Collaboration, 2018] and estimates from low-metallicity extragalactic regions [Izotov and Thuan, 2004; Steigman, 2007]. The production rate of ionizing photons

$$
\dot{n}_{\text {ion }}=\frac{2 \times 10^{25}}{\operatorname{erg~} \mathrm{Hz}^{-1}} \zeta_{\text {ion }} f_{\text {esc }} \int_{L_{\text {lim }}}^{\infty} \phi(L) L \mathrm{~d} L
$$

depends on the UVLF $\phi(L)$ defined in Equation 2.54, on the efficiency of converting galaxy UV luminosity to ionizing photon luminosity $\zeta_{\text {ion }}=0.5 \sim 2.0$, on their escape fraction $f_{\text {esc }}=0.1 \sim 0.5$, and on the limiting UV magnitude $M_{\text {lim }}=-17 \sim-10$ Bozek et al. 2015; Schive et al., 2016; Kuhlen and Faucher-Giguère, 2012; Schultz et al., 2014; Bouwens et al. 2015a.

Energetic photons from early forming stars fully reionize the IGM by $z \sim 6$ with $Q_{\mathrm{HII}}(z \sim 6)>0.99$ Madau et al., 1999; Loeb and Barkana, 2001; Bunker et al., 2004; Yan and Windhorst, 2004; Oesch et al., 2009]. This can be inferred from observations of the Gunn-Peterson trough [Gunn and Peterson, 1965] in quasar spectra at $z>6$ Fan et al., 2006; Schroeder et al., 2012; McGreer et al., 2014; Dijkstra, 2014] and Lyman$\alpha$ forest measurements at $z<6$ [Becker et al., 2001; Djorgovski et al., 2001]. A more conservative constraint is obtained using the covering fraction of "dark" pixels in quasar spectra implying $Q_{\mathrm{HII}}(z=5.5)>0.8$ and $Q_{\mathrm{HII}}(z=6)>0.5$ [McGreer et al., 2011]. Other sources of reionization were found to be sub-dominant Haiman and Loeb, 1998; Fontanot et al., 2012. The observed reionization history suggests a significant ionizing photon escape fraction and a UVLF extending beyond the observed intrinsic luminosity and redshift Kuhlen and Faucher-Giguère, 2012; Robertson et al., 2013]. Since FDM delays structure formation, reionization starts at smaller redshifts compared to CDM. Reionization must thus be more efficient in FDM scenarios. Even for $\zeta_{\text {ion }} f_{\text {esc }} \sim 0.6$ as opposed to the standard $\mathrm{CDM}$ value $\zeta_{\text {ion }} f_{\text {esc }} \sim 0.2$, the FDM mass is constrained to $m_{22} \geq 0.73$ [Schive et al., 2016]. This bound can be relaxed if FDM constitutes only a fraction of DM [Bozek et al., 2015. . Recent measurements suggest even higher values $\zeta_{\text {ion }} f_{\text {esc }} \sim 1.0$ for faint high-redshift galaxies [Meyer et al., 2018; Dijkstra et al., 2016]. Additionally, an investigation of the Hubble Frontier Field suggests an enhanced star formation rate at redshifts $z \sim 8-10$ Leung et al., 2018]. Reionization can also suppress galaxy formation with observable imprints in the luminosity function of galaxies Bose et al., 2018].

Using an N-body scheme with FDM initial conditions to simulate the DM distribution at $z=8$, Sarkar et al. [2016] confirm the FDM HMF found by Schive et al. [2016] expect for a sudden increase for very small halo masses. It can be attributed to the non-subtraction of spurious halos appearing as numerical artifacts. This weakens the derived FDM mass constraints. They continue their analysis by semi-analytically generating the ionization map and neutral hydrogen (HI) distribution employing a homogeneous recombination scheme Choudhury et al., 2009|. It assumes that hydrogen exactly traces the matter density distribution and ionizing photons are only produced within halos in proportion to the halo mass. The ionization map is then constructed by comparing the smoothed photon number density to the smoothed hydrogen number density. Regions with higher photon than hydrogen number density are counted as ionized. The remaining HI distribution is finally 
mapped to redshift space Bharadwaj and Ali, 2004, Majumdar et al., 2013 in order to calculate the brightness temperature fluctuation [Bharadwaj and Ali. 2005]

$$
\delta T_{b}=4 \mathrm{mK} \frac{\rho_{\mathrm{HI}}}{\bar{\rho}_{\mathrm{H}}}(1+z)^{2}\left(\frac{\Omega_{b} h^{2}}{0.02}\right)\left(\frac{0.7}{h}\right) \frac{H_{0}}{H_{z}},
$$

where $\rho_{\mathrm{HI}} / \bar{\rho}_{\mathrm{H}}$ is the ratio of HI to the mean hydrogen density. Since FDM simulations show fewer halos, the remaining ones have to produce ionizing photons more efficiently in order to reionize the universe similarly to CDM scenarios. For $m_{22}<0.26$ no halos form excluding this mass range. In simulations with slightly higher masses, the needed photon production efficiency was still unrealistically high. However, large uncertainties prohibit conclusions on FDM mass constraints. Since FDM predicts fewer but brighter ionizing sources during the epoch of reionization, JWST will be able to distinguish between FDM and CDM by directly detecting them [Gardner et al., 2006].

The suppression of small halos results in larger ionized regions with stronger contrast compared to CDM simulations. This alters the mean squared brightness temperature fluctuation $\Delta_{b}^{2}(k)=k^{3} P_{b}(k) / 2 \pi^{2}$ of the HI field for FDM, which is enhanced over a wide range of scales $0.1<k<4 \mathrm{Mpc}^{-1}$ [Sarkar et al. 2016]. Generally, a suppression in the matter power spectrum implies an enhancement in $\Delta_{b}^{2}(k)$ [Furlanetto et al., 2004; Lidz et al. 2008). The HI signal is measured by various experiments including LOFAR (Hörandel et al. 2009], MWA [Webster, 2017], PAPER [Pober et al., 2015], but data does not yet have the required precision for FDM mass constraints.

An additional constraint on the FDM mass can be derived from damped Lyman- $\alpha$ absorption data Noterdaeme et al., 2009; Péroux et al., 2003; Noterdaeme et al., 2012; Zafar et al., 2013; Crighton et al., 2015; Songaila and Cowie, 2010]. Simulations indicate damped- $\alpha$ clouds in the mass range $10^{9}-10^{10} M_{\odot}$ [Pontzen et al., 2008]. Observations at $z \simeq 2.5$ suggest even more massive clouds up to $10^{12} M_{\odot}$ [Font-Ribera et al. 2012]. A lower bound on the collapsed fraction of FDM can be obtained from their combined observed mass that can be compared to the fraction of FDM mass within the heaviest FDM halos. Integrating the HMF above a limiting mass of $\sim 10^{10} M_{\odot}$, Sarkar et al. 2016 rule out FDM with mass $m_{22}<0.1$.

The reionization history can be best constrained by the CMB Thomson scattering optical depth, which is an integral over the full reionization history

$$
\tau=c \sigma_{\mathrm{T}} \bar{n}_{\mathrm{H}} \int_{0}^{\infty} \frac{(1+z)^{2}}{H(z)} Q_{\mathrm{HII}}(z)(1+\eta(z) Y / 4 X) \mathrm{d} z .
$$

Here, $c$ is the speed of light, $H(z)$ is the Hubble parameter, $\sigma_{\mathrm{T}}$ is the Thomson scattering cross-section, and $\eta(z)$ represents the ionization state of helium. It is typically taken to be $\eta(z>4)=1$ for singly and $\eta(z \leq 4)=2$ for doubly ionized helium Bozek et al., 2015, Schive et al., 2016; Kuhlen and Faucher-Giguère, 2012]. The optical depth was observed to be $\tau=0.054 \pm 0.007$ [Planck Collaboration, 2018]. Since previous data yielded larger values for $\tau$, the FDM mass limits obtained by Schive et al. [2016] and Bozek et al. 2015] are slightly too tight but suggest a rough limit of $m_{22} \geq 0.5$ compatible with above presented limits. 
Small-scale polarization data from CMB experiments like the Atacama Cosmology Telescope [ACT Collaboration, 2014] and South Pole Telescope [George et al., 2015] combined with Planck data [Planck Collaboration, 2016b] can accurately measure the kinematic Sunyaev-Zel'dovich (kSZ) effect. The kSZ power spectrum amplitude constrains the time $z_{\mathrm{re}}=z\left(Q_{\mathrm{HII}}=0.5\right)$ of reionization to be between $z=7.8$ and 8.8 and its duration $\delta z_{\mathrm{re}}=z\left(Q_{\mathrm{HII}}=0.75\right)-z\left(Q_{\mathrm{HII}}=0.25\right)<2.8$ [Planck Collaboration, 2016a]. Together with the small value for $\tau$ this suggests a delayed, more efficient reionization then previously assumed. These measurements could distinguish between CDM and FDM even for larger FDM masses $m_{22} \sim 10$ Bozek et al., 2015]. Unfortunately, an updated analysis including the newest Planck data [Planck Collaboration, 2018] has not been done yet.

\subsubsection{Lyman-Alpha Forest}

The Lyman- $\alpha(\operatorname{Ly} \alpha)$ forest is a set of absorption lines in high-redshift quasar spectra. While propagating to earth the quasar photons are redshifted to larger wavelength. Photons passing the Ly $\alpha$ resonance at a wavelength of $\lambda \simeq 1216 \AA$ are efficiently absorbed by HI clouds at the corresponding redshift. The observed absorption lines blue-ward the (redshifted) Ly $\alpha$ resonance thus directly trace the HI distribution along the line-of-sight quantified by the one dimensional matter power spectrum

$$
P_{1 D}(k)=\frac{1}{2 \pi} \int_{k}^{\infty} k^{\prime} P_{3 D}\left(k^{\prime}\right) \mathrm{d} k^{\prime} .
$$

If sufficiently resolved, the forest can be modelled by fitting each individual line with a Voigt profile using $\chi^{2}$ minimization [Mo et al. [2010]. Their depth measures the column density while their width quantifies the temperature of the individual HI cloud.

The Ly $\alpha$ forest has already been successfully used to constrain the warm DM (WDM) mass $m_{\mathrm{WDM}} \gtrsim 4.09 \mathrm{keV}$ Baur et al., 2016], which suppresses DM density perturbations below a Jeans scale set by free-streaming. Relating the Jeans scales for WDM at $T \sim$ $m_{\mathrm{WDM}}$ and FDM at $H(T) \sim m_{\mathrm{FDM}}$ during radiation domination Marsh and Silk, 2013; Marsh, 2016b

$$
\frac{m_{\mathrm{WDM}}}{\mathrm{keV}} \simeq \frac{\sqrt{m_{\mathrm{FDM}} M_{\mathrm{Pl}}}}{\mathrm{keV}} \simeq 0.5\left(\frac{m_{\mathrm{FDM}}}{10^{-22} \mathrm{eV}}\right)^{0.5}
$$

it can be expected that Ly $\alpha$ forest measurements can constrain FDM masses $m_{22} \gtrsim 70$. Early Ly $\alpha$ analyses indeed disfavor $m_{22}<1$ [Amendola and Barbieri, 2006]. Using hydrodynamical simulations including star formation in cold and dense baryon environments [Bolton et al., 2016] with FDM initial conditions and comparing them to high-statistics Ly $\alpha$ power spectra measured by the BOSS survey [Palanque-Delabrouille et al., 2013], VLT [López et al., 2016] and spectra summarized in Viel et al. [2013], Armengaud et al. [2017] find $m_{22} \gtrsim 29$, while Iršič et al. [2017] obtain $m_{22} \gtrsim 20$ and $m_{22} \gtrsim 37.5$ depending on the assumed thermal history. In both simulations, the redshift-dependent IGM temperature is modelled by

$$
T_{\mathrm{IGM}}=T_{0}(z)(1+\delta \rho / \rho)^{\gamma(z)-1},
$$


while the effective optical depth is given by

$$
\tau_{\text {eff }}=\alpha \times(1+z)^{\beta} .
$$

The parameters were set to $T_{0}(z=3)=1400 \mathrm{~K}$ and $\gamma(z=3)=1.3$ in agreement with Becker et al. [2010] subject to a power-law redshift dependence and $\alpha=0.0025$ and $\beta=3.7$ in Armengaud et al. [2017] and similar values in Iršič et al. [2017]. The above analysis was refined by Kobayashi et al. [2017], investigating also scenarios in which FDM constitutes only a fraction of the total DM energy density.

The results indicate FDM might suffer from the same catch -22 problem as WDM. This tension could be alleviated if the axion is initially near its potential maximum Zhang and Chiueh, 2017bal. Due to parametric instabilities in this extremal misalignment case, the power spectrum at high redshifts $z \sim 100$ is enhanced above the Jeans scale with respect to its CDM and standard FDM counterparts SSchive and Chiueh, 2017; Cedeño et al., 2017]. A caveat in the employed simulations is the use of an N-body scheme as implemented in Gadget-3 [Springel, 2005] in order to model the time evolution of FDM. The small-scale coherence effects of FDM are therefore neglected. An a posteriori analysis of the force due to coherence effects verses the gravitational force suggests that a full FDM treatment would produce similar bounds [Armengaud et al., 2017]. However, the granular structure and solitonic cores in filaments and halos found in full FDM simulations Schive et al. 2014a could not be properly accounted for. Since dedicated simulations have proven that large scales are not affected by these non-linearities Veltmaat and Niemeyer, 2016, it is argued that especially the BOSS data is insensitive to these effects as only larger scales are probed. This is different for the high-resolution spectra additionally used. Constraints deduced from them can be expected to slightly change when using full FDM simulations. Indeed, a first numerical N-body study, in which coherence effects are approximately taken into account, suggests enhanced suppression of small-scale density perturbations Zhang et al. 2018a.

\subsubsection{EDGES and the $21 \mathrm{~cm}$ Line}

Since Ly $\alpha$ forest measurements rely on a limited number of high-redshift quasars, it is difficult to statistically quantify the structure of reionization, while the large optical depth of HI even prevents accurate measurements of the redshift-dependent ionization fraction at the beginning of reionization, and the observed optical depth of the CMB provides only redshift-integrated constraints.

These limitations can be overcome by measuring HI $21 \mathrm{~cm}$ lines that correspond to a spin-flip transition in the ground state of hydrogen. The details of $21 \mathrm{~cm}$ cosmology summarized here can be found in Mo et al. [2010] and Lidz and Hui [2018], while a more in depth discussion is presented in Furlanetto et al. [2006] and Pritchard and Loeb [2012]. Historically it was first investigated by Wouthuysen [1952], Field [1958], Field] [1959] and then Madau et al. [1997].

The spin-spin coupling of the proton and electron breaks the degeneracy of the ground state resulting in a hyperfine structure splitting depending on the parallel (triplet state 
$F=1$ ) or anti-parallel (singlet state $F=0$ ) spin alignment. Its energy difference, $E_{10}=$ $E_{1}-E_{0} \simeq 5.9 \times 10^{-6} \mathrm{eV}$, corresponds to a wavelength of $\lambda_{10} \simeq 21 \mathrm{~cm}$, a frequency $\nu_{10} \simeq 1.4$ $\mathrm{GHz}$, and a temperature $T_{10} \simeq 0.068 \mathrm{~K}$. In equilibrium, the relative abundance of atoms in the two different states is characterized by the spin temperature $T_{s}$ :

$$
\frac{n_{1}}{n_{0}}=3 \exp \left[-\frac{T_{10}}{T_{s}}\right],
$$

where the factor of three accounts for the degeneracy of the triplet $F=1$ state. Travelling through HI, photons with temperature $T_{\gamma}$ - for example from the CMB — can be absorbed by exciting hydrogen atoms from the singlet to the triplet state. The resulting change in brightness temperature in the limit of small optical depth $\tau \ll 1$ and high redshift $z \gg 1$

$$
\begin{aligned}
\delta T & =T_{b}-T_{\gamma}=\frac{\left(1-\exp ^{-\tau(z)}\right)\left[T_{s}(z)-T_{\gamma}(z)\right]}{(1+z)} \\
& \simeq 28 \mathrm{mK}\left(\frac{\Omega_{b, 0} h}{0.03}\right)\left(\frac{\Omega_{m, 0}}{0.3}\right)^{-1 / 2}\left(\frac{1+z}{10}\right)\left(1-\frac{T_{\gamma}}{T_{s}}\right)(1+\delta) x_{\mathrm{HI}}
\end{aligned}
$$

then depends on the neutral fraction $x_{\mathrm{HI}}$ and overdensity $\delta$. If the photons are in thermal equilibrium with the gas, $T_{\gamma}=T_{s}$, there is no net effect since absorption is exactly compensated by emission. Instead, $T_{\gamma}>T_{s}\left(T_{\gamma}<T_{s}\right)$ leads to net absorption (emission).

There are three known processes that change the ratio of atoms in the two hyperfine states and thus determine the spin temperature. The absorption/emission of radio background photons with temperature $T_{\gamma}$ - usually associated to the CMB - couples $T_{s}$ to $T_{\gamma} \simeq T_{s}$. Additionally, HI atoms can change their hyperfine state when colliding with each other or via the Wouthuysen-Field effect |Wouthuysen, 1952; Field, 1958] whereby a HI atom resonantly scatters off a Ly $\alpha$ photon and thus flips spin via an intermediate excited state. The last two processes couple the spin temperature to the kinetic temperature of the gas $T_{K}$ and the brightness temperature of the radiation field $T_{\alpha}$, respectively:

$$
T_{s}=\frac{T_{\gamma}+y_{\alpha} T_{\alpha}+y_{c} T_{K}}{1+y_{\alpha}+y_{c}},
$$

where

$$
y_{c}=\frac{C_{10}}{A_{10}} \frac{T_{10}}{T_{K}} \quad \text { and } \quad y_{\alpha}=\frac{P_{10}}{A_{10}} \frac{T_{10}}{T_{\alpha}} .
$$

Here, $C_{10}\left(T_{K}\right)$ is the rate of collisional de-excitation of the triplet state, $A_{10} \simeq 2.9 \times 10^{-15}$ $\mathrm{s}^{-1}$ is its spontaneous decay rate, and $P_{10}=4 P_{\alpha} / 27$ is the de-excitation rate due to the total Ly $\alpha$ photon scattering rate $P_{\alpha}$. At high redshift, Compton scattering between CMB photons and residual free electrons in the IGM drives $T_{K}$ towards $T_{\gamma} \simeq T_{K} \sim(1+z)$ when the energy density of the CMB and the ionization fraction in the hydrogen density are still large. Below $z \sim 200$ this process becomes inefficient and $T_{K} \sim(1+z)^{2}$ instead decreases like an adiabatically expanding non-relativistic gas. This reduces $T_{s}$ with respect to $T_{\gamma}$ as 
long as collisional (de-)excitation drives $T_{s}$ towards $T_{K}$. Around $z \sim 70$ the IGM became too dilute and cold for a sufficient collision rate and $T_{s}$ is again tightly coupled to $T_{\gamma}$ Furlanetto et al., 2006. This in turn makes the IGM essentially invisible by $z \sim 30$ until star formation produces Ly $\alpha$ photons that trigger the Wouthuysen-Field effect. Neglecting collisional (de-)excitation, (2.68) can then be rearranged to

$$
1-\frac{T_{\gamma}}{T_{s}}=\frac{y_{\alpha}}{1+y_{\alpha}}\left(1-\frac{T_{\gamma}}{T_{K}}\right),
$$

assuming that the large cross section of HI near the Ly $\alpha$ resonance and the photons' ability to exchange energy with the gas through the recoil of atoms while emitting Ly $\alpha$ photons, thermalizes the radiation spectrum near Ly $\alpha$ with the gas, which drives $T_{\alpha}$ quickly to $T_{K}$ [Field, 1959].

The observed negative brightness temperature between $z \sim 20$ and $z \sim 15$ by the EDGES experiment Bowman et al., 2018 thus places the onset of star formation around $z \sim 20\left(y_{\alpha}(z<20)>0\right)$. At this redshift, the gas temperature is expected to be 9.3 $\mathrm{K}$, further dropping to $5.4 \mathrm{~K}$ at $z=15$ [Bowman et al., 2018]. Since the CMB temperature $T_{0}(1+z)$ with $T_{0}=2.725$ scales linearly with redshift the corresponding radiation background temperature is expected to be $T_{\gamma} \simeq 57.2 \mathrm{~K}$ and $T_{\gamma} \simeq 43.6 \mathrm{~K}$, respectively. Thus, even for the most extreme case, in which $T_{s}$ is fully coupled to $T_{K}\left(y_{\alpha} \gg 1\right)$, the implied maximum absorption amplitude is $0.2 \mathrm{~K}$ at $z=20$ and $0.23 \mathrm{~K}$ at $z=15$, in clear contradiction to the EDGES results showing a deficit of $\simeq 0.5 \mathrm{~K}$ Bowman et al., 2018]. This indicates an increased $T_{\gamma} \simeq 104 \mathrm{~K}$ or a decreased $T_{K} \simeq 3.2 \mathrm{~K}$ at $z \sim 17$. The tension further increases when matter perturbations are taken into account [Xu et al., 2018]. Eventually, Ly $\alpha$ and X-ray photons heat the gas above the radiation temperature, ending the absorption signal. EDGES data suggests this transition to happen around $z \sim 15$ [Bowman et al., 2018]. It was found that these results are consistent with an extrapolation of the declining integrated UVLF measured at $4 \leq z \leq 9$ [Bouwens et al., 2015b], if the UVLF does not decline more rapidly for $z \gtrsim 10$ and the ionizing photon escape fraction is $f_{\text {esc }} \lesssim 0.2$ Madau, 2018. The reliability of the EDGES results is strongly debated. In fact, Hills et al. 2018 obtained a satisfactory fit to the data without any absorption feature, if there is a periodic feature with an amplitude of $\sim 0.05 \mathrm{~K}$ present in the data.

The redshift-dependent coupling coefficient $y_{\alpha}$ quantifies the Wouthuysen-Field effect and thus the star formation history in the early universe. It is given by Pritchard and Loeb, 2012; Lidz and Hui, 2018,

$$
y_{\alpha}=\frac{16 \pi^{2} T_{10} e^{2} f_{\alpha}}{27 A_{10} T_{\gamma} m_{e} c} S_{\alpha} J_{\alpha} \simeq \frac{J_{\alpha}(z) / J_{0}(z)}{0.069}\left(\frac{1+z}{21}\right)^{2},
$$

where Chen and Miralda-Escude, 2004

$$
J_{0}=\frac{c n_{H}(z)}{4 \pi \nu_{\alpha}}
$$

is the specific intensity equivalent to one Ly $\alpha$ photon per hydrogen atom. In order to achieve the fiducial value $y_{\alpha}=1$ at $z=20$ suggested by the EDGES results therefore 
requires a specific intensity of 0.069 Ly $\alpha$ photons per hydrogen atom [Lidz and Hui, 2018]. It can be calculated according to Hirata, 2006; Furlanetto, 2006

$$
J_{\alpha}(z)=\frac{c}{4 \pi}(1+z)^{2} \sum_{n=2}^{n_{\max }} f_{\text {recycle }}(n) \int_{z}^{z_{\max }(n)} \frac{\epsilon\left(\nu, z^{\prime}\right)}{H\left(z^{\prime}\right)} \mathrm{d} z^{\prime}
$$

where

$$
z_{\max }(n)=(1+z) \frac{1-(n+1)^{-2}}{1-n^{-2}}-1
$$

and the sum over $n$ runs over Lyman-series resonances and $f_{\text {recycle }}(n)$ quantifies the creation of Ly $\alpha$ photons due to de-excitation cascades from the $n$th energy level. The coefficients $f_{\text {recycle }}(n)$ were calculated by Pritchard and Furlanetto [2006], and $n_{\text {max }}=23$ is suggested in Furlanetto [2006]. Assuming that the UV emissivity traces the fraction of DM collapsed into halos of sufficient minimal mass $M_{\min }$ to trigger star formation, it is approximated by Furlanetto, 2006

$$
\epsilon\left(\nu, z^{\prime}\right)=\epsilon(\nu) f_{\star} n_{H}(z=0) \frac{d f_{\mathrm{coll}}\left(>M_{\min }, z^{\prime}\right)}{d t},
$$

where $f_{\star}$ is the star formation efficiency and $\epsilon(\nu)=N_{\alpha} \nu^{-\alpha_{s}-1}$ is the specific emissivity with fiducial parameters $N_{\alpha}=9690$ and $\alpha_{s}=-0.14$ [Barkana and Loeb, 2005].

Employing the above analysis, Schneider 2018] and Lidz and Hui [2018] obtain a lower bound on the FDM mass by using the above described FDM HMFs of [Schive et al. [2016], Marsh and Silk [2013] and Marsh [2016a]. In their analysis Lidz and Hui [2018] vary $M_{\text {min }}=10^{6}-10^{9} M_{\odot}$, but find no strong dependence of the specific value due to the low mass suppression in the FDM HMF. Assuming $f_{\star}=0.05$, Lidz and Hui [2018] conclude that only FDM masses $m_{22}>50$ can produce $y_{\alpha}>1$ at $z=20$. The lower star formation efficiency $f_{\star}=0.03$ considered by Schneider 2018] increases this bound to $m_{22}>80$.

\subsubsection{Pulsar Timing Arrays}

We have already shown that the temporal part of the energy momentum tensor of the scalar FDM field given in Equation 2.9 reduces to a constant density equal to the absolute square of the wavefunctions amplitude in Equation 2.14. On the contrary, for $H \ll m$, the dominant term in the spatial components oscillate in time Khmelnitsky and Rubakov, 2014:

$$
T_{i j}=-\rho \cos (2 m t+2 \alpha(x)) \delta_{i j}=p(x, t) \delta_{i j} .
$$

Since the momentum $p(x, t)$ oscillates harmonically around zero on the Compton time scale, averaging over one period results in a negligible pressure and an average equation of state $\omega=0$ as required for DM and discussed below Equation 2.38. However, as shown by Khmelnitsky and Rubakov [2014], the oscillating pressure induces oscillations in the 
gravitational potential, which can lead to observable effects in pulsar timing experiments. In this section we closely follow their derivation as presented by Khmelnitsky and Rubakov 2014.

Using Equation 2.7, the trace of spatial components of the Einstein Equation 2.5 yields

$$
-6 \partial_{t}^{2} U+2 \nabla^{2}(U-V)=8 \pi G T_{k k}=24 \pi G p .
$$

Decomposing the potential

$$
V(x, t) \simeq V_{0}(x)+V_{c} \cos (2 m t+2 \alpha(x))+V_{s} \sin (2 m t+2 \alpha(x))
$$

into a time-independent component $V_{0}$ and oscillating parts proportional to $V_{c}$ and $V_{s}$, and similarly for $U(x, t)$, it follows that $V_{0}=U_{0}$, obeying the usual Poisson Equation 2.8. Thus, $V_{0} \sim G \rho k^{-2}$ is quadratically suppressed by the wave number $k$. Neglecting spatial derivatives, the time-dependent parts are proportional to

$$
V_{s}(x)=0 \quad V_{c}(x)=G \pi \rho(x) m^{-2} .
$$

In the non-relativistic regime, the oscillations are therefore small perturbations with relative amplitude $V_{c} / V_{0} \sim k^{2} / m^{2}=v^{2} \sim 10^{-6}$. Similar to the Sachs-Wolf effect for CMB photons discussed above, the time variations in the metric induce frequency shifts $\left(\Omega\left(t^{\prime}\right)-\Omega_{0}\right)$ and a corresponding time delay

$$
\Delta t(t)=-\int_{0}^{t} \frac{\Omega\left(t^{\prime}\right)-\Omega_{0}}{\Omega_{0}} \mathrm{~d} t^{\prime}
$$

for any signal with pulse emission frequency $\Omega_{0}$ at the pulsar. It can show up as a residual variation of an arriving signal in pulsar timing array experiments. The integrand is given by

$$
\frac{\Omega(t)-\Omega_{0}}{\Omega_{0}}=V(x, t)-V\left(x_{0}, t_{0}\right)-\int_{t_{0}}^{t} n_{i} \partial_{i}\left(V\left(x^{\prime}, t^{\prime}\right)+U\left(x^{\prime}, t^{\prime}\right)\right) \mathrm{d} t^{\prime},
$$

where $n_{i}$ is the unit vector in the direction of the signal propagation and the integral is taken over its unperturbed trajectory. The distance to observed pulsars is typically $D \gtrsim 100 \mathrm{pc} \gg m^{-1}$. The integrand in Equation 2.81 is thus highly oscillatory, suppressed by $k / m=v \sim 10^{-3}$, and can therefore safely be neglected. Then,

$$
\Delta t(t)=\frac{V_{c}}{m} \sin \left(m D+\alpha(x)-\alpha\left(x_{0}\right)\right) \cos \left(2 m t+\alpha(x)+\alpha\left(x_{0}-m D\right)\right),
$$

where now $t$ denotes the signal propagation time from the pulsar at $x_{0}$ to the observer at $x$ separated by a distance $D$. Averaging the square of the amplitude in Equation 2.82 over the distance $D$ yields

$$
\sqrt{\left\langle\Delta t_{\mathrm{FDM}}^{2}\right\rangle}=\sqrt{2} \frac{V_{c}(x)}{\omega}
$$


with angular frequency $\omega=2 \mathrm{~m}$. This result can be compared to gravitational wave signals

$$
\sqrt{\left\langle\Delta t_{\mathrm{GW}}\right\rangle}=\frac{1}{\sqrt{3}} \frac{h_{c}}{\omega}
$$

with characteristic strain

$$
h_{c}=\sqrt{6} V_{c}=2 \times 10^{-17}\left(\frac{\rho}{0.3 \mathrm{GeV} / \mathrm{cm}^{3}}\right) m_{22}^{-2}
$$

at frequency

$$
f=2 \pi \omega=5 \times 10^{-8} m_{22} \mathrm{~Hz} .
$$

Khmelnitsky and Rubakov 2014] conclude that the SKA experiment is potentially sensitive enough to detect an FDM signal if $m_{22} \sim 0.1$. Since it is monochromatic, it can be distinguished from a stochastic gravitational wave background. Employing a Bayesian analysis, it is shown by Porayko and Postnov [2014], that the best current limits obtained by the NANOGrav PTA experiment [NANOGrav Collaboration, 2018] are an order of magnitude above the required sensitivity even for this best constrainable FDM mass range $m_{22} \sim 0.1$.

It was emphasized by Martino et al. [2017], that the above analysis assumes a constant density background. However, many observable pulsars can be expected to lie in the solitonic core region of the Milky Way. Since the strain as defined in Equation 2.85 is proportional to the local FDM density, the signal can be enhanced by up to three orders of magnitude, such that even current experiments start to be able to constrain the parameter space around $m_{22} \sim 0.1$. Additionally, the strong density contrast on de Broglie length scales in the granular density profile of FDM halos found in cosmological simulation Schive et al. 2014a could potentially be detected by investigating residual time modulations between individual pairs of pulsars.

\subsubsection{Gravitational Lensing}

From general relativity it is known that light is bent around massive objects, that can therefore serve as gravitational lenses [Bartelmann and Schneider, 2001; Kilbinger, 2018]. Quantifying this effect, we follow Mo et al. [2010] and start defining the photon trajectories under the influence of a Newtonian potential $V$. Employing the line element from Equation 2.7 in comoving coordinates

$$
\mathrm{d} s^{2}=a^{2}(\tau)\left[(1+2 V) \mathrm{d} \tau^{2}-(1-2 V)\left(\mathrm{d} \chi^{2}+f_{K}^{2}(\chi) \mathrm{d} \Omega^{2}\right)\right]
$$

they are given via the corresponding geodesic equation

$$
\frac{d \hat{u}}{d \chi}=-2 \nabla_{\perp} V
$$


where $u=d x / d \tau$ represents the direction of the light propagation and $\nabla_{\perp}$ denotes the gradient perpendicular to the unit vector $\hat{u}$. The right hand side quantifies the photon deflection $\delta \alpha_{d}=-2 \nabla_{\perp} V \delta \chi$. Considering a source at $\chi_{S}\left(x_{\perp, S}\right)$ in a perturbed (unperturbed) universe, its image position at the observer's position $x_{\perp, 0}$ is thus deflected by

$$
x_{\perp, 0}=x_{\perp, S}-2 \int_{\chi_{S}}^{0} f_{K}\left(\chi_{S}-\chi\right) \nabla_{\perp} V(\chi) \mathrm{d} \chi,
$$

proportional to the angular diameter distance $f_{K}\left(\chi_{S}-\chi\right)$ in comoving coordinates. Reformulating the above equation in angular positions of the source $\theta_{S}=x_{\perp, S} f_{K}\left(\chi_{S}\right)^{-1}$ and image $\theta_{0}=x_{\perp, 0} f_{K}\left(\chi_{S}\right)^{-1}$ relative to that of the lens, we obtain the lensing equation

$$
\theta_{S}=\theta_{0}-2 \int_{0}^{\chi_{S}} \frac{f_{K}\left(\chi_{S}-\chi\right)}{f_{K}\left(\chi_{S}\right)} \nabla_{\perp} V(\chi) \mathrm{d} \chi .
$$

In typical astrophysical applications significant lensing happens only in a small region around the gravitational lens much smaller than the lens-to-source or the observer-to-lens distance. Equation 2.90 can then be approximated by

$$
\theta_{S} \simeq \theta_{0}-2 \frac{f_{K}\left(\chi_{S}-\chi_{L}\right)}{f_{K}\left(\chi_{S}\right)} \int_{0}^{\chi_{S}} \nabla_{\perp} V(\chi) \mathrm{d} \chi .
$$

If we further assume a thin spherically symmetric lens like an NFW halo with solitonic core, the deflection angle

$$
\alpha_{d}=2 \int_{0}^{\chi_{S}} \nabla_{\perp} V(\chi) \mathrm{d} \chi=\frac{4 G M(\xi)}{\xi} \quad \text { where } \quad M(\xi)=2 \pi \int_{0}^{\xi} \hat{\xi} \Sigma(\hat{\xi}) \mathrm{d} \hat{\xi}
$$

becomes a function of the projected surface mass density

$$
\Sigma(\xi)=\int \rho(\xi, z) \mathrm{d} z .
$$

Here, $z$ is the distance to the lens projected along the photon trajectory and $\xi$ denotes the distance from the symmetry point of the lens in the lens plane. Defining the critical surface density

$$
\Sigma_{\mathrm{cr}}=\frac{d_{S}}{4 \pi G d_{L} d_{L S}}
$$

where $d_{i}=f_{K}\left(\Delta \chi_{i}\right)\left(1+z_{i}\right)^{-1}$ are angular diameter distances in physical coordinates, the lens Equation 2.90 can be rewritten as

$$
\theta_{S} \simeq \theta_{0}-\frac{M\left(\theta_{L}\right)}{\pi \Sigma_{\mathrm{cr}} d_{L}^{2} \theta_{L}},
$$


with $\xi=d_{l} \theta_{L}$. By definition, at the Einstein radius $\theta_{E}$, the mean surface density

$$
\bar{\Sigma}(\xi)=\frac{M(\xi)}{\pi \xi^{2}}
$$

within $\xi$ is equal to $\Sigma_{\text {cr }}$ and therefore $\theta_{S}=0$.

Gravitational lensing from FDM halos has been investigated by Herrera-Martín et al. 2017 in order to constrain the FDM profile given in Equation 2.83 below and thereby also the FDM mass. As the profile $\rho(r)$ in Equation 2.83 is assumed to be universal for all FDM halos with characteristic density $\rho_{s}$ and radius $r_{s}$, the above equations can be rewritten in dimensionless quantities relative to these characteristic scales with a single scale-dependent parameter

$$
\lambda=\frac{\rho_{s} r_{s}}{\pi \Sigma_{\mathrm{cr}}}=\frac{\theta_{E}^{\star 2}}{M^{\star}\left(\theta_{E}^{\star}\right)}=10^{-3} \frac{0.57}{h}\left(\frac{\rho_{s} r_{s}}{M_{\odot} \mathrm{pc}^{-2}}\right) \frac{d_{L} d_{L S}}{d_{S}} .
$$

Denoting dimensionless quantities with an asterisk the lens Equation 2.90 becomes

$$
\theta_{S}^{\star}=\theta_{0}^{\star}-\lambda \frac{M^{\star}\left(\theta^{\star}\right)}{\theta^{\star}} .
$$

There is a critical value

$$
\lambda_{\text {cr }}^{-1}=\pi \Sigma^{\star}(0)=2 \pi \int \rho^{\star}\left(r^{\star}\right) \mathrm{d} r^{\star}
$$

below which no Einstein ring can appear. It was found that $\lambda_{\mathrm{cr}, \mathrm{NFW}}=0$ for an NFW profile and $\lambda_{\mathrm{cr}, \mathrm{sol}} \simeq 0.5$ for a solitonic profile Herrera-Martín et al., 2017]. From Equation 2.97 we see, that while an NFW profile always allows for an Einstein ring, the solitonic profile can only produce Einstein rings if

$$
2.4 \times 10^{12}\left(\frac{r_{s}}{\mathrm{pc}}\right)^{-3} m_{22}^{-2}=\frac{\rho_{s} r_{s}}{M_{\odot} \mathrm{pc}^{-2}} \geq 10^{3} \frac{h}{0.57} \frac{d_{S}}{d_{L} d_{L S}} \lambda_{\mathrm{cr}} .
$$

Unfortunately, the general profile given in Equation 2.83 has too many free parameters in order to produce a stringent bound on the FDM mass. Using SLACS data Auger et al., 2009] for several lens candidates with strong lensing, Herrera-Martín et al. 2017. conclude that reasonable FDM halo profiles compatible with the data can be obtained if $m_{22}>0.01$. Employing the universal profiles numerically found by Mocz et al. [2017] together with the core-halo mass relation given in Equation 3.8 could further constrain the investigated system and potentially produce stronger bounds on the FDM mass.

FDM cosmologies can potentially also be constrained by measurements of weak gravitational lensing with statistically distributed galaxies Marsh et al., 2012]. For this investigation the lensing Equation 2.90, describing the deflection of a single photon trajectory has to be extended to infinitesimal regions around these beams. They are characterized by the Jacobian matrix [Mo et al., 2010]

$$
A_{i j}\left(\theta_{0}, \chi_{S}\right)=\frac{\partial \theta_{S i}}{\partial \theta_{0 j}}=\delta_{i j}-\partial_{i} \partial_{j} \Psi\left(f_{K}(\chi) \theta_{0}, \chi_{S}\right),
$$


where

$$
\begin{aligned}
\Psi\left(x_{\perp}, \chi_{S}\right) & =2 \int_{0}^{\chi_{h}} g(\chi) V\left(x_{\perp}, \chi\right) \mathrm{d} \chi \\
g(\chi) & =f_{K}(\chi) \int_{\chi}^{\chi_{h}} \frac{f_{K}\left(\chi^{\prime}-\chi\right)}{f_{K}\left(\chi^{\prime}\right)} \mathcal{P}\left(\chi^{\prime}\right) \mathrm{d} \chi^{\prime},
\end{aligned}
$$

with $\chi_{h}$ the horizon radius and $\mathcal{P}(\chi)$ an assumed galaxy distribution. This could be the redshift distribution $\mathcal{P}(z(\chi))$ of faint blue galaxies, which is well described by Bartelmann and Schneider, 2001

$$
\mathcal{P}(z(\chi))=\frac{3}{2 z_{0}}\left(\frac{z}{z_{0}}\right)^{2} \exp \left[-\left(\frac{z}{z_{0}}\right)^{3 / 2}\right],
$$

where $z_{0}$ depends on the magnitude cutoff and the colour selection of the galaxy sample.

Further defining the convergence

$$
\kappa\left(\theta_{0}\right)=\frac{1}{2}\left(\partial_{1} \partial_{1} \Psi+\partial_{2} \partial_{2} \Psi\right)
$$

and shear

$$
\gamma\left(\theta_{0}\right)=\gamma_{1}+i \gamma_{2}=\frac{1}{2}\left(\partial_{1} \partial_{1} \Psi-\partial_{2} \partial_{2} \Psi\right)+i \partial_{1} \partial_{2} \Psi
$$

the Jacobian matrix can be rewritten as

$$
A(\theta)=(1-\kappa)\left(\begin{array}{ll}
1 & 0 \\
0 & 1
\end{array}\right)-\left(\begin{array}{cc}
\gamma_{1} & \gamma_{2} \\
\gamma_{2} & -\gamma_{1}
\end{array}\right)
$$

The convergence thus isotropically magnifies the angular size in the neighborhood of $\theta$, while the shear causes anisotropy in the mapping. Using the Poisson Equation 2.25, the convergence can be expressed as a function of overdensity $\delta$ as

$$
\kappa\left(\theta_{0}\right)=\frac{3}{2} H_{0}^{2} \Omega_{\mathrm{FDM}} \int_{0}^{\chi_{h}} \frac{g(\chi)}{a(\chi)} \delta\left(f_{K}(\chi) \theta_{0}, \chi\right) \mathrm{d} \chi .
$$

Finally, expanding the convergence in multipoles $\kappa_{l m}$, the convergence power spectrum $P_{l}^{k}$ defined via

$$
\left\langle\kappa_{l m} \kappa_{l^{\prime} m^{\prime}}\right\rangle=\delta_{l l^{\prime}} \delta_{m m^{\prime}} P_{l}^{k}
$$

can be approximated by

$$
P_{l}^{k}=\frac{9}{4} \Omega_{\mathrm{FDM}}^{2} H_{0}^{4} \int_{0}^{\chi_{h}}\left(\frac{g(\chi)}{a(\chi) f_{k}(\chi)}\right)^{2} P\left(l / f_{K}(\chi), z\right),
$$


where $P\left(l / f_{K}(\chi), z\right)$ is the matter power spectrum. This formula was used in Marsh et al. 2012 in order to estimate the suppression of $P_{l}^{k}$ as a direct consequence of the suppression in $P\left(l / f_{K}(\chi), z\right)$ for large $l$ if the growth rate is partially reduced due to an FDM component in the dark sector [Marsh and Ferreira, 2010]. Marsh et al. 2012 additionally investigate possible degeneracies with relativistic neutrinos and dark energy models. As only scales above the Jeans length are observed by their assumed large future survey, the suppression does not depend on the FDM mass, but only on the FDM fraction to CDM. They conclude that with current and next generation galaxy surveys alone, it should be possible to unambiguously detect FDM if contributing at least a few percent to the overall DM density.

\subsubsection{Black Hole Superradiance}

Superradiance describes the exponential wave amplification of bosonic fields around rotating black holes $(\mathrm{BH})$. The interaction of the FDM wavefunction with the central BH results in massive scalar FDM clouds distributed similarly to the electron probability density within the hydrogen atom. This gravitational macroscopic atom configuration resonantly extracts angular momentum from the spinning $\mathrm{BH}$ if the FDM Compton wavelength is comparable to the Schwarzschild radius of the $\mathrm{BH}$ with mass $M_{\mathrm{BH}}$. This leads to characteristic exclusion regions in the BH mass-spin Regge plane Arvanitaki and Dubovsky, 2011; Brito et al., 2015; Endlich and Penco, 2017; Stott and Marsh, 2018].

In this section we use natural units setting $c=\hbar=G=1$. In order to avoid confusion with the magnetic quantum number $m$, we denote the FDM mass by $\mu$. Following the standard textbook by Wald [1984], we start explaining superradiance by stating that the spacetime around a spinning $\mathrm{BH}$ is characterized by the Kerr metric

$$
\begin{aligned}
\mathrm{d} s_{\text {Kerr }}^{2}= & -\left(1-\frac{2 M_{\mathrm{BH}} r}{\Sigma}\right) \mathrm{d} t^{2}-\frac{4 M_{\mathrm{BH}} a r \sin ^{2} \theta}{\Sigma} \mathrm{d} t \mathrm{~d} \phi+\frac{\Sigma}{\Delta} \mathrm{d} r^{2} \\
& +\Sigma \mathrm{d} \theta^{2}+\frac{\left(r^{2}+a^{2}\right)^{2}-a^{2} \Delta \sin ^{2} \theta}{\Sigma} \sin ^{2} \theta \mathrm{d} \phi^{2}
\end{aligned}
$$

with

$$
\begin{aligned}
\Sigma & =r^{2}+a^{2} \cos ^{2} \theta, \\
\Delta & =\left(r-r_{+}\right)\left(r-r_{-}\right), \\
r_{ \pm} & =M_{\mathrm{BH}} \pm \sqrt{M_{\mathrm{BH}}^{2}-a^{2}}
\end{aligned}
$$

and $a=J / M_{\mathrm{BH}}$, where $J$ is the BH's spin. The larger root of $\Delta, r_{+}$, defines the physical event horizon with area $A_{K}=8 \pi M_{\mathrm{BH}} r_{+}$, while $r_{-}$is a Cauchy horizon. They coincide in the extremal limit $a \rightarrow M_{\mathrm{BH}}$. The ergoregion is the region bounded by the outer horizon and the static surface at which $g_{t t}=0$ with radius

$$
r_{\text {ergo }}=M_{\mathrm{BH}}+\sqrt{M_{\mathrm{BH}}^{2}-a^{2} \cos ^{2} \theta} .
$$


Since $r_{\text {ergo }}=r_{+}$for $a \rightarrow 0$, the existence of this region is a defining property of a spinning $\mathrm{BH}$. An observer with zero angular momentum at infinity is dragged by the rotating $\mathrm{BH}$ and forced to co-rotate until reaching an angular momentum

$$
\Omega_{+}=\frac{a}{2 M_{\mathrm{BH}} r_{+}}
$$

at the physical event horizon. Indeed, a static observer with tangential vector proportional to the Killing vector $\xi^{\mu}=(1,0,0,0)$ associated with time invariance of the Kerr metric is not allowed within the ergoregion as $\xi^{\mu}$ becomes space-like there. The observer cannot stay still and is forced to co-rotate in the ergoregion. The Kerr metric is additionally invariant under rotation in $\phi$-direction. The linear combination of associated Killing vectors

$$
\chi=\partial_{t}+\Omega_{+} \partial_{\phi}
$$

is normal to the horizon and null there. The event horizon at $r_{+}$can thus be interpreted as rotating with angular velocity $\Omega_{+}$. Since $\xi^{\mu}$ becomes space-like in the ergoregion, the energy

$$
E=-p^{\mu} \xi_{\mu}
$$

of a test particle with four momentum $p^{\mu}$ can be negative there. By absorbing it, the $\mathrm{BH}$ will therefore lose energy. This process was first described by Penrose and Floyd 1971]. From

$$
0>p^{\mu} \chi_{\mu}=-E+\Omega_{+} L \quad \rightarrow \quad L<E / \Omega_{+}
$$

with $L=p^{\mu} \xi_{\mu}$. A particle with negative energy thus carries negative angular momentum. Then, absorption of the test particle not only reduces $M_{\mathrm{BH}}$ by $\delta M=E$ but also its spin $J$ by $\delta J=L$. Once all rotational energy

$$
\Omega_{+} \delta J=M_{\mathrm{BH}}-M_{\mathrm{irr}}=\delta M_{\mathrm{BH}}
$$

was extracted from the $\mathrm{BH}$, its background metric reduces to the Schwarzschild solution without an ergoregion and the Penrose process stops. Equation 2.118 then implies a minimal irreducible BH mass Christodoulou, 1970

$$
M_{\mathrm{irr}}^{2}=\frac{1}{2}\left[M_{\mathrm{BH}}^{2}+\sqrt{M_{\mathrm{BH}}^{4}-J^{2}}\right]=\frac{1}{4}\left(r_{+}^{2}+a^{2}\right)=\frac{1}{16 \pi} A_{S}
$$

proportional to the Schwarzschild BH area $A_{S}$ that ensures the validity of the area theorem.

While the Penrose process verifies the possibility of extracting the maximum amount of energy from a rotating $\mathrm{BH}$ permitted by the area theorem, it itself is not a practical energy extraction method. Its wave analog, called superradiant scattering, is physically more relevant. If an incident scalar wave

$$
\Psi=\exp [-i \omega t+i m \phi] f(r, \theta)+\text { h.c. }
$$


with frequency $\omega$ and azimuthal number $m$, which obeys the Klein-Gordon Equation 2.4. reaches the $\mathrm{BH}$, part of the wave will be transmitted and thus absorbed by the $\mathrm{BH}$. The rest will be reflected. Typically, the absorbed wave will carry positive energy reducing the energy transported by the reflected wave with respect to the incident wave. As for particles within the Penrose process, this is not necessarily true for rotating BHs. Contracting the stress tensor $T_{\mu \nu}$ corresponding to the scalar field $\Psi$ with $\xi_{\mu}$ yields a conserved energy current

$$
J_{\mu}=-T_{\mu \nu} \xi^{\nu}
$$

By Gauss's law the difference between the incoming and outgoing energies is equal to the integrated flux

$$
F=-\left\langle J_{\mu} \chi^{\mu}\right\rangle=\frac{1}{2} \omega\left(\omega-m \Omega_{+}\right)|f(r, \theta)|^{2}
$$

of $J_{\mu}$ on the horizon, where the brackets denote time averaging. If

$$
0<\omega<m \Omega_{+}
$$

this flux is negative and superradiance occurs, amplifying the energy of the outgoing wave. Alternatively, Equation 2.124 can also be derived from the area theorem [Bekenstein, 1973].

As a side remark, superradiant scattering is the classical limit of the stimulated emission associated with the spontaneous particle creation near a Kerr BH [Starobinsky, 1973, Unruh, 1974; Wald, 1976]. This means that initial bosonic quantum fluctuations around the Kerr BH can be macroscopically amplified by superradiance without the need for an initially non-vanishing energy density. This is comparable to the emission of a thermal spectrum of particles, called Hawking radiation, near a Schwarzschild BH. Studying FDM, we anyways assume a non-vanishing energy density of scalar fields around Kerr BHs.

The Klein-Gordon equation in a Kerr BH background permits separation of variables of a massive scalar field Brill et al., 1972]:

$$
\psi=\sum_{n, l, m} \exp \left[-i \omega_{n l m} t+i m \phi\right] S_{n l m}(\theta) R_{n l m}(r)+\text { h.c. }
$$

obeying

$$
\frac{1}{\sin (\theta)} \partial_{\theta}\left(\sin (\theta) \partial_{\theta} S_{n l m}\right)+\left[a^{2}\left(\omega_{n l m}^{2}-\mu^{2}\right) \cos ^{2} \theta-\frac{m^{2}}{\sin ^{2} \theta}+\Lambda_{l m}\right] S_{n l m}=0,
$$

and

$$
\begin{aligned}
& \left(\omega_{n l m}^{2}\left(r^{2}+a^{2}\right)^{2}-4 a M_{\mathrm{BH}} r m \omega_{n l m}+a^{2} m^{2}-\Delta\left(a^{2} \omega_{n l m}^{2}+\mu^{2} r^{2}+\Lambda_{l m}\right)\right) R_{n l m} \\
& \quad+\Delta \partial_{r}\left(\Delta \partial_{r} R_{n l m}\right)=0 .
\end{aligned}
$$


The angular Equation 2.126 is the oblate spheroidal angular wave equation Abramowitz, 1974. Expanding the angular eigenvalues

$$
\Lambda_{l m}=l(l+1)+\sum_{k=1}^{\infty} f_{k} c^{k}
$$

as a power series in $c=a^{2}\left(\omega^{2}-\mu^{2}\right)$, its solutions were constructed by Zouros and Eardley 1979 and Dolan 2007. Defining

$$
\tilde{R}=\sqrt{r^{2}+a^{2}} R \quad \text { and } \quad \mathrm{d} r_{\star}=\left(r^{2}+a^{2}\right) \Delta^{-1} \mathrm{~d} r,
$$

the radial Equation 2.127 can be written as a Schrödinger equation

$$
\partial_{r_{\star}}^{2} \tilde{R}+\left[\omega^{2}-V(\omega)\right] \tilde{R}=0
$$

with an effective potential [Zouros and Eardley, 1979]

$$
\begin{aligned}
V(\omega)= & \frac{\Delta \mu^{2}}{r^{2}+a^{2}}+\frac{4 M_{\mathrm{BH}} r a m \omega-a^{2} m^{2}+\Delta\left[\Lambda_{l m}+\left(\omega^{2}-\mu^{2}\right) a^{2}\right]}{\left(r^{2}+a^{2}\right)^{2}} \\
& +\frac{\Delta\left(3 r^{2}-4 M_{\mathrm{BH}} r+a^{2}\right)}{\left(r^{2}+a^{2}\right)^{3}}-\frac{3 \Delta^{2} r^{2}}{\left(r^{2}+a^{2}\right)^{4}}
\end{aligned}
$$

The asymptotic solutions of an incoming wave at the BH horizon and an outgoing wave at spatial infinity are Teukolsky, 1972

$$
\begin{aligned}
& R(r) \sim \exp \left[-i\left(\omega-m \Omega_{+}\right) r_{\star}\right] \quad \text { for } r \rightarrow r_{+}\left(r_{\star} \rightarrow-\infty\right), \\
& R(r) \sim \exp \left[-k r_{\star}\right] / r \text { for } r \rightarrow \infty\left(r_{\star} \rightarrow+\infty\right),
\end{aligned}
$$

with the FDM momentum $k=\sqrt{\mu^{2}-\omega^{2}}$, showing that the wave is confined around the $\mathrm{BH}$ for

$$
0<\omega<\mu \text {. }
$$

The solution for the radial equation has been computed both in the small coupling limit $\alpha=M_{B H} \mu \ll 1$ [Detweiler, 1980] and for $\alpha \gg 1$ [Zouros and Eardley, 1979]. Since $\alpha \ll 1$ implies a Schwarzschild radius much smaller then the Compton wavelength of the scalar wave, the computations in this regime are comparable to those of the hydrogen atom. For large $r$ far away from the $\mathrm{BH}$ the radial component is therefore

$$
R_{\mathrm{far}}(r)=(2 k r)^{l} \exp [-k r] U\left(l+1-\frac{\alpha^{2}}{M_{\mathrm{BH}} k}, 2 l+2,2 k r\right),
$$

where $U$ is the confluent hypergeometric function of the second kind [Abramowitz, 1974]. For the hydrogen atom $\bar{n}=\alpha^{2} / M_{\mathrm{BH}} k=l+1+n$ would be an integer depending on the principal $n$ and the azimuthal quantum number $l$ ensuring regularity of the electron 
wavefunction at the origin. Here, regularity has to be enforced at the finite BH horizon. In the assumed limit $\alpha \ll 1$ this introduces a small complex correction to the above relation and

$$
\omega \simeq \mu \sqrt{1-\alpha^{2} / \bar{n}^{2}} \simeq \mu\left(1-\alpha^{2} / 2 \bar{n}^{2}\right)
$$

is only approximately true. The radial component near the horizon takes the form

$$
R_{\text {near }}(r)=\left(\frac{r-r_{+}}{r-r_{-}}\right)^{-i P}{ }_{2} F_{1}\left(-l, l+1,1+2 i P, \frac{r-r_{-}}{r_{+}-r_{-}}\right)
$$

where

$$
P=2 r_{+} \frac{\omega-m \Omega_{+}}{r_{+}-r_{-}}
$$

and ${ }_{2} F_{1}$ is Gauss's hypergeometric function. For small enough $\alpha \ll 1$ both Equation 2.134 and Equation 2.136 are valid at an intermediate radius $r_{0}$ and can be matched there |Detweiler, 1980|. While the real part of the matching frequency is still well approximated by Equation 2.135, the imaginary correction is approximately given by

$$
\begin{aligned}
\omega_{I}= & 2 \mu \alpha^{4 l+4} r_{+}\left(m \Omega_{+}-\mu\right) \frac{2^{4 l+2}(2 l+n+1)}{(l+n+1)^{2 l+4} n !}\left(\frac{l !}{(2 l) !(2 l+1) !}\right)^{2} \\
& \times \prod_{j=1}^{l}\left(j^{2}\left(1-\frac{a^{2}}{M_{\mathrm{BH}}^{2}}\right)+4 r_{+}^{2}\left(m \Omega_{+}-\mu\right)^{2}\right) .
\end{aligned}
$$

It is positive for $\omega \simeq \mu<m \Omega_{+}$signaling an unstable mode in the superradiant regime. Since $\omega_{I}$ decreases exponentially with $l$, it is largest for the lowest possible $l=m$ fulfilling the superradiant condition in Equation 2.124. This mode is therefore the fastest growing mode with growth time $\tau=\omega_{I}^{-1}$. There is also a mild dependency on $n$. A slightly different, semi-analytical, matching was employed by Arvanitaki and Dubovsky [2011]. Their instability rates correspond better to numerically obtained results [Dolan, 2007], but yield the same qualitative behaviour. In particular the maximum rate,

$$
\omega_{I, \max } \simeq 1.5 \cdot 10^{-7} M_{\mathrm{BH}}^{-1},
$$

is found for $\alpha \simeq 0.42$ and an almost maximally spinning $\mathrm{BH} a=0.99 M_{\mathrm{BH}}$.

The strong coupling regime $\alpha \gg 1$ was investigated by Zouros and Eardley [1979] using a WKB approximation. The superradiance rate is then determined by the tunneling probability through the potential barrier at $r_{\star}=0$ of the effective potential in Equation 2.131 that separates the bound Keplerian orbits from the near horizon region where superradiant amplification takes place. Figure 2 in Zouros and Eardley [1979] shows the typical form of the effective potential $V\left(r_{\star}\right)$. The flux through the BH horizon as given in Equation 2.123 is therefore exponentially suppressed for $\alpha \gg 1$ and

$$
\omega_{I} \sim\left(m \Omega_{+}-\omega\right) \exp [-2 I]
$$


depends on the tunneling integral

$$
I=\int_{\Delta r_{\star}(r)} \sqrt{V\left(r_{\star}\right)-\omega^{2}} \mathrm{~d} r_{\star}=\int_{\Delta r} \sqrt{V(r)-\omega^{2}}\left(r^{2}+a^{2}\right) \Delta^{-1} \mathrm{~d} r
$$

over the classically forbidden region $\Delta r$. Equation 2.141 can be integrated numerically. It was analytically calculated by Zouros and Eardley [1979] for $\mu=\omega=m \Omega_{+}$right at the boundary of superradient scattering. Under these assumptions $I$ defines the upper envelope of the family of superradiant rates for different levels $l$ Arvanitaki and Dubovsky, 2011. The integral is found to be a decreasing function of $a$ and $P=\omega / m \Omega_{+}$. For $P=1$, the limiting values are Zouros and Eardley, 1979

$$
\begin{aligned}
& I \rightarrow \alpha\left(8 M_{\mathrm{BH}} / a\right)\left[\ln \left(8 M_{\mathrm{BH}} / a\right)-1\right] \quad \text { as } \quad a / M_{\mathrm{BH}} \rightarrow 0, \\
& I \rightarrow \alpha(2-\sqrt{2}) \pi \simeq 1.84 \alpha \quad \text { as } \quad a / M_{\mathrm{BH}} \rightarrow 1 .
\end{aligned}
$$

Matching the prefactor with Equation 2.139 sets the approximate maximum superradiant scattering rate in the large coupling limit $\alpha \gg 1$ to Arvanitaki and Dubovsky, 2011

$$
\omega_{I, \max } \simeq 10^{-7} M_{\mathrm{BH}}^{-1} \exp [-2 \pi \alpha(2-\sqrt{2})] \simeq 10^{-7} M_{\mathrm{BH}}^{-1} \exp [-3.7 \alpha],
$$

in agreement with results obtained by Zouros and Eardley [1979] and Gaina [1989].

Due to the exponential suppression for large $\alpha \gg 1$ and the polynomial suppression for small $\alpha \ll 1$, in order for superradiance to be physically relevant we therefore need $\alpha \sim 1$. Then,

$$
\frac{M_{\mathrm{BH}}}{\mu} \sim\left(\frac{M_{\mathrm{BH}}}{m_{p}}\right)^{2} \sim 10^{96}\left(\frac{M_{\mathrm{BH}}}{10^{10} M_{\odot}}\right)^{2}
$$

where $m_{p}$ denotes the Planck mass. Thus, $\sim 2 \times 10^{2}$ e-foldings of superradiance are needed to form an FDM cloud comparable to BH mass. That corresponds to a time scale

$$
t_{\mathrm{SR}} \sim 2 \times 10^{2} \omega_{I, \text { max }}^{-1} \sim 10^{14}\left(\frac{M_{\mathrm{BH}}}{10^{10} M_{\odot}}\right) s \sim 10^{7}\left(\frac{M_{\mathrm{BH}}}{10^{10} M_{\odot}}\right) \mathrm{yr},
$$

much shorter than the Hubble time scale $t_{H} \sim 10^{10} \mathrm{yr}$ or the Eddington time $t_{E} \sim 4 \times 10^{8} \mathrm{yr}$ for $\mathrm{BH}$ smooth mass accretion without significant mergers but seven orders of magnitude larger than the dynamical time scale of the $\mathrm{BH} t_{\mathrm{BH}} \sim\left(M_{\mathrm{BH}} / 10^{10} M_{\odot}\right)$ yr Arvanitaki and Dubovsky, 2011. Figure 2 in Arvanitaki and Dubovsky 2011 depicts the band around $\alpha \sim 1$ as a function of FDM and BH mass for which $t_{\mathrm{SR}}$ is smaller than $t_{H}$ or $t_{E}$. This can serve as a zeroth order estimate for parameter ranges with observable superradiant phenomenology, the main limitation being the assumption that all $\mathrm{BH}$ are maximally spinning.

The analysis can be refined by calculating FDM mass dependent exclusion regions in the BH spin verses mass plane, called the Regge plot. Observed BHs with parameters 
inside the forbidden region then exclude the corresponding FDM mass. Representative Regge plots over a large range of FDM masses are shown in Stott and Marsh [2018].

The exclusion region can be understood by considering an initially rapidly spinning $\mathrm{BH}$ with $a \lesssim M_{\mathrm{BH}}$. From the above discussion, assuming $\alpha \lesssim 1 / 2$, the most unstable superradiant level is given by $l=m=1$. Due to superradiance this level is exponentially amplified with a rate given in Equation 2.138. This rapidly decreases the $\mathrm{BH}$ spin until the superradiance condition Equation 2.124 is not satisfied anymore. At this point the $l=m=2$ level becomes most unstable. However, due to its smaller rate, spin is less effectively extracted from the $\mathrm{BH}$. Additionally, for low spins the $l=m=1$ level is now stable and starts spinning up the $\mathrm{BH}$. There is thus an intermediate time interval during which the $\mathrm{BH}$ spin stays roughly constant while mass is transferred from the $l=m=1$ to the $l=m=2$ level until the occupation number from the former drops below the occupation number of the latter decreased by the ratio of there respective superradiance rates. At that point the $\mathrm{BH}$ spin starts decreasing until the second level saturates as well. In this way, successively highers level become important until their superradiance time scales become comparable to $t_{H}$ or $t_{E}$. BHs with smaller spin are then not able to exclude the considered FDM mass. Non-linear effects and smooth accretion tend to slightly enhance the duration during which the $\mathrm{BH}$ spin stays approximately constant Arvanitaki and Dubovsky, 2011]. These stability points constitute the so called Regge trajectories in the Regge plane that should be traced by observed BHs.

Using data from observed spinning super massive BHs, Stott and Marsh [2018] could exclude FDM masses

$$
7 \times 10^{-20} \mathrm{eV}<\mu<1 \times 10^{-16} \mathrm{eV}
$$

at a $95 \%$ confidence level in good agreement with earlier results [Cardoso et al., 2018]. Superradiance thus constraints a complimentary mass range with respect to the previous observational methods and leaves only an order of magnitude gap around $m_{22} \sim 10^{2}$ that can potentially be closed within the next years.

The presence of an FDM cloud around a BH can also be observed by its associated gravitational wave signal due to FDM particle transitions between different levels and FDM particle annihilation into gravitons Arvanitaki and Dubovsky, 2011. Prospects for LISA suggest an exclusion region comparable to Equation 2.146 [Brito et al., 2017a. b]. The expected gravitational wave signatures of FDM clouds around BHs in binary inspirals has been investigated by Baumann et al. 2018.

\subsection{Small-Scale Tensions}

Standard $\Lambda$ CDM cosmology has proven extremely successful over the last decades. However, CDM has vanishing equation of state and sound speed $\left(w=c_{s}=0\right)$ and thus clusters on all scales. This leads to a number of identified small-scale problems Bullock and Boylan-Kolchin, 2017; Buckley and Peter, 2017. 
The missing satellite problem arises from the over-prediction of the abundance of satellite halos of a particular velocity dispersion in numerical simulation compared to the number of galaxies of similar velocities that have actually been observed in the Milky Way Klypin et al., 1999; Moore et al., 1999; Springel et al., 2008, Bullock, 2010. High-resolution cosmological simulations of Milky Way-sized halos show a continuously increasing subhalo mass function towards lighter subhalos with no break down to the numerical convergence as a consequence of the self-similar gravitational collapse of CDM. Thus thousands of subhalos heavy enough to support molecular cooling and thus star formation are expected while less then a hundred satellite galaxies are observed around the Milky Way. The observed stellar mass functions of field galaxies and satellite galaxies in the Local Group are therefore much flatter at low masses than predicted CDM HMFs. The discrepancy could be alleviated if star formation within CDM halos becomes increasingly inefficient for low halo masses and stops at a minimum mass due to suppressed gas accretion in halos with strong reionization UV feedback $\left(M_{\text {vir }} \lesssim 10^{9} M_{\odot}\right)$ [Efstathiou, 1992; Bullock et al., 2000; Sawala et al. 2016 or insufficient atomic cooling in the early universe $\left(M_{\text {vir }} \lesssim 10^{8} M_{\odot}\right)$ [Rees and Ostriker, 1977]. If true, a simple abundance matching as discussed above could successfully relate simulated CDM halo masses $M_{\text {vir }}$ with observed star masses $M_{\star}\left(M_{\text {vir }}\right)$ Garrison-Kimmel et al., 2014.

The cusp core problem [Flores and Primack, 1994; Moore, 1994] refers to the fact that DM only simulations show cuspy and dense NFW-like profiles with an inner slope of $\gamma \simeq 0.8-1.4$ Navarro et al. 2009], while measured rotation curves of low mass CDM dominated galaxies prefer fits with constant CDM density cores $\gamma \simeq 0.0-0.5$ McGaugh et al., 2001; Simon et al., 2005; Walker and Peñarrubia, 2011; Alam et al., 2002; Oman et al., 2015] as parametrized by Burkert [1995].

If abundance matching indeed solves the missing satellite problem, then the inferred central masses of Milky Way satellites should coincide with the central masses of the most massive subhalos of simulated Milky Way-sized halos. Comparing data with simulations [Springel et al., 2008; Diemand et al. 2008], Boylan-Kolchin et al. 2011] concluded that the centers of the most massive CDM subhalos were systematically too dense in order to host the detected bright Milky Way satellites. Thus, the so-called too-big-to-fail problem describes the apparent insufficient star formation within the most massive satellites that is not seen in lower mass halos. Generally, the inferred central masses of galaxies with $10^{5} \lesssim M_{\star} / M_{\odot} \lesssim 10^{8}$ are $\sim 50 \%$ smaller than expected from CDM simulations Bullock and Boylan-Kolchin, 2017.

A variety of possible solutions have been proposed to overcome these shortcomings. Baryonic feedback from supernova explosion might solve or at least reduce the discrepancies by transporting CDM from within the central halo region outwards Mashchenko et al., 2008; Governato et al., 2012; Pontzen and Governato, 2014; Madau et al., 2014; Oñorbe et al., 2015; Read and Erkal, 2018]. On the one hand, this is only sufficient to form cores in halos with enough star formation. On the other hand, too high stellar mass fractions result in excess central masses that can compensate supernova feedback and drag CDM back in. Large hydrodynamical simulations suggest that feedback can potentially solve the cusp-core and too-big-to-fail problem for mass ratios $M_{\star} / M_{\text {vir }} \sim 0.005$ [Tollet et al., 2016; 
Fitts et al., 2017; Wetzel et al., 2016; Hopkins et al., 2018; Garrison-Kimmel et al., 2017, found in bright dwarfs with $M_{\text {star }} \gtrsim 10^{6} M_{\odot}$ and $M_{\text {vir }} \gtrsim 10^{10} M_{\odot}$, but is insufficient for classical dwarfs and ultra-faint dwarfs with orders of magnitude lower mass ratios. Even though results from various simulations start to converge, they strongly depend on the adopted parameters modeling galaxy formation. Additionally, environmental interactions between the satellites and their host can alleviate small-scale tensions on all satellite mass scales Zolotov et al., 2012; Arraki et al., 2013; Dutton et al., 2016; Wetzel et al., 2016, Sawala et al., 2016]. Examples are tidal and ram pressure stripping or disk shocking.

Another approach is to modify the CDM paradigm. Warm dark matter (WDM) could erase density perturbations below its effective free-streaming length Bode et al., 2001; Viel et al., 2005, Schneider et al., 2012,

$$
\lambda_{\mathrm{fs}}^{\mathrm{eff}} \simeq 49\left(\frac{m_{\mathrm{WDM}}}{\mathrm{keV}}\right)^{-1.11}\left(\frac{\Omega_{\mathrm{DM}}}{0.25}\right)^{0.11}\left(\frac{h}{0.7}\right)^{1.22} \mathrm{kpc} / \mathrm{h} .
$$

On the one hand, this leads to a very distinctive cutoff in the matter power spectrum conventionally characterized by the wave number at which the ratio between the linear WDM and CDM power drops below one half. The corresponding wavelength $\lambda_{1 / 2} \simeq 14 \lambda_{\mathrm{fs}}$ is the scale below which modified growth of linear perturbations strongly suppresses halo formation [Schneider et al. 2012]. This can potentially solve the missing satellite and too-big-to-fail problem for WDM masses $\simeq 3 \mathrm{keV}$ [Lovell et al., 2014] still allowed by Ly $\alpha$ measurements Baur et al. 2016. On the other hand, $\mathcal{O}(\mathrm{keV})$ WDM masses produce dwarf galaxy cores with radii $r_{c} \sim 10 \mathrm{pc}$, two orders of magnitude smaller in size than observed for large Milky Way satellites [Walker and Peñarrubia, 2011]. This gives rise to the catch -22 problem [Macciò et al., 2012a b; Schneider et al., 2014].

FDM masses favored by observations suggesting cored halos will be discussed in the next section. Comparing them to the above outlined lower bounds, FDM starts to have a catch -22 problem as well. Due to the very different suppression mechanisms of WDM and FDM density perturbations, it is by far not as severe as in the WDM scenario.

Strong self-interactions within the DM sector could also change small-scale clustering Kaplinghat et al., 2016 but would require self-interactions orders of magnitude above those expected for weakly interacting massive particles [Spergel and Steinhardt, 2000]. Numerical simulations suggest that scattering cross sections per unit mass $\sigma / m \sim 0.5-10 \mathrm{~cm}^{2} / \mathrm{g}$ can produce large enough cores to alleviate the cusp-core and too-big-to-fail problems discussed above |Vogelsberger et al., 2012, Elbert et al., 2015, Fry et al., 2015. These high cross sections are marginally excluded by the observed central DM densities in galaxy clusters Elbert et al. 2018; Kaplinghat et al., 2016]. Velocity dependent cross sections can reconcile this tension.

\subsection{Halo Density Profiles}

Using stellar spectroscopic data from dwarf spheroidal (dSph) Milky Way satellites, Walker and Peñarrubia 2011] infer on the radial slopes of their mass profiles. They conclude that 
both investigated dSph galaxies Fornax and Sculptor have slopes compatible with inner cores of constant density within the central few-hundred parsecs of each galaxy, ruling out NFW-like cusps with high statistical significance and providing a lower limit on the core sizes. However, these results are still under debate, since other analyses did not yield significant exclusions for cuspy profiles Breddels and Helmi, 2013; Richardson and Fairbairn, 2014 Strigari et al., 2014. If true, the non-observation would again provide a lower limit on the FDM mass. Here, we summaries results obtained by taking the results of Walker and Peñarrubia [2011] at face value. An upper limit on the core size can be inferred from the requirement that dynamical friction time scales for both dSphs Gerhard and Spergel, 1992

$$
t_{\text {fric }} \simeq \frac{10^{10} \mathrm{yr}}{\ln \Lambda}\left(\frac{r}{60 \mathrm{kpc}}\right)^{2}\left(\frac{v_{c}}{220 \mathrm{~km} / \mathrm{s}}\right)\left(\frac{2 \times 10^{10} M_{\odot}}{M}\right)
$$

should not be too small compared to the Hubble time, restricting the dSph masses to $M \lesssim 10^{10} M_{\odot}$.

Fitting an analytic radial density profile

$$
\rho(r)=\Theta\left(r_{\epsilon}-r\right) \rho_{\mathrm{sol}}(r)+\Theta\left(r-r_{\epsilon}\right) \rho_{\mathrm{NFW}}(r)
$$

to the data, Marsh and Pop 2015] conclude that it can be explained purely by FDM physics if $m_{22} \lesssim 1.1$ at $95 \%$ confidence level. Here, $\rho_{\text {sol }}(r)$ is the soliton density profile found in numerical simulations [Schive et al., 2014a] discussed in the next chapter, and $r_{\epsilon}$ defines the radius at which the inner solitonic profile continuously turns into an NFW outer tail. Numerically, it is found that $r_{\epsilon} \simeq 3.5 r_{c}$, where $r_{c}$ denotes the solitonic core radius Mocz et al., 2017]. Performing a Jeans analysis for the dominant intermediate metallicity stellar population of Fornax, Schive et al. 2014a find $m_{22} \simeq 0.81_{-1.7}^{+1.6}(1 \sigma)$ implying a solitonic core radius $r_{c} \simeq 0.92_{-0.11}^{+0.10} \mathrm{kpc}$. This FDM mass produces a Milky Way core with mass $M_{c} \simeq 2 \times 10^{9} M_{\odot}$, radius $r_{c} \simeq 180 \mathrm{pc}$ and potential depth corresponding to a line-of-sight velocity dispersion $\sigma_{\|} \simeq 115 \mathrm{~km} / \mathrm{s}$ consistent with data suggesting a Milky Way bulge with $r_{c} \simeq 200 \mathrm{pc}$ and $\sigma_{\|} \simeq 110 \mathrm{~km} / \mathrm{s}$ [Schive et al. 2014a; Minniti, 1996; Rich et al., 2007; Ness et al., 2013. The analysis of the FDM implications on the Milky Way bulge properties was refined by Martino et al. [2018], confirming the previously found best fit FDM mass. Extending the Jeans analysis to a larger data set of eight classical dSphs, Chen et al. [2017] obtain $m_{22} \simeq 1.18_{-0.24}^{+0.28}$ and $m_{22} \simeq 1.79_{-0.33}^{+0.35}$ for the two data sets studied [Walker et al., 2007, 2009]. Fitting the luminosity-averaged velocity dispersion of the individual chemodynamical components of Fornax and Sculptor, González-Morales et al. 2017] find a more restrictive upper bound $m_{22} \lesssim 0.4$ at $97.5 \%$ confidence level. The rotation curves of high-resolution low surface brightness and SPARC galaxies can be reproduced if FDM is even lighter with $m_{22} \simeq 0.0554$ [Bernal et al., 2017]. Investigating the longevity of the cold clump in Ursa Minor and the rapid orbital decay of the globular clusters in Fornax and dwarf ellipticals, Lora et al. 2012] obtain a best fit FDM mass $0.3<m_{22}<1.0$. Measurements of the half-light mass in the ultra-faint dwarf galaxies Draco II and Triangulum II imply $3.7<m_{22}<5.6$ [Calabrese and Spergel, 2016]. The Msigma relation between the mass of super massive black holes in galaxies and the velocity 
dispersions of their bulge can be explained if $m_{22} \simeq 5$ Lee et al., 2015]. Employing a quasi particle approach to simulate the granular structure of FDM halos [Hui et al., 2017], Amorisco and Loeb [2018] numerically investigate the thickening of thin stellar streams. Comparing their results to Milky Way data, they obtain a lower limit $m_{22}>1.5$. We will comment on potential limitations of their effective FDM approach below. An additional component of heavier FDM with mass $m_{22} \simeq(3.2 \pm 0.62) \times 10^{4}$ can also account for the observed compact dark masses at the centers of globular clusters around the Milky Way Emami et al. 2018. Finally, it was claimed that FDM with $m_{22} \sim 1$ can produce caustic rings observable in the Milky Way Chakrabarty and Sikivie, 2018, Banik et al., 2017, Sikivie, 1998]. All these analyses on the Milky Way substructure coherently indicate a best fit FDM mass $m_{22} \sim 1$, while the above listed lower bounds, derived from large-scale structure formation, suggest $m_{22}>\mathcal{O}(10)$. Thus, FDM starts to have a similar catch -22 problem as WDM. This tension is strengthened when estimating survival times of Milky Way satellites. Using empirical data from Wolf et al. [2010], Du et al. [2018] conclude that the lightest satellites close to the Galactic center will only survive for more than one orbital time if the particle is as heavy as $m \simeq 2 \times 10^{-21} \mathrm{eV}$. We summarize their analysis and results in the next chapter.

Unfortunately, there is a caveat in the above analyses. It is empirically found that the product of the core density $\rho_{c}$ and the core radius $r_{c}$ is constant Kormendy and Freeman, 2004]. While Spano et al. [2007] and Donato et al. [2009 find $\rho_{c} r_{c}=140_{-30}^{+80} M_{\odot} \mathrm{pc}^{-2}$, Burkert 2015 obtain $\rho_{c} r_{c}=75_{-45}^{+85} M_{\odot} \mathrm{pc}^{-2}$. In Lin and Loeb 2016 interacting DM is considered. Assuming generic two-body interactions, it is shown that

$$
\rho_{c} r_{c}=75 M_{\odot} \mathrm{pc}^{-2} \times\left(\frac{M_{200}}{10^{10} M_{\odot}}\right)^{0.18}
$$

where the fiducial value $M_{200} \sim 10^{10} M_{\odot}$ is the mass within the radius $r_{200}$ interior to which the average density is 200 times the critical density of the universe Oñorbe et al., 2015. Equation 2.150 is obtained without any assumptions on the DM mass or interaction strength. The mild dependence on the halo mass naturally explains the deviation by a factor of two between the first two results above Equation 2.150 [Lin and Loeb, 2016].

For a power spectrum of initial density fluctuations that is a power law in wave number $k,\left|\delta_{k}\right|^{2} \sim k^{n}$, the density of bound object is related to its size by $\rho \sim r^{3(3+n) /(5+n)}$ Kormendy and Freeman, 2004. The above relation thus implies $n=-2$ in good agreement with $\Lambda$ CDM theory predicting $n=-2.1$ for halo masses of $10^{12} M_{\odot}$ [Shapiro and Iliev, 2002 .

On the contrary, FDM solitons admit a scaling $\rho_{c} \sim r_{c}^{-4}$. This let Deng et al. [2018] to conclude that the solution to the core-cusp problem is more likely due to either baryonic effects or some other type of DM interactions. Even if FDM does not solve the core-cusp problem, it might be responsible for some of the above summarized phenomenology. 


\section{Chapter 3}

\section{Fuzzy Dark Matter Simulations}

From the previous discussion it is obvious that full FDM simulations that take coherence effects into account also during the non-linear evolution of density perturbations can strengthen the lower bounds on the FDM mass and are imperative for studies of halo substructure. Fortunately, there is a growing numerical toolbox available to which the author contributed as described within the subsequent chapters. Generally, four main approaches have emerged, namely Eulerian finite difference [Widrow and Kaiser, 1993; Schive et al., 2014a; Schwabe et al., 2016; Kopp et al., 2017) and spectral [Woo and Chiueh, 2009; Mocz et al., 2018; Du et al., 2018; Edwards et al., 2018| methods, Lagrangian particle based schemes [Veltmaat and Niemeyer, 2016; Zhang et al., 2018b; Chan et al., 2018; Nori and Baldi, 2018] and semi-analytic methods [Du et al., 2016; Lin et al., 2018]. The Gaussian beam method described below extends the pool of Lagrangian discretizations for FDM simulations.

\subsection{Eulerian Grid Based Simulations}

Following Press et al. [2007], the Eulerian approach to solve the SP system is to provide initial information of the wavefunction at a finite set of fixed grid points. Spacial gradients

$$
\nabla \psi(x)=\lim _{\Delta x \rightarrow 0} \frac{\psi(x+\Delta x)-\psi(x)}{\Delta x}
$$

can then be evaluated between neighboring grid points by dropping the analytic limit. Since $\Delta x$ corresponds to the grid resolution - the distance between two points - the approximate calculation of gradients convergences to the analytic result as the grid spacing $\Delta x$ is decreased. Higher-order convergence can be achieved by using information from more neighboring points in a knowledgeable way. From the Madelung transformation defined in Equation 2.16, we can infer that $\psi$ changes significantly on de Broglie scales $\lambda=h / m v$, setting the spacial resolution requirement to $\Delta x \lesssim \lambda$.

Using the same discretization scheme in the temporal dimension, the wavefunction can 
be explicitly evolved in time

$$
\psi(t+\Delta t, x)=i\left[\frac{\hbar}{2 a^{2} m} \nabla^{2}-\frac{m}{\hbar} V\right] \Delta t+\psi(t, x) .
$$

Better convergence can be achieved by sub-dividing time steps in a favorable manner. One such scheme is the Runge-Kutta four algorithm employed for the soliton mergers below. In Equation 3.2, the Laplace operator $\nabla^{2}$ is discretized comparable to Equation 3.1. Due to the diffusive nature of the Schrödinger equation, it is unconditionally stable if the CourantFriedrichs-Lewy stability criterion

$$
\Delta t \leq \min \left[\frac{m}{6 \hbar} \Delta x^{2}, \frac{\hbar}{m|V|_{\max }}\right]
$$

is satisfied. Physically, this inequality requires time steps $\Delta t$ to be small compared to the coherence time scale $t_{c} \sim(\Delta x)^{2} m / \hbar \sim \hbar / m v^{2}$. Additionally, it has to be smaller than the time scale $t_{p} \sim \hbar / m V$ characterized by the potential $V$, such that information is transferred at most between adjacent grid points in each temporal iteration. The latter time scale is inversely proportional to the absolute value of the potential. If global time steps over a certain sub-region of the entire problem are preferred in numerical implementations, the strongest constraint, coming from the maximal absolute value $|V|_{\max }$, has to be fulfilled. In contrast, the SP system is unconditionally stable if implicit or pseudo spectral methods are used that redistribute information over the entire problem size in every time step. Then, the time step size is only limited by the desired minimum level of accuracy. However, implicit methods involve the construction of the inverse of a large square matrix $N \times N$, where $N$ is the number of grid points. This typically restricts this method to uniform rectangular grids with constant spacing and is difficult to parallelize for multi-processor applications.

Pseudo spectral methods take advantage of the fact that gradients become products of wave numbers in Fourier space. Thus, gradients can be calculated analytically without further numerical approximations. They are only present in the finite number of wave numbers $k_{i}$ matching the number of grid points in position space. While again restricted to uniform rectangular grids with constant spacing, discrete Fourier transformations can be efficiently calculated with numerical cost scaling as $N \log N$, and admit good scalability when parallelized on a large number of processors. On uniform grids, spectral methods are usually the preferred method. For example, within the Enzo code, the Poisson equation on the uniformly spaced root grid is calculated in this way. On higher non-uniform levels, more involved algorithms have to be employed.

The first FDM simulation numerically solving the SP system was presented by Widrow and Kaiser [1993]. The authors already pointed out that velocity dispersion in the classical phase-space distribution results in interference patterns on de Broglie scales clearly visible in the quantum mechanical probability distribution that is identified with the FDM density field. The classical CDM density is recovered when smoothed over multiple de Broglie wavelengths. Later, it was shown that this granular structure strongly fluctuates 
on dynamical time scales, while leaving almost no imprint in the gravitational potential [Hu et al., 2000]. Employing Cayley's implicit finite-difference scheme [Goldberg et al. 1967| for the Schrödinger equation and a fast Fourier transformation in order to solve for the gravitational potential, Widrow and Kaiser [1993] further prove that the SP system accurately reproduces non-linear dynamics during gravitational collapse by comparing the associated classical phase-space distribution with results from pure N-body simulations. Both reveal the same shell-crossing and multi-streaming characteristics after multiple freefall times. Additionally, the SP system does not produce caustics at shell-crossing as no point-like entities with definite trajectories are assumed and perturbation theory within the SP system does not produce negative densities Coles and Spencer, 2003; Szapudi and Kaiser, 2003. The SP system can thus also be seen as a regularization scheme for standard N-body systems. The correspondence between the SP and the Vlasov-Poisson system represented by CDM N-body techniques was further investigated in Uhlemann et al. [2014]; Uhlemann [2018]; Garny and Konstandin [2018]; Mocz et al. [2018]. It will be discussed in depth below. Widrow and Kaiser [1993] conclude their paper by presenting a two dimensional cosmological simulation. While density perturbations above the de Broglie wavelength evolve identically between FDM and CDM, the obtained density distributions clearly deviate on small scales.

Running three dimensional cosmological simulations by integrating the SP system with a pseudo spectral method, Woo and Chiueh [2009] showed that the growth of the initial CDM density perturbations is strongly suppressed below the Jeans scale $r_{J} \sim a^{1 / 4}$ while more extended overdensities increase with $a$ as expected from linear theory. This was confirmed by Veltmaat and Niemeyer 2016]. During non-linear gravitational collapse, halos are found to relax into virialized configurations by emitting pronounced density waves that transport excess angular momentum and kinetic energy into the IGM Woo and Chiueh, 2009]. This gravitational cooling, comparable to violent relaxation, has been thoroughly investigated by [Seidel and Suen, 1994; Guzmán and Ureña-López, 2004]. Similar dynamics were reported by [Schwabe et al., 2016]. The resulting radial profiles suggest NFW-like halo shapes similar to CDM scenarios. The granular structure on small scales, due to wave interference, could be recovered in the filaments and halos. Furthermore, identifying the gradient of the complex wavefunction with the systems velocity, Woo and Chiueh 2009] found a clear separation between linear infall in the outer regions and strong velocity dispersions in the inner regions of FDM halos where shell-crossing already happened. This strong shock front in the velocity field has no visible analog in the density field.

\subsection{Simulating Solitonic Cores in FDM Halos}

Employing a pseudo spectral method, the simulation was restricted to a uniform grid. The halos within the simulated one $\mathrm{Mpc} / h$ cosmological box could thus be resolved only down to comoving kpc. This limitation was overcome by using a finite difference scheme on an adaptively refined mesh Schive et al., 2014a]. With increased resolution a gravitationally self-bound core developed in the center of each halo. Apart from small perturbations, 
it was found to be the boson star or soliton previously studied as the ground state of a self-gravitating scalar field [Ruffini and Bonazzola, 1969; Guzmán and Ureña-López, 2004, Liebling and Palenzuela, 2017]. Its radial density profile is well approximated by [Schive et al., 2014b]

$$
\rho_{c}(r) \simeq \rho_{0}\left[1+0.091 \cdot\left(r / r_{c}\right)^{2}\right]^{-8}
$$

where $r_{c}$ is the radius at which the density drops to one-half its peak value and the central density is given by

$$
\rho_{0} \simeq 3.1 \times 10^{15}\left(\frac{2.5 \times 10^{-22} \mathrm{eV}}{m}\right)^{2}\left(\frac{\mathrm{kpc}}{r_{c}}\right)^{4} \frac{M_{\odot}}{\mathrm{Mpc}^{3}}
$$

The core mass $M_{c}$ is typically defined as the mass enclosed by $r_{c}$ and corresponds to roughly a fourth of the total soliton mass $M$ [Schive et al., 2014b]:

$$
\begin{aligned}
\frac{M_{c}}{M_{\odot}} & \simeq 0.237 \frac{M}{M_{\odot}} \\
& \simeq 8.64 \times 10^{6}\left(\frac{2.5 \times 10^{-22} \mathrm{eV}}{m}\right)^{2}\left(\frac{\mathrm{kpc}}{r_{c}}\right) \\
& \simeq 1.81 \times 10^{6}\left(\frac{2.5 \times 10^{-22} \mathrm{eV}}{m}\right)\left(\frac{E}{M}\right)^{1 / 2} \frac{\mathrm{s}}{\mathrm{km}}
\end{aligned}
$$

Schive et al. 2014b] show evidence for the same scaling relation between $M_{c}, E$, and $M$ for the final state of multiple core mergers where $E$ and $M$ refer to the total energy and mass of the system instead of just the core. We revisit this claim in section 4.4 below.

The SP system and consequently the stationary solutions obey a scaling symmetry of the form [Ji and Sin, 1994:

$$
\{t, x, U, \psi, \rho\} \rightarrow\left\{\lambda^{-2} \hat{t}, \lambda^{-1} \hat{x}, \lambda^{2} \hat{U}, \lambda^{2} \hat{\psi}, \lambda^{4} \hat{\rho}\right\}
$$

where $\lambda$ is an arbitrary parameter. Note that $x \propto \rho^{-1 / 4}$ consistent with the relation between the average density of the core and its Jeans length [Hu et al., 2000].

Given a fixed FDM mass, the spherically symmetric soliton is fully characterized by its radius $r_{c}$, which in turn is roughly set by the de Broglie wavelength corresponding to the local velocity in the inner region of the host halo. Since the velocity dispersion varies only mildly within the virial radius of a host halo, the core mass $M_{c} \sim r_{c}^{-1} \sim v_{h} \sim M_{h}^{1 / 3}$ can be directly related to the halo mass $M_{h}$, where $v_{h}$ denotes the virial velocity of the halo. Concretely, it was found that Schive et al., 2014b.

$$
M_{c}=\frac{1}{4} a^{-1 / 2}\left(\frac{\zeta(z)}{\zeta(0)}\right)^{1 / 6}\left(\frac{M_{h}}{M_{\min , 0}}\right)^{1 / 3} M_{\min , 0}
$$


where the factor of four stems from the definition of $M_{c}$,

$$
\zeta(z)=\left[18 \pi^{2}+82\left(\Omega_{m}(z)-1\right)-39\left(\Omega_{m}(z)-1\right)^{2}\right] / \Omega_{m}(z) \simeq 350(180) \quad \text { at } \quad z=0(z \geq 1)
$$

as obtained from numerical simulations of cluster formation [Bryan and Norman, 1998], and

$$
M_{\mathrm{min}, 0}=375^{-1 / 4} 32 \pi \zeta(0)^{1 / 4} \rho_{m 0}\left(H_{0} m / \hbar\right)^{-3 / 2} \Omega_{\mathrm{FDM}}^{-3 / 4} \simeq 4.4 \times 10^{7} m_{22}^{-3 / 2} M_{\odot} .
$$

is the present minimum halo mass below which coherence effects prevent gravitational collapse. For $m_{22}=0.8$ this delays galaxy formation until redshifts $z \lesssim 13$, followed by a potentially enhanced star formation rate within the denser solitonic cores compared to CDM. This fits well to the above discussions on the HMF and UVLF.

The cores admit a scaling relation $M_{c} \sim r_{c}^{-1}$ Guzmán and Ureña-López, 2004]. It was shown by Mocz et al. 2017]; Chan et al. [2018] that this relation extends also into the NFW tail surrounding the soliton in the halo mass range $M_{h} \sim 10^{9}-10^{10} M_{\odot}$. When rescaled, all considered radial halo profiles could be matched, consistently showing that the solitonic core profile transforms into an NFW-like tail when the central density dropped by a factor $\sim$ 100. It was further shown by Chan et al. [2018], that a population of initially stationary stars in the inner halo region can efficiently transport kinetic energy outwards resulting in more compact FDM core configurations and narrower granules. While not yet displaying a realistic scenario, it highlights the need for full FDM simulations including baryons.

Soliton formation has also been studied in depth by Levkov et al. 2018. Within a box of size $L$, they numerically evolve isotropic and homogeneous initial conditions constructed from a superposition of plane waves with Gaussian distributed momenta $\left|\tilde{\psi}_{\tilde{p}}\right|^{2} \sim \exp ^{-\tilde{p}^{2} / \tilde{p}_{0}^{2}}$ with standard deviation $\tilde{p}_{0} \gg \hbar / L$ and random phase $\arg \tilde{\psi}_{\tilde{p}}$. In this kinetic regime, it is found that after time

$$
\tau_{\mathrm{gr}} \simeq \frac{\sqrt{2}}{12 \pi^{3}} \frac{\tilde{p}_{0}^{6}}{m^{5} G^{2} n^{2} \log \left(\tilde{p}_{0} L\right)},
$$

where $n$ is the mean particle density, a solitonic core emerges out of the random fluctuations with growing mass

$$
M_{c}(t) \simeq \tilde{p}_{0}\left(t / \tau_{\text {gr }}-1\right)^{1 / 2} / G m^{2}
$$

and Brownian motion. The formation was equally found for other initial momentum distributions $\left|\tilde{\psi}_{\tilde{p}}\right|^{2} \sim \delta\left(|\tilde{p}|-\tilde{p}_{0}\right)$ and $\left|\tilde{\psi}_{\tilde{p}}\right|^{2} \sim \theta\left(|\tilde{p}|-\tilde{p}_{0}\right)$. Since $M_{c}$ is inversely proportional to the soliton radius, it formally extends over the entire box initially and dynamically shrinks to an increasingly compact object with decreasing radius. The energy spectrum corresponding to the Gaussian distributed initial momenta $F(0, \omega) \sim \omega^{1 / 2} \exp ^{-2 \omega}$ evolves according to the Landau kinetic equation for $t<\tau_{\text {gr }}$ [Zakharov and Karas, 2013]. Around $t \sim \tau_{\mathrm{gr}}$, it becomes thermal, $F\left(\tau_{\mathrm{gr}}, \omega\right) \sim \omega^{-1 / 2} \theta(\omega)$, and starts showing a $\delta$-peak moving from $\omega_{c} \simeq 0$ to increasingly negative $\omega_{c}<0$ over time $t>\tau_{\mathrm{gr}}$. Here, $\omega_{c}$ is the binding 
energy of the soliton resulting in its coherent oscillation $\psi(r, t)=\psi_{c}(r) \exp ^{-i \omega_{c} t}$. Finally, it was numerically proven, that the same nucleation process happens in virializing halos of size $R>\hbar / \tilde{p}_{0}$. Since the soliton formation time $\tau_{\mathrm{gr}}$ is orders of magnitude lower than the free-fall time $\tau_{\mathrm{ff}}$ in these systems, the central solitonic core forms alongside the collapsing halo in agreement with cosmological FDM simulations [Schive et al., 2014a].

\subsection{Self-consistent Construction of FDM Halos}

Even with the adaptively refining mesh used by Schive et al. 2014a], box sizes are limited by the high-resolution demand of FDM simulations. This restricts the simulated halos to masses below $\sim 10^{11} M_{\odot}$ with decreasing statistics of such heavy halos. One possibility to alleviate this problem is the self-consistent construction of isolated virialized FDM halos as done by Lin et al. [2018]. Assuming approximately spherical halo profiles, a shell-averaged gravitational potential $\bar{V}(r)$ can be computed from an initially defined density profile. The wavefunction at time $t_{0}$ is then constructed from the superposition of eigen-functions $\Phi_{n l m}=R_{n l}(r) Y_{l}^{m}(\theta, \phi)$ similar to the hydrogen atom except for the employed non-Coulomb potential $\bar{V}(r)$ :

$$
\psi(t, x)=\sum_{n l m} a_{n l m} \Phi_{n l m}(x) \exp \left[-i E_{n l}\left(t-t_{0}\right)\right]
$$

The complex coefficients $a_{n l m}$ were calibrated using data of five isolated halos with masses $\sim 10^{10} M_{\odot}$ from the cosmological FDM simulation conducted by Schive et al. 2014a. Due to its strongly non-linear dynamics, the solitonic core is not appropriately accounted for by this approach which is meant to provide a good approximation to the almost interactionfree, excited state wavefunction representing the halo. This is a clear sign of the break down of the Schrödinger-Vlasov correspondence in solitonic core formation discussed below. After the construction of the virialized halo the core is manually added using the core-halo mass relation in Equation 3.8. The method was verified by comparing the evolution and stability of the thus constructed halo with its original analog. While the simulated halo has tangentially elongated granules in the outer halo, revealing its dependence on angular momentum, this is not captured in the self-consistent construction resulting in halos with isotropic granules.

Assuming the same functional dependency of the coefficients $a_{n l m}$ with appropriately adopted parameters, a series of self-consistent heavy halos with masses $M_{h}=8 \times 10^{11} M_{\odot}$ could be constructed. They were found to have relatively flat inner halo profiles with radial slopes of $\alpha \simeq-1 / 2$ compared to the hypothesized corona around the core with $\alpha=-5 / 3$ Vicens et al. 2018], or even to the inner slope of the NFW profile decaying radially outward with $\alpha=-1$. 


\subsection{Lagrangian Particle Based Simulations}

Unfortunately, directly solving the SP system with either finite difference or pseudo spectral methods is computationally expensive due to the resolution requirements $\Delta x \sim \hbar / m v$ and $\Delta t \sim \hbar / m v^{2}$. Otherwise, the dynamics of the simulated system is unphysically slowed down. Thus, not only the interference patterns within halos and filaments with large density contrasts have to be highly resolved spatially and temporally, but also low-density voids with large infall velocities towards nearby overdensities.

In order to conduct large-scale cosmological simulations it is therefore imperative to directly evolve the fluid's velocity by utilizing the Madelung transformation yielding the modified Euler Equation 2.22. It is typically discretized using particles that trace the dynamics of the fluid's phase-space density. These Lagrangian N-body particles can then be interpolated onto an Eulerian grid by smoothing them with an appropriately chosen kernel $W(r, h)$, where $r$ defines the distance between the $i$ th particle at $q_{i}$ and cell center at $x_{j}$, and $h$ represents the characteristic kernel width. The density on the grid is then approximated by

$$
\rho\left(x_{j}\right)=\sum_{i} m_{i} W\left(\left|x_{j}-q_{i}\right|, h\right)
$$

Discretizing the underlying Lagrangian before deducing the corresponding Euler Lagrange equation, Veltmaat and Niemeyer [2016] model the contribution to the particles' acceleration $\ddot{q}_{i}$ due to the quantum potential $Q$ by

$$
\ddot{q}=\frac{\hbar^{2}}{2 m^{2}}(\Delta x)^{3} \sum_{j} \frac{\Delta_{n} \sqrt{\rho}}{\sqrt{\rho}} \nabla W\left(\left|x_{j}-q_{i}\right|, h\right),
$$

where $(\Delta x)^{3}$ denotes the cell volume and $\Delta_{n}$ is the discretized Laplace operator with sevenpoint stencil. In contrast to standard smooth particle hydrodynamic approaches the sum is over grid points and not over particle positions [Monaghan, 1992]. This parametrization is explicitly mass and energy conserving and can stably evolve solitonic cores. It therefore lacks only the ability to recover the interference pattern in multi-streaming regions as $Q$ formally diverges in those regions. Instead, the information of the non-vanishing velocity dispersion is directly encoded in the particles' motion. Running cosmological simulations, this approach correctly suppressed small-scale perturbations, while conserving large-scale dynamics and revealing a slight increase in power on scales around the Jeans length.

Since the acceleration depends on the gradient of the artificial kernel $W$ it is not straightforward to generalize the method to variable smoothing widths $h$ without spoiling energy conservation. One possibility could by to construct the density field with dynamically changing kernels but keeping the kernel gradients fixed. Without such an adaptive mesh refinement, the possible grid resolution in cosmological simulations remains limited, rendering it impossible to recover solitonic cores in halos. Slightly different parametrizations were chosen by Mocz and Succi [2015]; Zhang et al. [2018b]; Nori and Baldi [2018]. It remains to be seen if any of them can reproduce solitonic cores and the same degree of energy conservation. 
Building on the above analysis, Armengaud et al. [2017] employed a standard N-body simulation with FDM initial conditions in order to constrain the FDM mass from Ly $\alpha$ data as outlined above. Numerically reconstructing the force due to coherence effects $\nabla Q / m$ from the interpolated density field, they find it to be negligible compared to the gravitational force $\nabla V \gg \nabla Q / m$ outside of regions with collapsed overdensities. In these outer regions, multi-streaming, and the resulting granular structure found in filaments and halos, should not significantly enhance $\nabla Q / m$. Thus, both numerical analyses consistently verify, that standard N-body simulations can correctly reproduce FDM dynamics in low density regions with linear dynamics, while breaking down in overdense regions with nonlinear dynamics and multi-streaming.

We used these results to devise a hybrid method that exploits the efficiency of N-body schemes on large cosmological scales, while using the accuracy of finite difference schemes for a handful of isolated highly resolved halos [Veltmaat et al., 2018. We did so by modifying the publicly available Enzo code [Bryan et al., 2014]. Employing initial conditions generated by Music [Hahn and Abel, 2011] using a transfer function for FDM obtained by AxionCAMB [Hložek et al., 2017], most of the simulation box is evolved with the standard N-body scheme already implemented in Enzo. Isolated halos are then individually selected from low resolution runs and subsequently highly resolved utilizing Enzo's adaptive mesh refinement routines. The FDM wavefunction itself is evolved by a finite difference method only on the highest resolution level. It thus has to be chosen large enough so that coherence effects not captured by the N-body scheme are still negligible at its boundaries, where the wavefunction has to be approximately reconstruct from the N-body particles. They therefore have to carry information about the local value of the wavefunction's complex phase $S(x, t)$. We provide this information by attributing an initial phase $S_{0}$ to each particle. It is obtained by interpolating initial particle velocities $v_{0}$ onto a uniform grid, and solving

$$
\nabla \cdot v_{0}=a^{-1} \nabla^{2} S_{0}
$$

by employing the Poisson solver implemented in Enzo. Equation 3.16 directly follows from Equation 2.18, $S_{0}$ is interpolated back onto the particle positions and evolved in time using the classical wavefunction approach which is applicable if interference effects are negligible - an assumption we made anyways by simulating FDM dynamics with N-body particles. The phase evolution is then governed by the Hamilton-Jacobi equation Trahan and Wyatt, 2005

$$
\frac{\mathrm{d} S_{i}}{\mathrm{~d} t}=\frac{1}{2} v_{i}^{2}-V\left(x_{i}\right)
$$

where $v_{i}$ and $x_{i}$ are the velocity and location of the $i$ th particle, respectively. Again, since the boundary was chosen far enough outside the collapsed halo, we can safely define the wavefunction's amplitude as the square root of the classical density

$$
A(x)=\sqrt{\sum_{i} W\left(x-x_{i}\right)} .
$$


The complex phase is approximated as the argument of the wavefunction obtained by superposing particles weighted by their respective kernels

$$
S(x)=\frac{\hbar}{m} \arg \left[\sum_{i} \sqrt{W\left(x-x_{i}\right)} e^{i\left(S_{i}+v_{i} \cdot a\left(x-x_{i}\right)\right) m / \hbar}\right] .
$$

The velocity dependence is a result of the identification $v_{i}=\nabla S_{i}$, making the above exponent a linearized Taylor series around the particles center of mass $x_{i}$. The underlying theory will be explained in detail below.

The cosmological simulations confirmed solitonic core formation in the central region of virialized halos with NFW-like radial density profiles first found by Schive et al. 2014a. The core-halo mass relation Equation 3.8 could be qualitatively reproduced with a scatter of at most a factor of two. Since only isolated halos without major merger history have been investigated, their core masses are typically underpredicted by Equation 3.8 as the simulated halos have more time to relax towards an equilibrium state. A semi analytic derivation of Equation 3.8 including halo merger histories is presented below. The slight core mass underprediction in isolated halos is also found in the original simulation [Schive et al. 2014a as commented by the same authors in Lin et al. 2018. Lin et al. 2018 suggest to increase the core mass approximated in Equation 3.8 by a factor of 1.7 if isolated halos are considered. This is consistent with core mass increase of the two most strongly growing cores investigated by Veltmaat et al. 2018.

The core was found to have strong quasi-normal excitations failing to relax to the ground state by gravitational cooling on evolutionary time scales. Their frequency

$$
f=10.94\left(\frac{\rho_{c}}{10^{9} \mathrm{M}_{\odot} \mathrm{kpc}^{-3}}\right)^{1 / 2} \mathrm{Gyr}^{-1},
$$

agrees with theoretical investigations by Guzmán and Ureña-López [2004]. These oscillations have been re-investigated by Bošković et al. [2018]. For the first time, Veltmaat et al. [2018] showed that the core is the only gravitationally self-bound virialized object while the granules that constitute the incoherent surrounding halo are unbound. A quantitative investigation of their correlations revealed, that they are spatially coherent on de Broglie wavelength scales $\lambda=h / m v_{\mathrm{vir}}$. The intuitive coherence time scale $t_{c} \simeq h / m v_{\mathrm{vir}}^{2}$ could be numerically confirmed. It is typically short compared to the halos' dynamical time scales, challenging their approximation as quasi particles as suggested by Hui et al. [2017] and applied by Amorisco and Loeb [2018]. Finally, Veltmaat et al. [2018] confirm that the Wigner quasi-probability distribution of the wavefunction matches the classical phase-space distribution function corresponding to the Vlasov-Poisson system when coarse-grained with a Gaussian filter obeying $\sigma_{x} \sigma_{p} \geq \hbar / 2$. For the first time this numerically proves quantitatively the applicability of the Schrödinger-Vlasov correspondance discussed in the next section. As expected for virialized halos their spacially averaged velocity distribution is well fit by a Maxwellian distribution

$$
f(v) d v=\frac{4}{\pi}\left(\frac{3}{2}\right)^{3 / 2} \frac{v^{2}}{v_{\mathrm{rms}}^{3}} \exp \left(-\frac{3}{2} \frac{v^{2}}{v_{\mathrm{rms}}^{2}}\right) d v,
$$


where $v_{\text {rms }} \sim v_{\text {vir }}$ is the root-mean-square velocity [Choi et al., 2014].

\subsection{Schrödinger-Vlasov Correspondence}

With dedicated cosmological FDM simulations [Schive et al., 2014a; Veltmaat and Niemeyer, 2016; Veltmaat et al., 2018 it was shown that FDM and CDM structure formation is indistinguishable on scales above the de Broglie wavelength - expect maybe for the condensating solitonic core in virialized halos. In this section we present the underlying theoretical framework following Uhlemann et al. 2014.

The time evolution of the classical phase-space distribution function $f_{\mathrm{cl}}$ is governed by the Liouville equation ensuring its conservation over time. If effective long-range potentials like the gravitational potential obtained from the Poisson equation are considered, it is referred to as collisionless Boltzmann or Vlasov equation

$$
\partial_{t} f_{\mathrm{cl}}(t, x, p)=-\frac{p}{a^{2} m} \cdot \nabla_{x} f_{\mathrm{cl}}(t, x, p)+m \nabla_{x} V(t, x) \cdot \nabla_{p} f_{\mathrm{cl}}(t, x, p),
$$

which describes collisionless DM without two-body correlations. CDM, being defined as a pressureless fluid with density $\rho_{\mathrm{d}}(x)$ and single valued irrotational momentum $p(t, x)=$ $\nabla_{x} \phi(t, x)$, can be modelled by so-called dust initial conditions

$$
f_{\mathrm{d}}(t, x, p)=\rho_{\mathrm{d}}(t, x) \delta\left(p-\nabla_{x} \phi(t, x)\right) .
$$

Since gravity is a conservative force, it does not allow particle trajectories to cross each other in phase-space, and conserves the irrotationality of the flow. Within this so-called dust model there is initially no velocity dispersion. The fluid thus starts in an absolutely cold condition. Multiple streams only develops after shell-crossing mainly in filaments and virializing halos effectively warming up CDM in those regions. Inserting the first two moments

$$
\begin{aligned}
& M^{(0)}(t)=\int f_{\mathrm{d}}(t, x, p) \mathrm{d}^{3} p=\rho_{\mathrm{d}}(t, x), \\
& M^{(1)}(t)=\int p(t, x) f_{\mathrm{d}}(t, x, p) \mathrm{d}^{3} p=\rho_{\mathrm{d}}(t, x) \nabla_{x} \phi(t, x)
\end{aligned}
$$

into the Vlasov equation then yields the Euler equations of an irrotational fluid

$$
\begin{aligned}
\partial_{t} \rho_{\mathrm{d}}(t, x) & =-\frac{1}{a^{2} m} \nabla_{x} \cdot\left[\rho_{\mathrm{d}}(t, x) p(t, x)\right], \\
\partial_{t} p(t, x) & =-\frac{1}{a^{2} m}\left[p(t, x) \cdot \nabla_{x}\right] p(t, x)-m V(t, x) \\
\nabla_{x} \times p(t, x) & =0 .
\end{aligned}
$$

ensuring density and momentum conservation. Combining these equations, we obtain the Bernoulli equation for a time dependent flow

$$
\partial_{t} \phi(t, x)=-\frac{1}{a^{2} m}\left[\nabla_{x} \phi(t, x)\right]^{2}-m V(t, x) .
$$


Identifying the velocity potential $\phi$ with the classical action $S(t, x)$ as done in Equation 2.18 yields the classical Hamilton-Jacobi Equation 3.17. Comparing the classical Euler equations with Equation 2.19 and Equation 2.21, they only deviate by the quantum potential $Q$, which is second order in $\hbar / m$. It thus vanished in the classical limit $\hbar / m \rightarrow 0$ if density gradients are not diverging. This already points to a close resemblance between CDM and FDM in certain limits and admits the SP approach for fluid dynamics put forward by Spiegel [1980]; Widrow and Kaiser [1993]. These limits can be better quantified when contrasting phase-space distribution functions of FDM and CDM. In order to do so, we need to identify the quantum mechanical density operator $\hat{\rho}=|\psi\rangle\langle\psi|$, assumed to represent a pure state, with an associated phase-space function, typically referred to as a symbol. This can be done via the Wigner transformation yielding the Wigner quasi-probability distribution [Schaller et al., 2014]

$$
\begin{aligned}
f_{W}(t, x, p) & =(2 \pi \hbar)^{-3} \int\langle x-\tilde{x} / 2|\hat{\rho}(t)| x+\tilde{x} / 2\rangle \exp \left[\frac{i}{\hbar} p \cdot \tilde{x}\right] \mathrm{d}^{3} \tilde{x} \\
& =(2 \pi \hbar)^{-3} \int \psi^{*}(t, x-\tilde{x} / 2) \psi(t, x+\tilde{x} / 2) \exp \left[\frac{i}{\hbar} p \cdot \tilde{x}\right] \mathrm{d}^{3} \tilde{x},
\end{aligned}
$$

which is constructed from the wavefunction $\psi$ evolved by the SP system. There are two critical issues here. On the one hand, the distribution strongly oscillates on de Broglie scales preventing it from converging to the classical distribution function in the limit $\hbar \rightarrow 0$. A direct comparison of the time evolution yields [Takahashi, 1989; Schaller et al., 2014]

$$
\partial_{t}\left(f_{\mathrm{W}}-f_{\mathrm{cl}}\right)=\sum_{r \geq 3, \mathrm{odd}} \frac{1}{r !}\left(\frac{\hbar}{2 i}\right)^{r-1} \partial_{x}^{r} V(x) \partial_{p}^{r} f_{W} \sim \hbar^{-1} .
$$

The last identification is a result of the strong oscillations in $f_{W}$. From Equation 3.27 we see that each derivative of the distribution function with respect to its momentum is of order $\hbar^{-1}$. Thus, all terms on the right hand side of Equation 3.28 are of order $\hbar^{-1}$ and $f_{W}$ is ill defined in the classical limit $\hbar \rightarrow 0$. This problem was analytically investigated by Takahashi [1989] and numerically confirmed by Uhlemann et al. [2014]. On the other hand, $f_{W}$ is only a quasi probability distribution as it can have negative values. Both problems can be simultaneously overcome by coarse graining $f_{W}$ with a Gaussian filter Cartwright, 1976; Takahashi, 1989]

$$
f_{\mathrm{FDM}}(t, x, p)=\left(2 \pi \sigma_{x} \sigma_{p}\right)^{-3} \int \exp \left[-\frac{\left(x-x^{\prime}\right)^{2}}{2 \sigma_{x}^{2}}-\frac{\left(p-p^{\prime}\right)^{2}}{2 \sigma_{p}^{2}}\right] f_{W}\left(t, x^{\prime}, p^{\prime}\right) \mathrm{d}^{3} x^{\prime} \mathrm{d}^{3} p^{\prime},
$$

if $\sigma_{x} \sigma_{p} \geq \hbar / 2$. Physically, this corresponds to smoothing over fluctuations on the de Broglie wavelength scale. Alternatively, one can directly use the Husimi representation of the wavefunction itself

$$
\psi_{H}(t, x, p)=(2 \pi \hbar)^{-3 / 2}\left(2 \pi \sigma_{x}^{2}\right)^{3 / 4} \int \exp \left[i p \cdot\left(x-x^{\prime}\right) / \hbar-\frac{\left(x-x^{\prime}\right)^{2}}{4 \sigma_{x}^{2}}\right] \psi\left(t, x^{\prime}\right) \mathrm{d}^{3} x^{\prime},
$$


for which

$$
f_{\mathrm{FDM}}=\left|\psi_{H}\right|^{2} \quad \text { if } \quad \sigma_{x} \sigma_{p}=\hbar / 2
$$

In Equation 3.30, $\psi$ is convoluted with a coherent Gaussian wave packet in order to obtain the smoothed distribution function defined in Equation 3.29. Establishing the Gaussian beam method in the next section, it is shown that this filtering does not erase information stored in the original wavefunction $\psi$.

From Equation 3.27 it can be seen that the time evolution for $f_{W}$ follows exactly the classical $f_{c l}$ if free particles $(V=0)$, uniform densities $(V \sim x)$ or harmonic oscillators $\left(V \sim x^{2}\right)$ are considered. In general, there are additional terms that can be interpreted as quantum corrections or simply higher-order corrections [Schaller et al., 2014]. Apart from numerical discretization errors, the Gaussian beam method presented below can reproduce analytic results exactly, if these corrections are negligible [Kay, 2006]. Conversely, it can be expected to work in regions in which the right-hand side of Equation 3.27 vanishes. It was numerically proven by Veltmaat et al. [2018], that in FDM cosmology simulations this is the case even in collapsed halo with multiple shell-crossings.

A similar smoothing is implicitly employed when the classical distribution $f_{\mathrm{cl}}$ is numerically evolved with N-body schemes, as particles with finite size and discrete momenta are advanced in time. In order to be comparable with Equation 3.29 the numerical smoothing is approximated by

$$
f_{\mathrm{CDM}}(t, x, p)=\left(2 \pi \sigma_{x} \sigma_{p}\right)^{-3} \int \exp \left[-\frac{\left(x-x^{\prime}\right)^{2}}{2 \sigma_{x}^{2}}-\frac{\left(p-p^{\prime}\right)^{2}}{2 \sigma_{p}^{2}}\right] f_{\mathrm{d}}\left(t, x^{\prime}, p^{\prime}\right) \mathrm{d}^{3} x^{\prime} \mathrm{d}^{3} p^{\prime},
$$

where now $\sigma_{x}$ and $\sigma_{p}$ are set by the simulation resolution. Comparing the evolution of the coarse grained distribution functions formally produces the same results as in Equation 3.28 Uhlemann et al., 2014

$$
\partial_{t}\left(f_{\mathrm{FDM}}-f_{\mathrm{CDM}}\right) \simeq \frac{\hbar^{2}}{24} \partial_{x}^{3} V(x) \partial_{p}^{3} f_{\mathrm{FDM}}+\mathcal{O}\left((\hbar)^{4}\right) \sim \hbar^{2}
$$

But now momentum derivatives are of order unity and $f_{\text {FDM }}$ convergence quadratically to $f_{\mathrm{CDM}}$ in the classical limit $\hbar \rightarrow 0$. This quantifies the Schrödinger-Vlasov correspondence. Deviations between CDM and FDM are suppressed by $(\hbar / m)^{2}$ and thus become dominant only in highly oscillatory regions. The correspondance was verified in many cosmological FDM simulations Woo and Chiueh, 2009; Schive et al., 2014a; Veltmaat and Niemeyer, 2016] and quantitatively investigated by Veltmaat et al. 2018].

One aspect is not covered by the Schrödinger-Vlasov correspondence. The formation of bound states with discrete negative energy spectra does not have a classical analog. Levkov et al. 2018] have shown, that the solitonic cores indeed are analogs of quantum mechanical bound states. They are thus macroscopic deviations between CDM and FDM that do not necessarily converge to the classical limit even when coarse grained on the de Broglie scale. 


\subsection{Gaussian Beam Method}

The Gaussian beam method (GBM) was first introduced by Popov [1982]. It allows accurate computations of the wavefunction even around caustics where standard ray tracing methods including the classical wave approximation used by Veltmaat et al. [2018] or the quantum trajectory method [Trahan and Wyatt, 2005] become singular and break down |Ralston, 1982; Engquist and Runborg, 2003|. The main underlying problem is the propagation of point-like entities that are artificially spread out in space by associating them with a kernel function. In contrast, the GBM explicitly evolves spatially extended objects - coherent Gaussian wave packets - that contain non-trivial information also away from there center-of-mass. That way, their superposition correctly reproduces the time evolution of the wavefunction even after shell-crossing. In this section, the GBM is presented following Kay [1994, 2006]. The employed semi-classical approximations were rigorously investigated by Swart and Rousse 2007].

The GBM strongly relies on the fact, that the wavefunction $\langle x \mid \psi\rangle \equiv \psi(x) \in L^{2}\left(\mathbb{R}^{d}, \mathbb{C}\right)$ can be decomposed into coherent Gaussian wave packets

$$
\begin{aligned}
\langle x \mid p, q, \gamma, 0\rangle \equiv & \operatorname{det}\left(\frac{2 \operatorname{Re} \gamma}{\pi}\right)^{1 / 4} \exp [i p \cdot(x-q) / \hbar \\
& \left.-(x-q)^{T} \gamma(x-q)\right]
\end{aligned}
$$

via the Fourier-Bros-Iagolnitzer $(\mathrm{FBI})$ transformation $T_{\gamma}: L^{2}\left(\mathbb{R}^{d}, \mathbb{C}\right) \mapsto L^{2}\left(T^{*} \mathbb{R}^{d}, \mathbb{C}\right)$ :

$$
\begin{aligned}
T_{\gamma}[\psi](q, p) & \equiv \frac{1}{(2 \pi \hbar)^{d / 2}} \int_{\mathbb{R}^{d}}\langle p, q, \gamma, 0 \mid x\rangle\langle x \mid \psi\rangle \mathrm{d} x \\
& \equiv\langle p, q, \gamma, 0 \mid \psi\rangle
\end{aligned}
$$

and its inverse $T_{\gamma}^{*}: L^{2}\left(T^{*} \mathbb{R}^{d}, \mathbb{C}\right) \mapsto L^{2}\left(\mathbb{R}^{d}, \mathbb{C}\right)$ :

$$
T_{\gamma}^{*}[u](x) \equiv \frac{1}{(2 \pi \hbar)^{d / 2}} \int_{T^{*} \mathbb{R}^{d}}\langle x \mid p, q, \gamma, 0\rangle\langle p, q, \gamma, 0 \mid u\rangle \mathrm{d} p \mathrm{~d} q
$$

since Bach, 2002

$$
\langle x \mid \psi\rangle=\left\langle x \mid T_{\gamma}^{*} T_{\gamma} \psi\right\rangle
$$

Given any initial wavefunction $\psi$ we can thus populate phase-space $T^{*} \mathbb{R}^{d}$ with coherent Gaussian beams $\langle x \mid p, q, \gamma, 0\rangle$ weighted by $(2 \pi \hbar)^{-d / 2}\langle p, q, \gamma, 0 \mid \psi\rangle$. Equation 3.37 then implies that we can recover $\psi$ as the integral over all beams. Here, $\gamma \in \mathbb{C}^{n \times n}$ is symmetric and its real part is positive definite. Its imaginary part can be used to extend the Taylor expansion in the initial phase reconstruction to second order. The FBI transformation is identical to the Husimi representation in Equation 3.30. Using the FBI-transformation, it was shown by Kay 1994 that the time integration of $\psi$ can be done by a time integration 
of the beam parameters

$$
\begin{aligned}
\psi(x, t)= & \frac{1}{\left(2 \pi \hbar^{\prime}\right)^{d}} \int_{T^{*} \mathbb{R}^{d}}\left\langle x \mid p_{t}^{\prime}, q_{t}, \gamma_{t}, t\right\rangle \\
& \times \int_{\mathbb{R}^{d}}\left\langle p_{0}^{\prime}, q_{0}, \gamma_{0}, 0 \mid y\right\rangle\left\langle y \mid \psi_{0}\right\rangle \mathrm{d} y \mathrm{~d} p_{0}^{\prime} \mathrm{d} q_{0},
\end{aligned}
$$

with initial conditions $\psi(x, 0) \equiv \psi_{0}$ and time-dependent Gaussian beams

$$
\left\langle x \mid p_{t}^{\prime}, q_{t}, \gamma_{t}, t\right\rangle \equiv C_{p q t} e^{i S_{p q t}^{\prime} / \hbar^{\prime}}\left\langle x \mid p_{t}^{\prime}, q_{t}, \gamma_{t}, 0\right\rangle
$$

Given the classical Hamiltonian function corresponding to Equation 2.15

$$
H^{\prime}\left(p_{t}, q_{t}\right)=\frac{p_{t}^{\prime 2}}{2 a^{2}}+V\left(q_{t}\right)
$$

the beams move on their classical trajectories

$$
\frac{d q_{t}}{d t}=\frac{\partial H^{\prime}}{\partial p_{t}^{\prime}}=\frac{p_{t}^{\prime}}{a^{2}} \quad, \quad \frac{d p_{t}^{\prime}}{d t}=-\frac{\partial H^{\prime}}{\partial q_{t}}=-\frac{d V}{d q_{t}},
$$

where primes denote mass-scaled quantities (e.g. $\left.p^{\prime}=p / m\right)$. We introduce primed quantities in order to avoid an explicit mass dependence in the Schrödinger equation which does not depend separately on $\hbar$ and $m$, but only on their ratio $\hbar^{\prime}$.

As for ray tracing methods, the time evolution of the central phase $S_{p q t}$, being the action of the system, is governed by its Lagrangian $L$ :

$$
S_{p q t}^{\prime}=\int_{0}^{t} L^{\prime} \mathrm{d} t=\int_{0}^{t}\left[\frac{p_{t}^{\prime 2}}{2 a^{2}}-V\left(q_{t}\right)\right] \mathrm{d} t .
$$

The pre-factor $C_{p q t}$ accounts for the time varying Jacobian of the system. It is given by the so-called Herman-Kluk prefactor [Herman and Kluk, 1984]

$$
\begin{aligned}
C_{p q t}= & \operatorname{det}\left(\frac{\pi}{2 \operatorname{Re} \gamma_{t}}\right)^{1 / 4} \operatorname{det}\left(\frac{\pi}{2 \operatorname{Re} \gamma_{0}}\right)^{1 / 4}\left(\frac{1}{2 \pi i \hbar^{\prime}}\right)^{d / 2} \\
& \times \operatorname{det}\left[-\frac{\partial p_{t}^{\prime}}{\partial q_{0}}+2 i \hbar^{\prime} \gamma_{t} \frac{\partial q_{t}}{\partial q_{0}}-2 i \hbar^{\prime}\left(-\frac{\partial p_{t}^{\prime}}{\partial p_{0}^{\prime}}+2 i \hbar^{\prime} \gamma_{t} \frac{\partial q_{t}}{\partial p_{0}^{\prime}}\right) \gamma_{0}^{*}\right]^{1 / 2}
\end{aligned}
$$

with initial Jacobian matrices

$$
J_{0}=\left(\begin{array}{ll}
\partial q_{0} / \partial q_{0} & \partial q_{0} / \partial p_{0}^{\prime} \\
\partial p_{0}^{\prime} / \partial q_{0} & \partial p_{0}^{\prime} / \partial p_{0}^{\prime}
\end{array}\right)=\left(\begin{array}{ll}
\mathbb{1} & 0 \\
0 & \mathbb{1}
\end{array}\right) .
$$

Their time evolution is obtained by differentiating Equation 3.41;

$$
\frac{d J_{t}}{d t}=U J_{t} \quad, \quad U=\left(\begin{array}{cc}
0 & a^{-2} \\
-\frac{d^{2} V}{d q_{t}^{2}} & 0
\end{array}\right) .
$$


The matrix $\gamma_{t}$ can be arbitrarily chosen as long as it fulfills the requirements of $\gamma$, changes continuously with time and stays finite. However, it was found that time-independent $\gamma(t)=\gamma(0)$ yield more accurate results Kay, 1994; Harabati et al., 2004]. We therefore adopt this strategy in the following, although it would be interesting to investigate its ability to adopt to changing grid resolutions in adaptive mesh refinement schemes. Since $\gamma_{t}=\gamma_{0}$ initially, we have $C_{p q 0}=1, S_{p q 0}^{\prime}=0$ and Equation 3.38 reduces to Equation 3.37 as required.

As stated before, the GBM is exact if the considered potential is at most second order, $V(x) \propto x^{2}$ Kay, 2006. The evolution of the wavefunction in inharmonic potentials was found to agree with analytic results only after normalization [Kluk et al. 1986]. Since the normalization of the wavefunction is impractical in FDM cosmology simulations, the GBM breaks down in regions with large inharmonic corrections to the potential. Equation 3.33 shows, that this is the case when the Schrödinger-Vlasov correspondence breaks down as well. Preliminary tests of the GBM already show promising results. They are summarized in chapter 5. In the future, it would also be possible to extend the GBM to higher orders Kay, 2006; Swart and Rousse, 2007]. 


\section{Chapter 4}

\section{Evolution of Solitonic Cores}

This section mostly presents the work published in Schwabe et al. [2016] for which the author of this thesis was mainly responsible for. Initial code development and the final draft was supported by co-authors Jens C. Niemeyer and Jan F. Engels. Dynamics and end states of binary and multimergers of solitonic core were investigated and universal behaviour could be deduced. The results were subsequently utilized in a semi-analytic scheme with which the core-halo mass relation in Equation 3.8 could be reproduced as a consequence of the FDM halos' merger histories. While Xiaolong Du was responsible for the numerical results, the author of this thesis contributed through discussions and assisting in the final draft [Du et al., 2017]. Finally, tidal disruption of solitonic cores, subject to the gravitational field of their host halos, was investigated in Du et al. [2018]. The author of this thesis contributed in the interpretation and presentation of the numerical results obtained from simulations run by Xiaolong Du. The latter papers were additionally co-authored by Jens C. Niemeyer, Christoph Behrens and David Bürger.

\subsection{Numerical Methods}

In Schwabe et al. [2016], we implemented the Schrödinger Equation 2.15into the cosmological hydro code Nyx [Almgren et al. 2013], discretizing the wavefunction $\psi$ on an Eulerian grid. The wavefunction was treated as an additional DM component and integrated using a fourth-order Runge-Kutta solver. Time steps were chosen such that they fulfill the stability constrained of Equation 3.3. We employed the multigrid Gauss-Seidel red-black Poisson solver provided by Nyx to compute the gravitational potential. The cosmological scale factor was always fixed to $a=1$. The FDM mass was set to a fiducial value $m_{22}=2.5$. All simulations were run on grids with $512^{3}$ cells. We monitored the total mass

$$
M[\psi]=\int_{V} \rho \mathrm{d}^{3} x
$$


energy

$$
\begin{aligned}
E[\psi] & =\int_{V}\left[\frac{\hbar^{2}}{2 m^{2}}|\nabla \psi|^{2}+\frac{1}{2} U|\psi|^{2}\right] \mathrm{d}^{3} x \\
& =\int_{V} \frac{\hbar^{2}}{2 m^{2}}(\nabla \sqrt{\rho})^{2} \mathrm{~d}^{3} x+\int_{V} \frac{\rho}{2} v^{2} \mathrm{~d}^{3} x+\int_{V} \frac{\rho}{2} U \mathrm{~d}^{3} x \\
& =K_{\rho}+K_{v}+W
\end{aligned}
$$

and angular momentum

$$
\begin{aligned}
L[\psi] & =\frac{1}{m} \int_{V} \psi^{*}[r \times(-i \hbar) \nabla] \psi \mathrm{d}^{3} x \\
& =\frac{1}{m} \int_{V}\left[r \times \rho \nabla S+\frac{i \hbar}{2} \nabla \times r \rho\right] \mathrm{d}^{3} x \\
& =\int_{V} r \times \rho v \mathrm{~d}^{3} x
\end{aligned}
$$

of the system. In the second line of Equation 4.2, we used the Madelung representation defined in Equation 2.16 and Equation 2.18, whereas in the last line we divided the total energy into gradient energy $K_{\rho}$, kinetic energy $K_{v}$, and potential energy $W$. The third equality in Equation 4.3 is true if the density falls off sufficiently rapidly that boundary terms vanish. While conservation of total mass, energy and angular momentum was verified, each energy contribution was measured separately in order to follow the dynamics of a particular system more closely. We used units $[M]=M_{\odot}$ and $[E]=M_{\odot} \mathrm{km}^{2} \mathrm{~s}^{-2}$. Owing to Equation 3.7, the above defined quantities obey the scaling relations

$$
\left\{M, K_{\rho}, K_{v}, W, L \rightarrow \lambda \hat{M}, \lambda^{3} \hat{K}_{\rho}, \lambda^{3} \hat{K}_{v}, \lambda^{3} \hat{W}, \lambda \hat{L}\right\}
$$

During the relaxation of the system, waves emitted by the merger carry mass and energy toward the numerical boundaries. In order to avoid spurious reheating from reflected waves, we followed Guzmán and Ureña-López 2004] and placed a 'sponge' in the outer regions of the grid by adding an imaginary potential

$$
V(r)=-\frac{i}{2} V_{0}\left\{2+\tanh \left[\left(r-r_{s}\right) / \delta\right]-\tanh \left(r_{s} / \delta\right)\right\} \Theta\left[r-r_{p}\right]
$$

to the Schrödinger equation which efficiently absorbs matter. Here $r$ is the distance from the center of the numerical domain. The Heaviside function $\Theta$ ensures that the non-physical sponge is only added in the outer regions $r>r_{p}$. Let $r_{N}$ be half the box size. We then set $r_{p}=7 / 8 r_{N}, r_{s}=\left(r_{N}+r_{p}\right) / 2, \delta=\left(r_{N}-r_{p}\right)$ and $V_{0}=0.6$. Although our numerical domains were always cubic, we used a spherical sponge since the final states of our simulations are approximately spherically symmetric.

We tested our code by considering a single solitonic core. It was shown by Seidel and Suen [1994; Guzmán and Ureña-López [2004]; Bernal and Guzmán [2006] that it is 
a virialized attractor solution of a broad class of initial conditions. Hence, the core is expected to be stable with low-amplitude excitations caused by numerical errors.

The excitation manifested itself in a periodic variation in the central density. Its amplitude decreased faster than quadratically with resolution implying fast convergence of our code. The central density varied at most on the percent level if $r_{c}$ was resolved by at least 3 cells. For the simulations described below, the typical resolution is greater than 4 cells for all binary mergers and most multiple mergers. The oscillation frequency matches the one found by Guzmán and Ureña-López [2004]. While kinetic and potential energy oscillate with opposite phase, total mass and energy are conserved to better than $10^{-3}$. The oscillation of $\psi$ in the complex plane has the expected frequency Guzmán and Ureña-López, 2004 .

We checked convergences of our code also for binary mergers. Increasing the resolution by a factor of two alters the results only negligibly. In all runs conserved quantities stay constant to better than $10^{-3}$ until matter is absorbed by the sponge.

We used the yt toolkit Turk et al., 2010 for our analysis of numerical data and for the volume rendering of Figure 4.4 and Figure 4.9. Core profiles were fitted employing the radial density profile routines around the density maxima. Although cores with nonvanishing angular momentum are not expected to be perfectly spherical, we find that they can be well fitted by Equation 3.4. Below, we therefore always assume spherical symmetry of the final state.

\subsection{Binary Core Mergers}

We ran a set of binary mergers in order to investigate their dynamics and final states. As a consequence of the scaling relations in Equation 3.7, the initial conditions for an arbitrary binary collision are fully parametrized by few defining parameters, i.e. the relative velocity $v_{\|}$and distance $d$ between the cores, the mass ratio $\mu$ and total mass $M$, the phase difference $\Phi$, and the angular momentum $L_{z}$ perpendicular to the orbital plane chosen to be in the $\mathrm{x}-\mathrm{y}$-plane.

There are two distinct regimes. If the two cores are unbound $(E>0)$ they superpose and pass through each other almost undisturbed Bernal and Guzmán, 2006; González and Guzmán, 2011; Naraschewski et al., 1996, Röhrl et al., 1997], behaving like solitons in this regime. If instead the cores are bound $(E<0)$, they merge rapidly forming a new core [Bernal and Guzmán, 2006]. We found that the mass of the emerging core is largely independent of the initial angular momentum, distance and relative phase, but depends on the ratio of initial core masses and total energy.

In order to analyse the unbound case, we considered two solitonic cores with $\mu \equiv$ $M_{1} / M_{2}=M_{c, 1} / M_{c, 2}=2, L_{z}=0 M_{\odot} \mathrm{Mpc} \mathrm{km} / \mathrm{s}$ and $v_{\|}=4 \mathrm{~km} / \mathrm{s}$. The cores were scaled such that the heavier one had a central density

$$
\rho(0)=1.36 \times 10^{11} M_{\odot} \mathrm{Mpc}^{-3},
$$

roughly corresponding to the present cosmic critical density, giving a core radius $r_{c} \simeq$ 

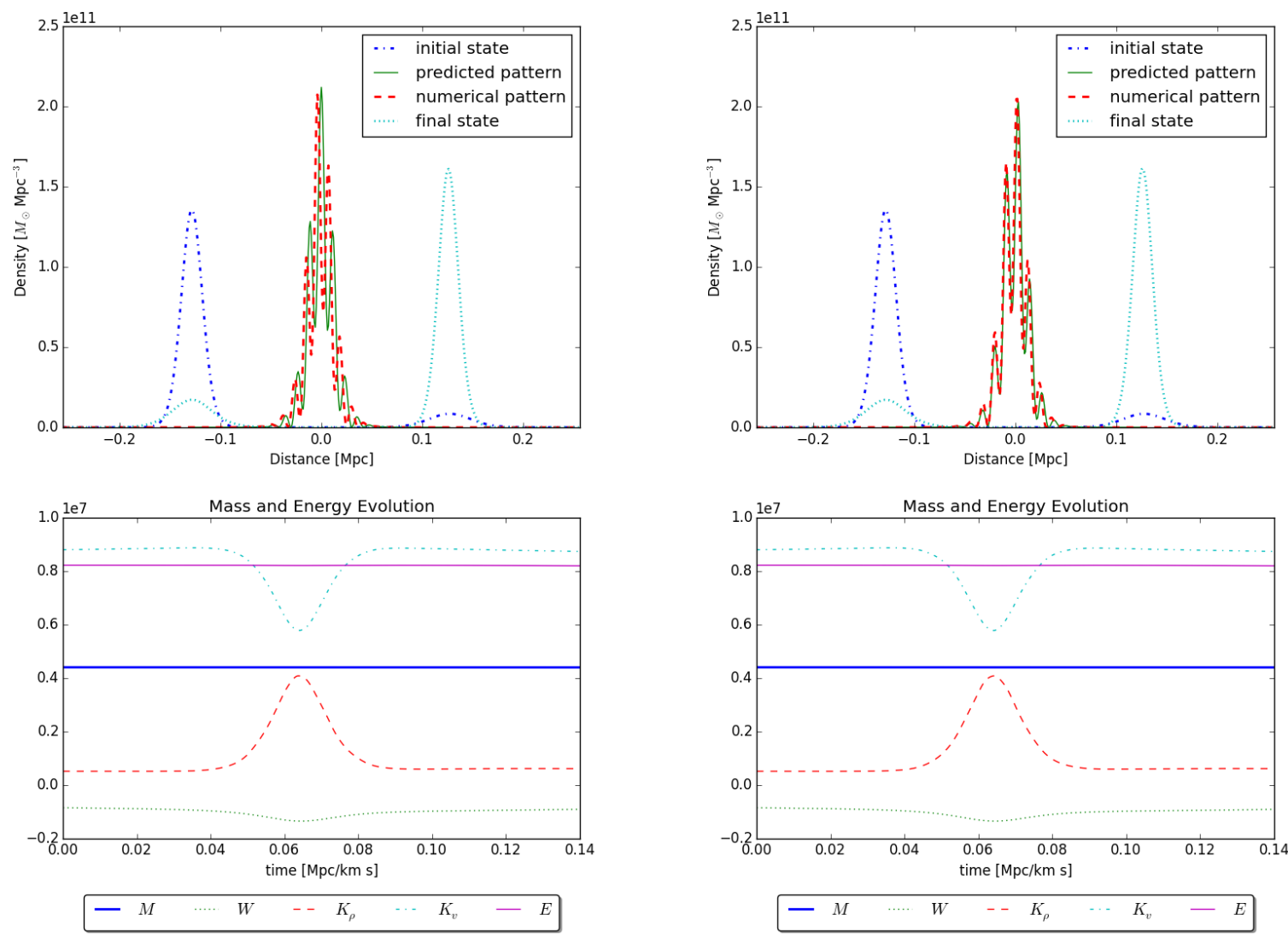

Figure 4.1: Head-on collision of two cores with mass ratio $\mu=2$ and high relative velocity. Upper panels: density profiles at different times for relative phases $\Phi=0$ (left) and $\Phi=\pi$ (right) along the symmetry axis. Numerical results are shown for the initial and final state as well as for the time of maximal interference. For comparison, we plot the interference pattern predicted from Equation 4.7 at the same time. Deviations can be attributed mostly to a small offset in the time of maximal interference. Lower panels: mass and energy contributions. Total energy and mass are conserved, while kinetic energy associated to the cores' relative motion $\left(K_{v}\right)$ is transferred into the interference pattern yielding large values of $K_{\rho}$ during the interaction. The equality of the lower panels shows the independence of the evolution with respect to the initial phase shift $\Phi$. 
$11.6 \mathrm{kpc}$. However, all results are independent of this overall scaling of the problem. The two cores were placed centrally in a $512 \mathrm{kpc}$ cubic box with $d=256 \mathrm{kpc}$ yielding $E \simeq$ $8.2 \times 10^{6} M_{\odot} \mathrm{km}^{2} \mathrm{~s}^{-2}$.

Figure 4.1 shows the density profiles along the symmetry axis and the evolution of global quantities (mass and energy components) for two runs with relative phases $\Phi=0$ and $\Phi=\pi$. The final density distribution as well as the evolution of the global quantities are practically indistinguishable in both cases. Only the interference pattern at the time of superposition depends on the relative phase.

The observed interference pattern follows directly from a superposition of the two solitonic cores. Initially, the cores were placed at $\pm \hat{x}(t=0)= \pm d / 2$. The corresponding wavefunction $\psi(t, x)$ is given by

$$
\begin{aligned}
\psi(t, x)= & A_{1}(|x+\hat{x}|) e^{i(k x / 2+\omega t+\Phi / 2)} \\
& +A_{2}(|x-\hat{x}|) e^{i(-k x / 2+\omega t-\Phi / 2)}
\end{aligned}
$$

where $\left(A_{1}\right)^{2}$ and $\left(A_{2}\right)^{2}$ are the density profiles of the two cores and $k=m v_{\|} / \hbar$ is the wave number corresponding to their relative velocity. The time $t_{\text {int }}$ of maximal interference is defined by $\hat{x}\left(t_{\text {int }}\right)=0$. At that time,

$$
\begin{aligned}
\left|\psi\left(t_{\text {int }}, x\right)\right|^{2}= & A_{1}(|x|)^{2}+A_{2}(|x|)^{2} \\
& \left.+2 A_{1}(|x|) A_{2}(|x|) \cos (k x+\Phi)\right) .
\end{aligned}
$$

The excepted period of the interference pattern is thus given by the de Broglie wave length

$$
\lambda=\frac{2 \pi}{k}=\frac{2 \pi \hbar}{m v_{\|}}
$$

corresponding to the relative velocity. Here, $\lambda \simeq 12 \mathrm{kpc}$. It was therefore well resolved by 12 cells.

The interference pattern predicted by Equation 4.8 matches the numerical results presented in Figure 4.1. During the interaction, gravity slightly contracts the density profiles. Neglecting this small effect, we see that they remain in a superposition state of two solitonic cores even during their interaction. As expected, the potential energy mildly increases during the collision, while mass and total energy are conserved. During the collision, the kinetic energy from the cores' relative motion is stored in the interference pattern, strongly boosting the gradient energy contribution $K_{\rho}$. At later times, the energy is transferred back to the cores' motion. There is no significant decrease in velocity or deformation of the density profiles due to the collision. The cores thus indeed behave like solitons in this regime. This is in contrast to head-on collisions of higher modes resulting in sizable mass exchange during the encounter Guzmán and Avilez, 2018].

The evolution of a bound binary system with negative total energy is very different. In this case, the cores rapidly merge and relax to a new solitonic core by gravitational cooling [Seidel and Suen, 1994; Bernal and Guzmán, 2006]. One interesting exception is the case of binary collisions with perfect phase opposition $\Phi=\pi$ and equal masses $\mu=1$ 

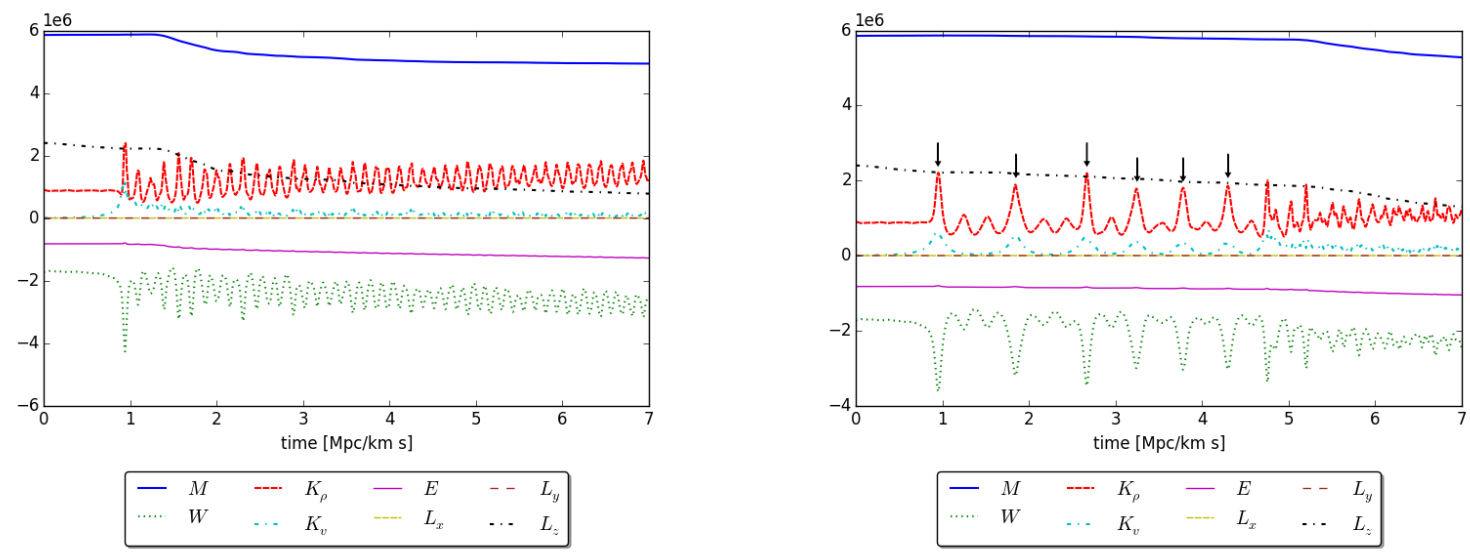

Figure 4.2: Mass, energy and angular momentum evolution of two representative binary collisions with initial values $\mu=1, v_{\|}=0 \mathrm{~km} / \mathrm{s}$, and $L_{z}=2.4 \times 10^{4} M_{\odot} \mathrm{Mpc} \mathrm{km} / \mathrm{s}$ (rescaled by $\left.10^{2}\right)$. Cores with equal phase $(\Phi=0)$ immediately merge (left). In perfect phase opposition $(\Phi=\pi)$, the two cores first mutually repel each other multiple times before merging (right). The bounces are indicated by black arrows. The emerging cores are excited as seen by the oscillations of gradient and gravitational energy, $K_{\rho}$ and $W$. The loss of total mass, energy, and angular momentum results from matter absorption inside the sponge.

during which the destructive interference gives rise to a repulsive effect, causing the cores to bounce off each other [Paredes and Michinel, 2016].

For our study of bound binary collisions, we placed two halos along the central axis in a $1024 \mathrm{kpc}$ cubic box with $d=256 \mathrm{kpc}$. As before, the cores were scaled such that the central density of the heavier core obeyed $\rho(0)=\rho_{\mathrm{cr}}$. Since the two halos emit strongly accelerated mass while merging, a comparatively large box was required such that the mass is able to propagate sufficiently far away from the merger before being absorbed inside the sponge.

In Figure 4.2, we show the mass, energy and angular momentum evolution of two representative runs with $\mu=1, v_{\|}=0 \mathrm{~km} / \mathrm{s}$ and $L_{z}=2.4 \times 10^{4} M_{\odot} \mathrm{Mpc} \mathrm{km} / \mathrm{s}$. On the left, the two cores were in phase. They merged after approximately one free-fall time, $t_{\mathrm{ff}} \simeq 0.94 \mathrm{Mpc} / \mathrm{km} \mathrm{s}$, and formed a new excited solitonic core within roughly one oscillation period. The core's frequency $f \simeq 8 \mathrm{~km} / \mathrm{Mpc} / \mathrm{s}$, implies that it consists of only $70 \%$ of the initial mass Guzmán and Ureña-López, 2004 whereas approximately $30 \%$ of the initial total mass was radiated off by gravitational cooling. This estimate was confirmed by the evolution of the total core mass $M_{c}=M_{c, 1}+M_{c, 2}$ and the total mass $M$ shown in Figure 4.3. Initially, $M_{c} \simeq \frac{1}{4} M$ as expected, decreasing roughly by $30 \%$ during the merger. After a while, the ejected mass reached the sponge and was absorbed. This did not alter the results, since in all conducted runs, the ejected mass is roughly an order of magnitude above the escape velocity $v_{\mathrm{esc}}=\sqrt{2 G M / r}$ and would not have fallen back onto the core.

In the case of solitonic cores with equal mass $(\mu=1)$ but opposite phase $(\Phi=\pi)$, the 


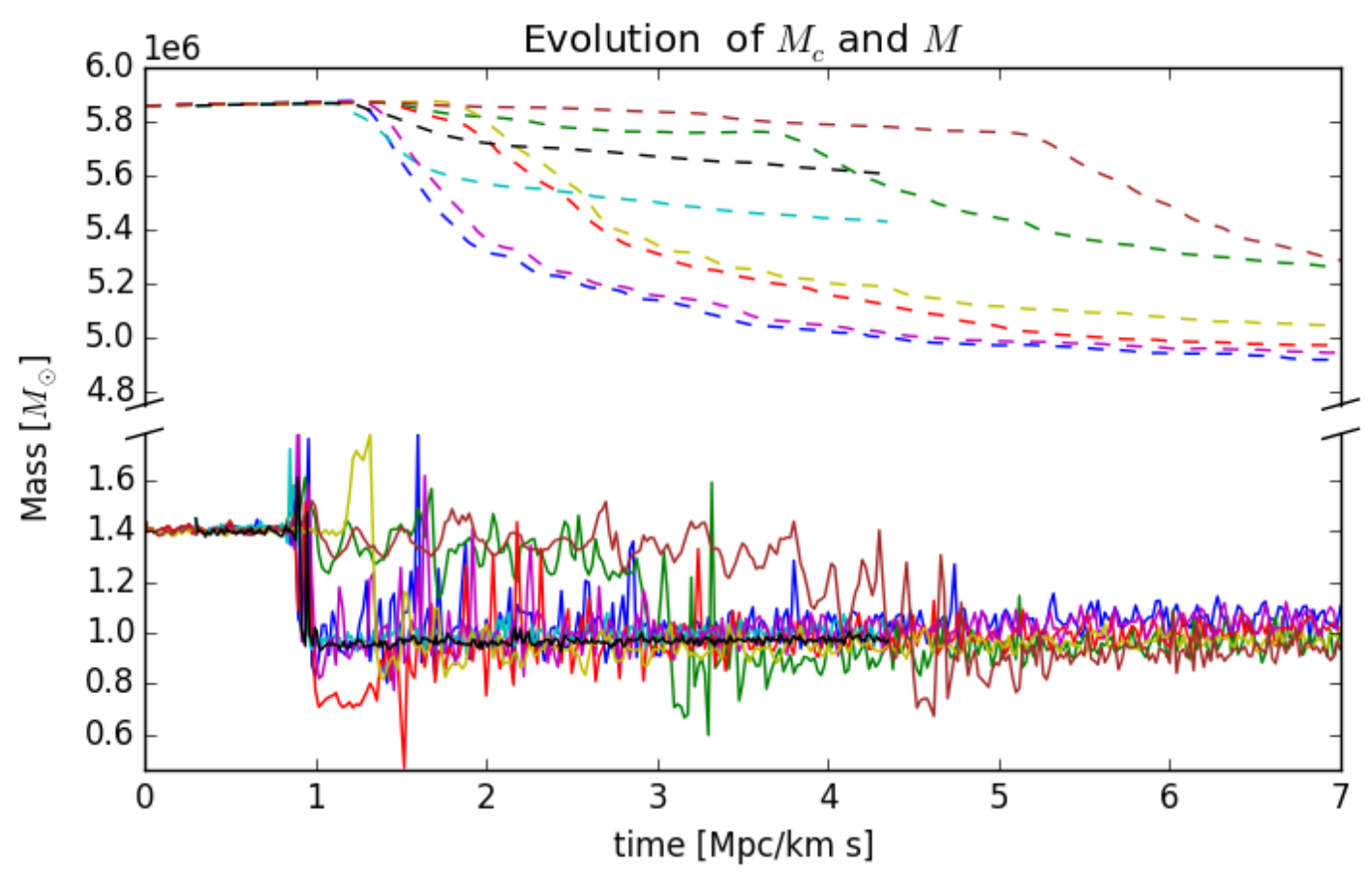

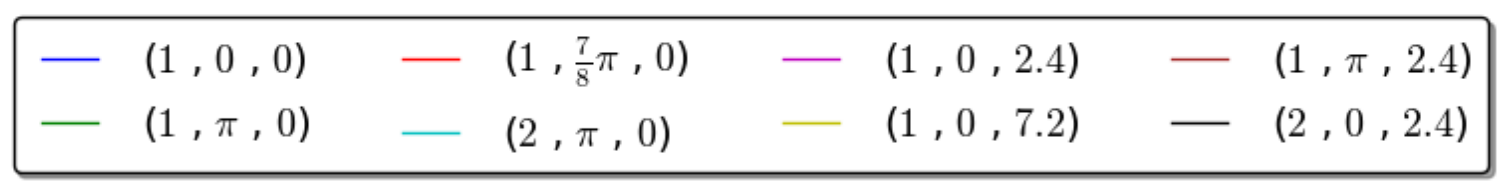

Figure 4.3: Evolution of the core (solid lines) and total (dashed lines) mass for binary mergers. The triplets identify the point $\left(\mu, \Phi, L_{z}\right)$ in parameter space. Angular momentum is given in units of $\left[L_{z}\right]=10^{4} M_{\odot} \mathrm{Mpc} \mathrm{km} / \mathrm{s}$. 

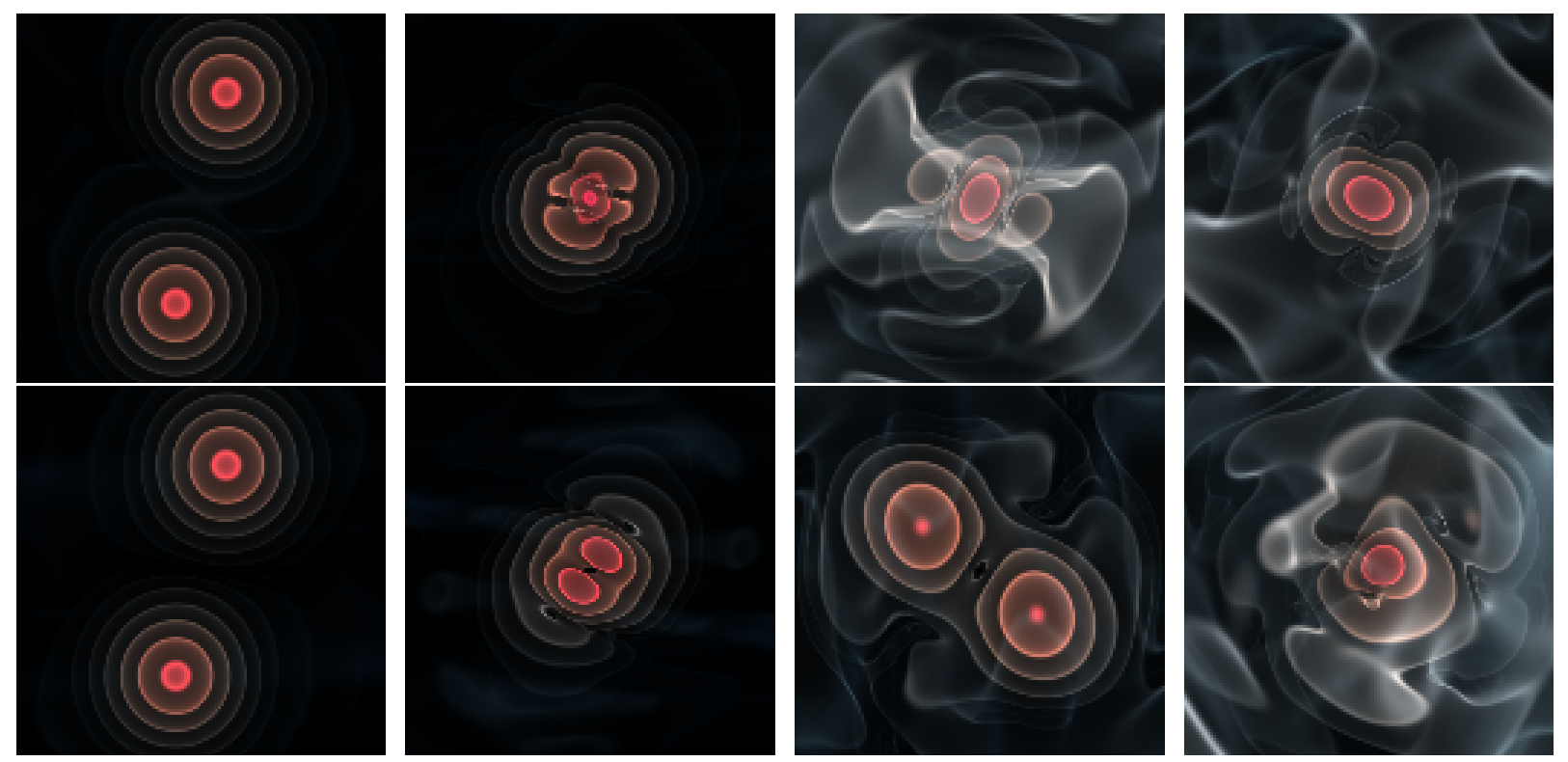

Figure 4.4: Volume rendered images of two representative binary mergers in phase (top) and with opposite phase (bottom) showing the central region of the computational domain at $t=0.7, t=0.94, t=2.0$ and $t=7.0 \mathrm{in} \mathrm{Mpc} / \mathrm{km} \mathrm{s}$.

destructive interference gives rise to a repulsive interaction, causing the cores to bounce off each other several times before merging (cf. right panel of Figure 4.2). This behaviour was also observed by Paredes and Michinel [2016]. The arrows indicate the bounces which result in a noticeable compression of the individual cores. Radiation produced by each encounter results in a damping of the bounces and a decreasing amplitude of the compression. Eventually, the symmetry is broken by the accumulation of small numerical errors producing a slight phase shift, causing the cores to merge in the end. At later times, the evolution is qualitatively identical to the case with $\Phi=0$ as can be seen by comparing the core and halo mass evolution in Figure 4.3 .

Volume rendered images of both runs are shown in Figure 4.4. Especially in the upper panels, a noticeable eccentricity of the newly formed core can be recognized. These rotating ellipsoids are qualitatively those investigated by Rindler-Daller and Shapiro 2009, 2012, 2013, 2014; ; Li et al. 2014]. In particular, their internal velocity fields roughly confine density distributions on elliptical orbits. A slice through a representative ellipsoid is shown in Figure 4.5.

We tested the sensitivity of the repulsive interaction to small deviations from exact phase opposition by considering a phase difference $\Phi=7 / 8 \pi$. In this case, only a single bounce occurs before the cores merge. Similarly, for a mass ratio $\mu=2$ and $\Phi=\pi$ the cores merge without any observable repulsion. These results suggest that in any realistic scenario absent finely tuned phase opposition and mass equality, repulsive behavior of colliding solitonic cores can be ignored for all practical purposes.

We conducted a series of binary mergers spanning the parameter space $\left(\mu, \Phi, L_{z}\right)$. For 


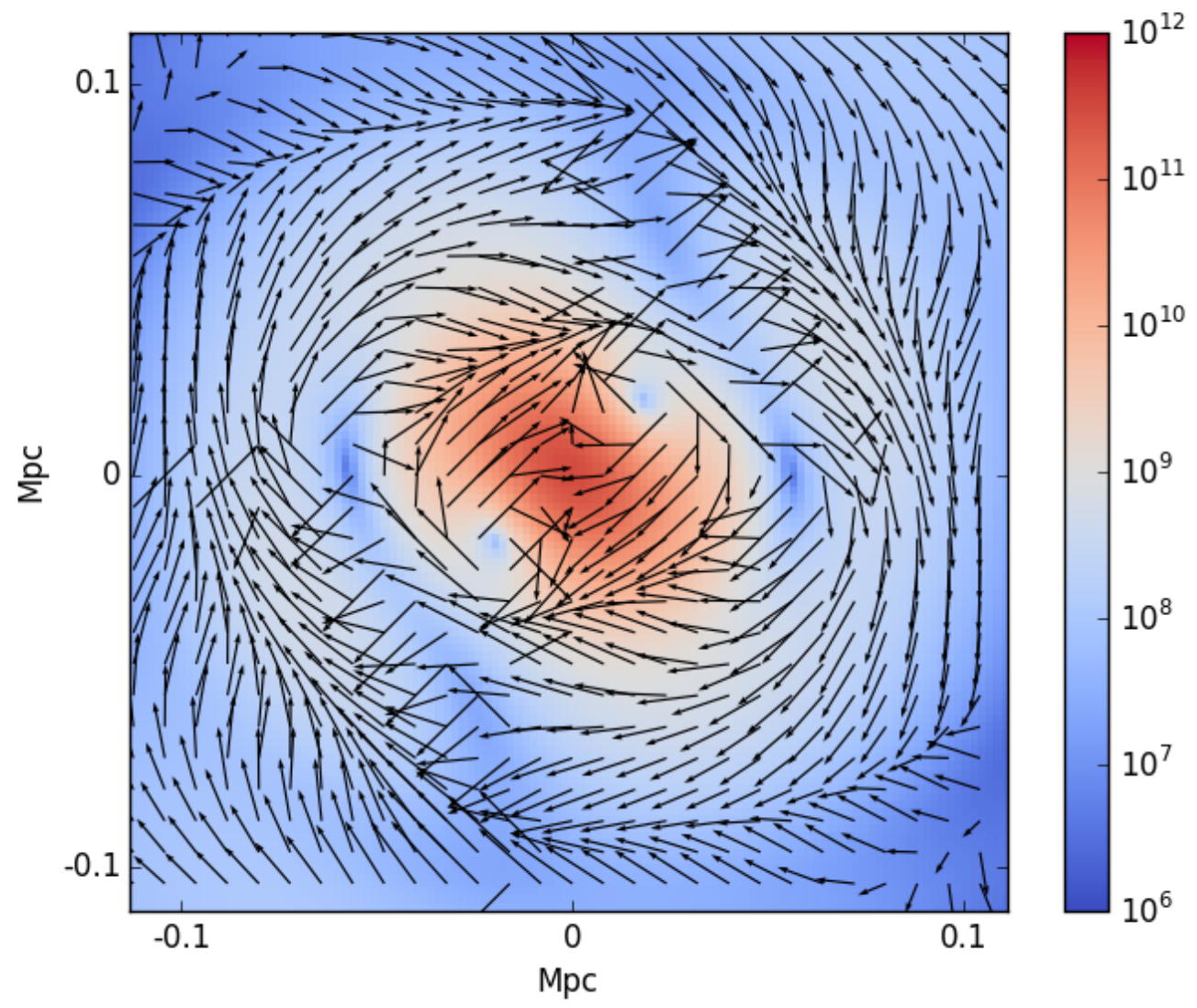

Figure 4.5: Slice through the symmetry plane of a representative ellipsoid. Its density is color-coded while arrows denote the strength and direction of its velocity field. It roughly forms closed elliptical orbits. 

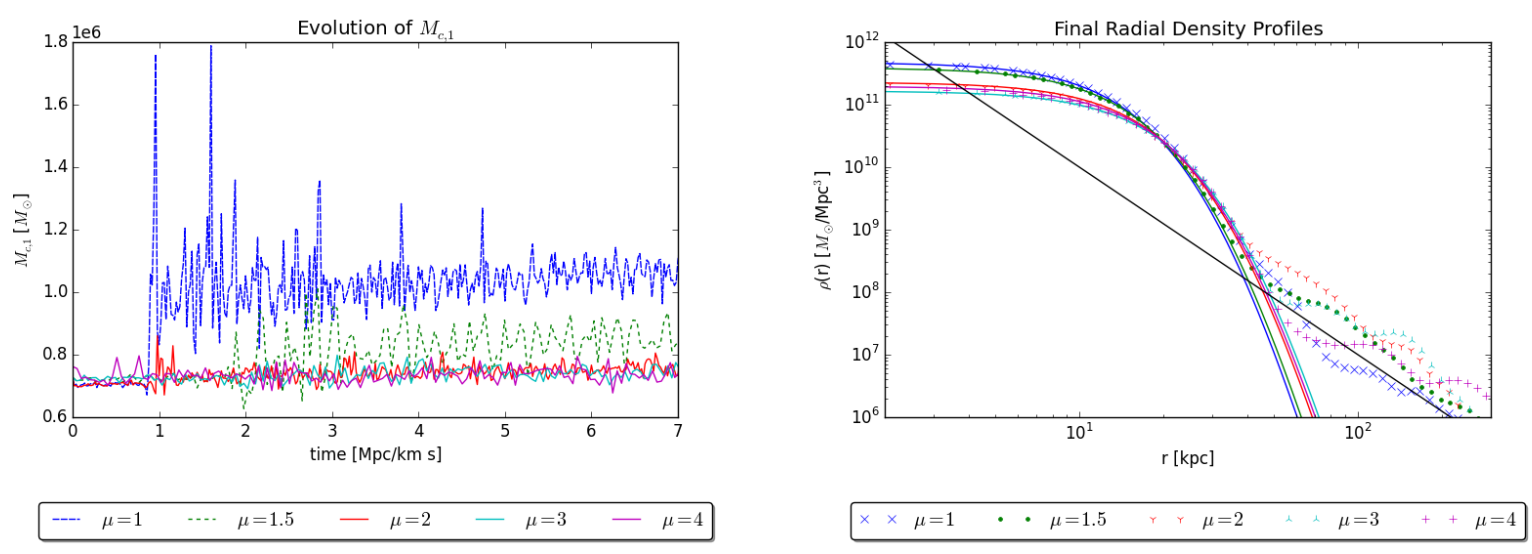

Figure 4.6: Binary mergers with different mass ratios $\mu$. Left: evolution of the core mass of the more massive core. Right: final radial density profiles. Solid lines represent fitted core profiles as defined in Equation 3.4. The black line corresponds to $r^{-3}$ as expected for the outer parts of an NFW profile.

all runs, we set $v_{\|}=0 \mathrm{~km} / \mathrm{s}, \mu \leq 2$, and $L_{z} \leq 7.2 \times 10^{4} M_{\odot} \mathrm{Mpc} \mathrm{km} / \mathrm{s}$ so that the cores are bounded and overlap when reaching the semi-minor axis. Our main result is that the core mass evolution is nearly independent of these parameters within the considered ranges. In all cases, the mass of the emerging core is approximately $70 \%$ of the sum of the progenitors' core masses. The core and total mass evolution of eight representative runs are shown in Figure 4.3. The ratio between final core and total masses is approximately one fifth implying that $80 \%$ of the remaining bound mass resides in the solitonic core while the remainder has formed a diffuse halo around it. Note that due to the restriction to small angular momenta and mass ratios, the total energy varies only very little for all runs. The energy dependent final core masses $M_{c}(E)$ of the above runs are shown in Figure 4.7 (run $1)$.

Assuming a constant fraction of final to initial core masses of $\sim 70 \%$ even for $\mu \neq 1$ implies that the final core is less massive than the more massive progenitor if $\mu \gtrsim 7 / 3$. We therefore expect the change of $M_{c}$ of the more massive core to saturate at roughly this mass ratio. This is qualitatively confirmed by our simulations. For $\mu \gtrsim 2$, the less massive core is completely disrupted and forms a diffuse halo. Figure 4.6 shows the core mass evolution for different mass ratios (left). Here, the initial core mass corresponds to the more massive core. On the right, the final radial density profiles can be seen. They consist of a solitonic core well fitted by Equation 3.4 and a shallow outer tail. Interestingly, the tails in all cases approximately follow a power law decline with a logarithmic slope of roughly -3 as expected for the outer parts of a NFW halo profile. This behavior is consistent with the results of Schive et al. 2014b] but finding NFW-like halos already in the case of binary mergers suggests that it may be more robust than previously expected.

The fitted core masses are mildly energy dependent as can be seen in Figure 4.7 (run 


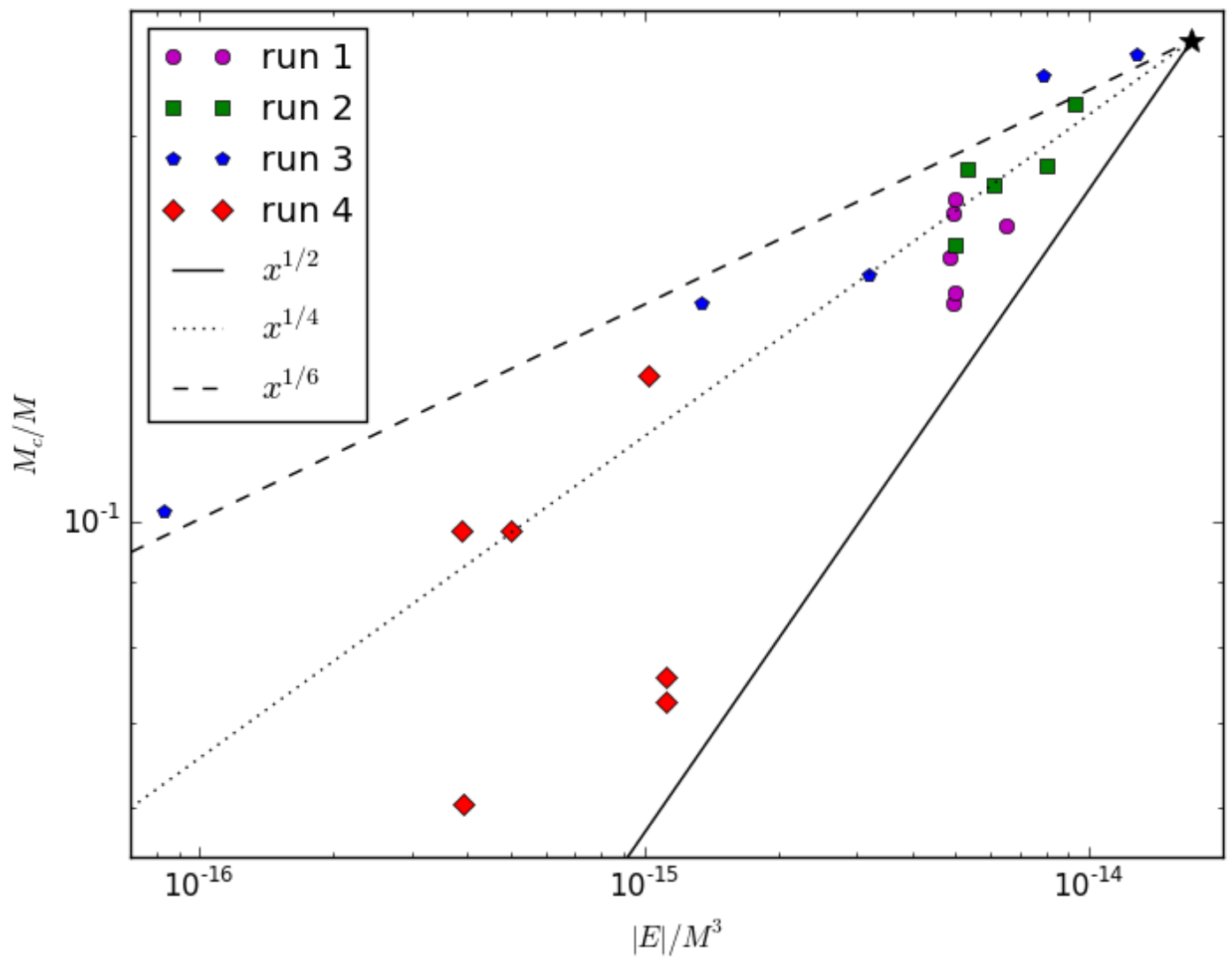

Figure 4.7: Core mass as a function of the total energy and mass. The star indicates the relation for a single solitonic core. Run 1 denotes the simulations with almost equal total energy for different angular momenta and phases. Runs 2 and 3 show the dependence on mass ratio $\mu$ and total energy $E$, respectively. Multiple core mergers are shown as run 4 (cf. section 4.4). See main text for details. 
2). They very broadly follow a power law with

$$
\frac{M_{c}}{M}=656\left(\frac{|E|}{M^{3}}\right)^{1 / 4} M_{\odot}^{1 / 2} \mathrm{~km}^{-1 / 2} \mathrm{~s}^{1 / 2} .
$$

In Figure 4.7, the final core mass $M_{c}$ is normalized to the initial total mass $M$ in order to obtain an invariant relation with respect to the scaling properties given in Equation 3.7. For a single solitonic core, $M_{c} / M \simeq 0.237$ and $|E| / M^{3} \simeq 1.7 \times 10^{-14} M_{\odot}^{-2} \mathrm{~km}^{2} \mathrm{~s}^{-2}$ as indicated by the black star in the upper right corner. This point is consistent with Equation 4.10 since a single core is the limit of infinite mass ratio. A single core is the ground state solution of the SP system. It is therefore the point of minimum energy and maximum core mass per total mass.

Finally, we conducted a series of runs with $\Phi=0, \mu=1, L_{z}=0$ and varying $d$ and $v_{\|}$over a wide range of energies. The fitted final core masses are collectively shown in Figure 4.7 (run 3). The dashed line corresponds to

$$
\frac{M_{c}}{M}=46.7\left(\frac{|E|}{M^{3}}\right)^{1 / 6} M_{\odot}^{1 / 3} \mathrm{~km}^{-1 / 3} \mathrm{~s}^{1 / 3}
$$

indicating a weaker energy dependence for $\mu=1$ than for larger mass ratios.

Our results demonstrate a number of robust features of binary core mergers. Qualitatively, bound systems rapidly merge within roughly one oscillation period of the emerging core after approaching to a distance at which the characteristic core radii overlap. This implies that one of the distinctive features of hierarchical structure formation in FDM cosmologies is the presence of halo and subhalo cores evolving under a sequence of binary mergers which, to very good approximation, can be considered as isolated events. It was shown by Guzmán et al. 2016 that luminous matter cannot follow these extreme dynamics and is expelled from the gravitational potential.

During this dynamical phase, gravitational cooling is most efficient and essentially determines the loss of mass and angular momentum of the merged core, while continuing to dampen its excitations during the ensuing several oscillation periods. One exception is the case of perfect phase opposition and equal masses in which case the cores initially repel each other, leading to a bouncing behavior until small accumulated phase differences again cause a rapid merger on a dynamical time scale. Owing to the fine tuning required for this situation, we do not consider it relevant in the context of cosmology.

The mass of the emerging core does not directly depend on the binary angular momentum, initial distance, and phase shift between the solitonic cores, but only on their mass ratio and total energy. The mass of the more massive core can only be enhanced by binary mergers with mass ratio $\mu<7 / 3$. Otherwise, the smaller core is completely disrupted and forms a NFW-like halo around the more massive one. 


\subsection{Core-Halo Mass Relation}

Subsequently, it was shown by Du et al. 2017] that a mass loss fraction $(1-\beta)=1 / 3$ - close to the one reported on above - can reproduce the numerically found core-halo mass relation given in Equation 3.8. In this study, the modifications to the semi-analytic code Galacticus Benson, 2012; Benson et al. 2012 for FDM described in Du et al. 2016] were employed in order to build 2000 merger trees for root halos with $4 \times 10^{11}<M_{h}<$ $4 \times 10^{13} M_{\odot}$. The mass resolution was set to $2 \times 10^{8} M_{\odot}$. First, the merger history for each root halo was constructed by successively drawing branching events backward in time until the halo mass of the progenitors was below the mass resolution. The branching rate was calculated from the extended Press-Schechter formalism Press and Schechter, 1974, Bond et al., 1991; Lacey and Cole, 1993. Halos which have no progenitors were then evolved forward in time, taking into account different physical effects such as mergers, dynamical friction and tidal stripping. The core mass was traced along the merger history and recalculated at each merger event. It was verified that the final core-halo mass relation is independent of the initial core-halo mass relation for halos close to the minimum cutoff mass in the HMF that form from direct collapse without progenitors. Since there is no other preferred choice, Equation 3.8 was used to set the initial core mass as it is probably most applicable for halos that have just collapsed and not undergone many mergers.

To study the impact of the core mass loss fraction on the final core-halo mass relation, Du et al. 2016 varied $\beta$ between 0.5 and 1.0. Utilizing our above presented simple recipe for the core mass evolution during merger histories, the core mass of the newly formed halo after a major merger with core mass ratio $\mu<\beta /(1-\beta)$ is calculated according to $M_{c}=\beta\left(M_{c 1}+M_{c 2}\right)$, were $M_{c 1}$ and $M_{c 2}$ are the masses of the initial cores. Minor mergers with $\mu>\beta /(1-\beta)$ including smooth accretion do not change $M_{c}$. While for $\beta=0.5$ the core mass is not evolving in time as there are no major mergers, $\beta=1.0$ counts every merger as major, yielding a strongly increasing core mass. The thus semi-analytically obtained final core-halo mass relation was found to be well fitted by the simple scaling power law relation

$$
M_{c}=\frac{1}{4} B\left(\frac{M_{h}}{M_{\min , 0}}\right)^{2 \beta-1} M_{\min , 0}
$$

where the fudge factor $B$ accounts for the slight redshift dependence of the minimum halo mass $M_{\min }(z)$. Thus, $\beta=2 / 3$ reproduces the numerically found core-halo mass relation in Equation 3.8.

\subsection{Mergers of Multiple Cores}

In Schwabe et al. [2016], we also investigated mergers of multiple cores. From our previous analysis we know that the merging time of binaries is negligible with respect to the typical free-fall time. We can therefore safely assume that a multimerger consists of a series of binary mergers within a deeper gravitational well. 
For all runs, we draw halo masses from a Gaussian distribution within the $2 \sigma$-band around a chosen average halo mass. We then place the halos uniformly inside the central numerical domain, rejecting positions that would result in an overlap of halos or close proximity to the outer sponge. Rejected halo positions are redrawn until acceptable. Halos are initialized with random phases. We simulated multimergers of up to 13 halos. As a typical example, Figure 4.9 shows the volume rendered images of a multimerger with 13 halos at three different times.

The final radial density profiles for all runs are presented in Figure 4.8. As in the case of binary mergers and in full agreement with previous studies by Schive et al. [2014b], their central regions can be fitted with a solitonic core profile, Equation 3.4, while the tails fall off like $r^{-3}$ consistent with the outer profile of a NFW-like halo. The final core masses are summarized in Figure 4.7 (run 4). We cannot confirm the $M_{c} \sim(E / M)^{1 / 2}$ scaling shown by Schive et al. [2014b] which may in part be a consequence of the fact that, in contrast with their analysis, all results in Figure 4.7 are normalized to the initial total mass $M$. This eliminates any scaling with energy originating only from the scale invariance of the SP system, making the results more sensitive to the intrinsic energy dependence of multimergers. We verified that this discrepancy is unrelated to the initial phase shifts of individual halos. If the system is initialized with non-zero total angular momentum, we qualitatively recover the rotating ellipsoidal cores studied by Rindler-Daller and Shapiro 2009, 2012, 2013, 2014]; Li et al. 2014].

Our results were largely confirmed by Mocz et al. [2017]. Noticing that the SP system has an additional scaling relation with respect to the FDM mass $m \rightarrow \alpha m$

$$
\{t, x, V, \psi, \rho, M, E, L\} \quad \rightarrow \quad\left\{\alpha \hat{t}, \hat{x}, \alpha^{-2} \hat{V}, \alpha^{-1} \hat{\psi}, \alpha^{-2} \hat{\rho}, \alpha^{-2} \hat{M}, \alpha^{-4} \hat{E}, \alpha^{-3} \hat{L}\right\}
$$

they define the invariant quantity

$$
\Xi=|E| / M^{3} /(G m / \hbar)^{2} .
$$

Simulating a hundred virialized multimergers, they find

$$
\frac{M_{c}}{M} \simeq 2.6 \Xi^{1 / 3}=2.6\left(\frac{|E|}{M^{3}(G m / \hbar)^{2}}\right)^{1 / 3}
$$

over two orders of magnitude in $\Xi$. Note that their definition of $M_{c}$ differs from ours by an additional factor of four. From Equation 3.6 the above relation implies $\left|E_{c}\right| \sim$ $\left|E_{h}\right|$ suggesting that core and halo equilibrate with respect to their energies. Mocz et al. 2017 used periodic boundary conditions. Our slightly smaller core masses are therefore probably due mass and kinetic energy escape at the boundaries or the inclusion of angular momentum. However, our above presented results are broadly consistent with Mocz et al. 2017. After normalizing each final halo to its central density a uniform density profile emerges. The transition between the central solitonic core and outer NFW tail is found to be at $r_{\epsilon}=3.5 r_{c}$ where the density has dropped by roughly two orders of magnitude. Investigating the kinetic energy power spectrum, it was shown that it peaks at $d_{\text {peak }} \sim 2 r_{\epsilon}$, 


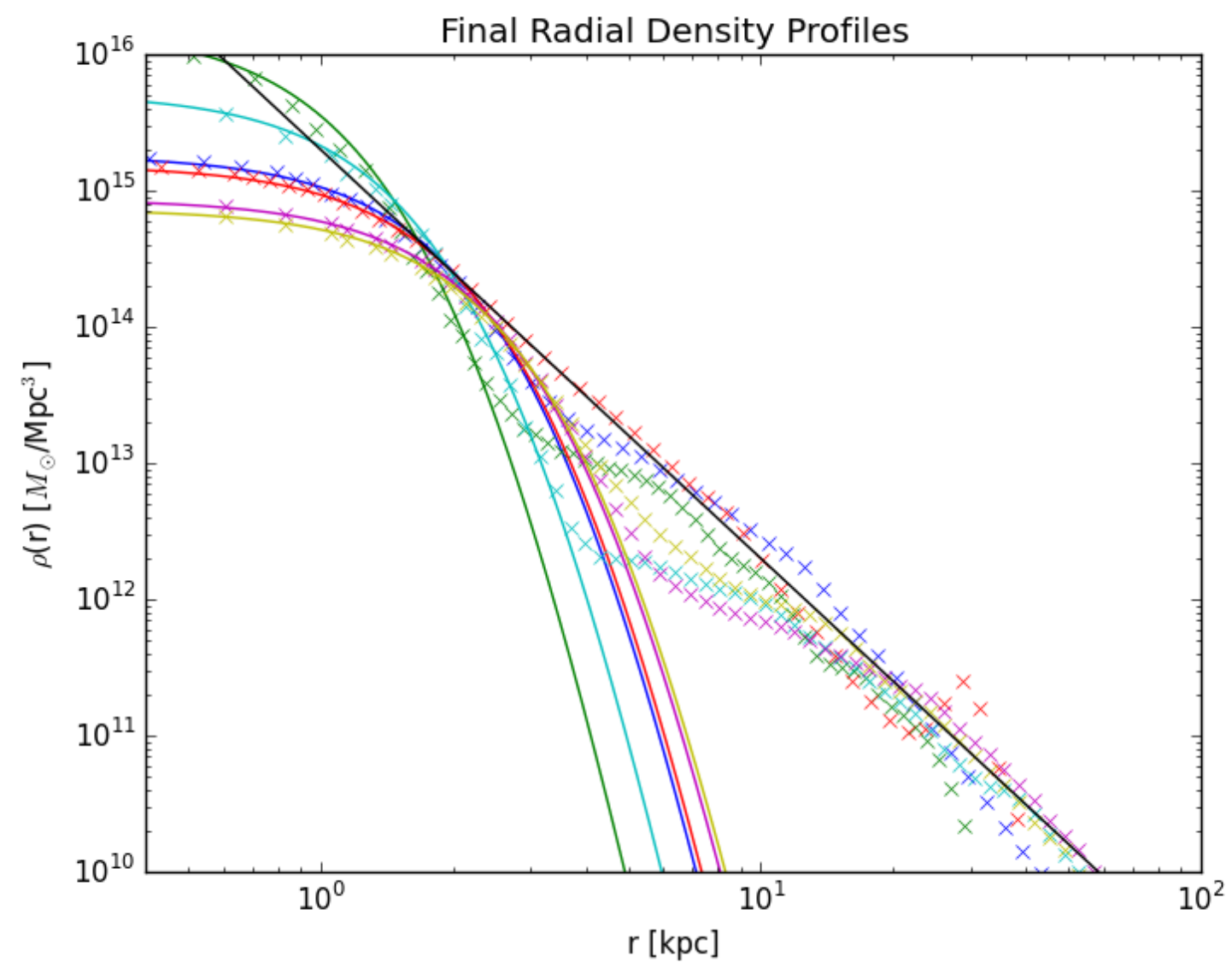

Figure 4.8: Final radial density profiles for all conducted multimerger runs. Solid lines represent fitted core profiles as defined in Equation 3.4. The black line corresponds to $r^{-3}$ as expected for the outer parts of an NFW profile.
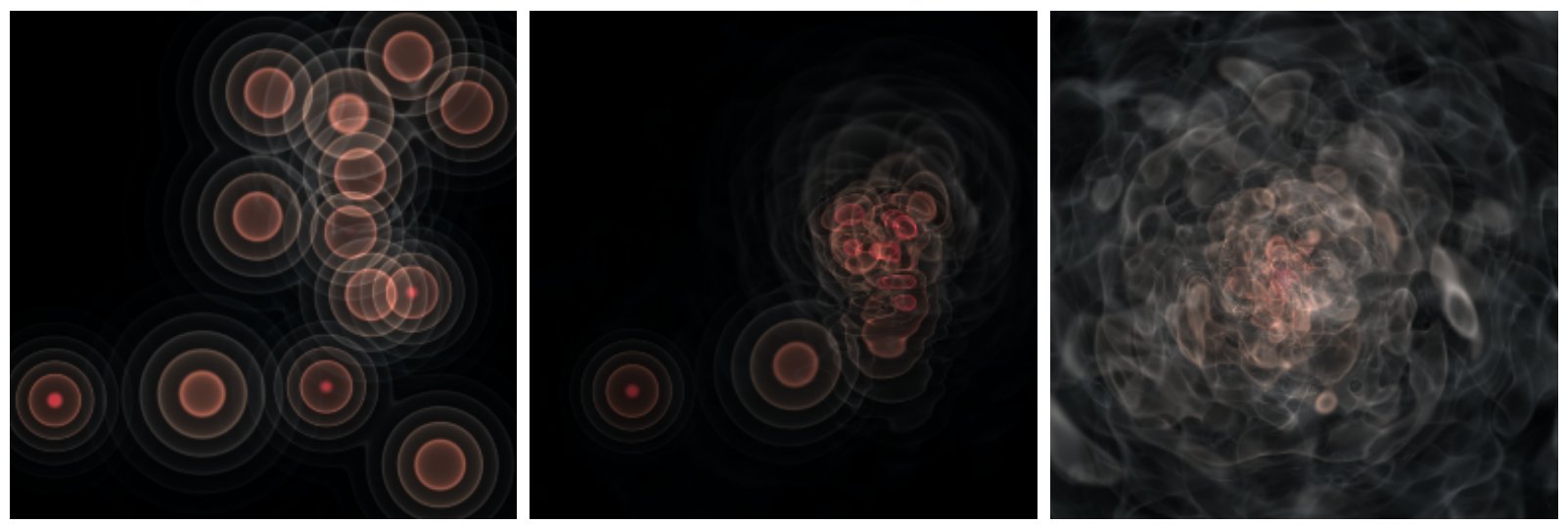

Figure 4.9: Density distribution of a multimerger simulation with 13 halos at different times. 
which is the total width of the soliton within the NFW halo. Plotting the separate radial energy densities of the final virialized haloes, Mocz et al. 2017] find that the core is purely stabilized against gravitational collapse by gradient energy. In the transitional region between $r_{c}<r<r_{\epsilon}$ the classical kinetic energy fraction steeply rises until equipartition is roughly reached in the NFW tail outside $r_{\epsilon}$.

Taking higher modes into account, Vicens et al. 2018] conclude, that the solitonic core should be surrounded by a corona with radial density profile falling as $\rho \propto r^{-5 / 3}$. Unfortunately, the radial density profiles in Figure 4.8 obtained in our simulations strongly fluctuate around the solitonic core making the proposed scaling hard to verify quantitatively. For some final states we see a transient region between the solitonic core profile and the NFW tail with a slope compatible with $\rho \propto r^{-5 / 3}$. This region is not recovered in the averaged density profiles obtained by Mocz et al. [2017], suggesting that it might not be present in fully virialized halos.

\subsection{Tidal Disruption of Subhalo Cores}

Using a fourth-order pseudo spectral code, Du et al. [2018] investigate the tidal disruption of a solitonic core - already stripped off its NFW halo — orbiting around a host halo modelled as a uniform sphere with mass $M_{h}$ well inside the core's circular orbit. The initial ratio $\mu$ between the central density of the soliton $\rho_{c}$ and the average density of the host within the orbital radius is set such that the core radius $r_{c}$ is initially smaller than the tidal radius

$$
r_{t}=\left(\frac{G M_{\mathrm{sat}}\left(<r_{t}\right)}{3 \omega^{2}}\right)^{1 / 3}
$$

where $M_{\text {sat }}$ is the satellite mass enclosed within the tidal radius and $\omega$ is the angular velocity of the satellite. Following Hui et al. [2017], Du et al. [2018] investigate a simple system in which the solitonic core is subject to a spherically symmetric tidal potential $\Phi_{t}(r)=-\gamma \omega^{2} r^{2}$ as a function of distance $r$ to the center of the satellite. Solving the complex eigenvalue problem of the corresponding time-independent SP system, they derive a fitting formula for the satellite's mass loss rate

$$
\frac{\dot{M}_{c}}{M_{c}}=\frac{1}{4} \frac{\dot{\rho}_{c}}{\rho_{c}}=-T_{\text {orbit }}^{-1} \exp \left[a\left(\frac{3}{2 \gamma} \mu\right)^{2}+b\left(\frac{3}{2 \gamma} \mu\right)+c\right],
$$

with the best-fitting parameters $\{a, b, c\}=\left\{5.89794 \times 10^{-5},-8.72733 \times 10^{-2}, 1.6774\right\}$. Here, $T_{\text {orbit }} \equiv 2 \pi / \omega$ is the orbital period. Hui et al. 2017] use $\gamma=\frac{3}{2}$ which includes the effect of the centrifugal force owing to synchronous rotation of the satellite, assuming it to be a rigid body. However, a solitonic core was found to form an irrotational Riemann-S ellipsoid when subject to the tidal force. Therefore, for a solitonic core, $\gamma$ in the tidal potential should be between 1 (without self-rotation) and $\frac{3}{2}$ (with uniform self-rotation that equals the orbital angular velocity). The predicted mass loss rate (4.17) is verified 
numerically. At very early times, the core mass decreases more slowly than the prediction. This can be attributed to the initial conditions. Initially the soliton was set up without self-rotation. The proportionality coefficient in the tidal potential should thus be $\gamma=1$ at the beginning. The core subsequently acquires angular momentum and starts to spin up due to tidal torque, so $\gamma$ approaches $3 / 2$. It was found that the core becomes tidally locked within one orbital period.

One important difference between FDM subhalo cores and rigid-body satellites is that the solitonic core does not sustain uniform self-rotation. Du et al. 2018 found numerically that an initially spherical solitonic core without self-rotation gradually spins up and forms an irrotational Riemann-S ellipsoid in the tidal field of the host, comparable to final states of binary mergers with non-zero angular momentum detailed above. The core is elongated towards the host's center, indicating that the core is tidally locked. However, unlike a rigid body, the core does not rotate uniformly.

As the central density of the core decreases over time, the shape of the ellipsoid also changes. Denoting the semiaxes of the core as $a_{1}, a_{2}$, and $a_{3}\left(a_{1} \geq a_{2} \geq a_{3}\right)$, the change can be characterized by the eccentricities $\epsilon_{i j}=\left[1-\left(a_{j} / a_{i}\right)^{2}\right]^{1 / 2}$ of the ellipsoid. From simulations it is found that $\epsilon_{12} \simeq \epsilon_{13}$, implying that the core is approximately spheroidal.

Assuming constant densities $\rho_{h}$ and $\rho_{\text {sat }}$ for the host and satellite, it was found that the ellipticity of an equilibrated, tidally locked, fluid satellite can be calculated analytically as a function of its density ratio Roche, 1850

$$
\rho_{h} / \rho_{\text {sat }}=\frac{1-\epsilon^{2}}{2 \epsilon^{3}}\left[\left(3-\epsilon^{2}\right) \operatorname{artanh} \epsilon-3 \epsilon\right] .
$$

Since the satellite's density inside the core radius does not change significantly, Du et al. 2018 set $\rho_{h} / \rho_{\text {sat }}=\mu$ and calculated the expected ellipticity from Equation 4.18. They numerically confirmed the approximate solution as long as the core stayed tidally locked.

The rotation of the core can be parameterized by the dimensionless spin parameter as defined in Bullock et al. 2001] for DM halos

$$
\lambda^{\prime}=\frac{L_{c}}{\sqrt{2} M_{c} V R},
$$

where $L_{c}$ is the core angular momentum with respect to its center, $R \equiv\left(a_{1} a_{2} a_{3}\right)^{1 / 3}$ is the mean core radius, and $V$ is the circular velocity at $R$. Since the core's eccentricity increases over time, in order for the core to stay tidally locked, the spin parameter has to increase as well. Thus the core will slightly deviate from tidal locking until it obtains additional angular momentum due to tidal torque and becomes tidally locked again. At late time, angular momentum transfer from orbital motion to self-rotation of the satellite becomes insufficient to maintain tidal locking. The core rotation lags behind its orbital frequency and the core quickly becomes tidally disrupted.

Using [4.17), Du et al. 2018] estimate the survival time of satellite galaxies in the Milky Way. Requiring satellites to survive $N_{\text {sur }}$ orbits translates to a minimum core mass

$$
M_{c}>5.82 \times 10^{8}\left[\mu_{\min }\left(N_{\mathrm{sur}}\right)\right]^{1 / 4} m_{22}^{-3 / 2}\left(\frac{D}{\mathrm{kpc}}\right)^{-3 / 4}\left(\frac{M_{h}}{10^{12} M_{\odot}}\right)^{1 / 4} M_{\odot},
$$


where $D$ denotes the satellite's distance to the center of the host. Assuming the satellite to be disrupted when its core lost $90 \%$ of its mass and taking $\gamma=3 / 2$ and $N_{\text {sur }}=10, \mathrm{Du}$ et al. [2018] find $\mu_{\min }=74$ which is slightly larger than estimated by Hui et al. [2017]. Taking $\gamma=1$ and $N_{\text {sur }}=1$, provides a more conservative constraint $\mu_{\min }=8.4$. Using empirical data of Milky Way satellites from Wolf et al. [2010], Du et al. 2018 conclude the lightest satellites close to the Galactic center will only survive for more than one orbital time if the particle is as heavy as $m_{22} \simeq 20$.

\subsection{Relativistic Axion Stars}

Naively matching the soliton mass and radius in Equation 3.6 to the Schwarzschild radius

$$
r_{s}=2 G M_{s} / c^{2}
$$

of a black hole with mass $M_{s}$ yields a mass scale $M \sim 10^{12} M_{\odot} / m_{22}$ above which FDM solitons are so compact that they should form black holes [Seidel and Suen, 1990; Cotner, 2016]. This is roughly two orders of magnitude above the core masses expected from Equation 3.8 even for the larges halos observed. Nonetheless, if FDM is made out of ultra light axions, attractive self-interactions result in even more compact objects Braaten et al., 2016; Visinelli et al. 2018, that could potentially form BHs. In this regime relativistic corrections have to be taken into account. A review on this more general class of boson stars is given by Liebling and Palenzuela 2017.

The formation and stability of relativist axion stars subject to the standard instanton potential

$$
V(\phi)=m^{2} f_{a}^{2}\left[1-\cos \left(\phi / f_{a}\right)\right]
$$

was investigated in Helfer et al. 2017] using spherically symmetric initial conditions based on solutions for oscillatons in an $\mathrm{m}^{2} \phi^{2}$ potential, for which the full solutions in space and time are known Alcubierre et al., 2003, Ureña-López et al., 2002; Ureña-López, 2002. In a two parameter diagram spanned by initial ADM mass and axion decay constants $f_{a}$, three different regions around a triple point $(M, f) \sim\left(2.4 M_{\mathrm{pl}}^{2} / m, 0.3 M_{\mathrm{pl}}\right)$ could be identified, in which axion stars are either long-lived oscillating axion star solutions, collapse to BHs, or disperse. $\mathrm{BH}$ formation was observed only for large enough initial masses $M>2.8 M_{\mathrm{pl}}^{2} / \mathrm{m}$. For the QCD axion, the typical BH mass formed from axion star collapse is therefore $M_{\mathrm{BH}} \sim 3.4\left(f_{a} / 0.6 M_{\mathrm{pl}}\right)^{1.2} M_{\odot}$. A re-investigation of the phase diagram by Michel and Moss [2018] revealed that boundaries between these region have a complicated diffuse structure. The phase diagram was recovered in Proca star simulations involving vector bosons [Sanchis-Gual et al. 2017.

The stability analysis of axion stars was extended to aspherical initial overdensities by Widdicombe et al. 2018]. They are found to rapidly sphericalize and relax to a final state of collapse that closely follows the known mass-radius relationship for different axion decay constants $f_{a}$. The final state is not fully relaxed as it still shows oscillating perturbations 
similar to the non-relativistic analogs found by Veltmaat et al. [2018]. Applying their stability analysis to a toy model density field with large random fluctuations, Widdicombe et al. 2018] show how field information can be utilized to predict the number densities and masses of axion stars and BHs. They find a mass gap between both species and infer on possible LIGO detection signals. Simulated collapses of overdense regions to compact axion stars show a universal, self-similar behaviour [Levkov et al., 2017].

Binary mergers of two bound boson stars, similar to the non-relativistic case represented above, and their corresponding gravitational wave signal were analyzed by Helfer et al. [2018]; Palenzuela et al. 2017]; Baumann et al. 2018]; Bezares et al. [2017]. Comparable simulations for Proca stars were undertaken by Sanchis-Gual et al. [2018]. In Eby et al. 2017, collisions of axion stars with ordinary stars and neutron stars and the possible subsequent collapse of the axion star were investigated. Recently, potential multi-messenger signals from binary collisions of axion stars with BHs and neutron stars were studied by Clough et al. 2018; Dietrich et al. 2018. 


\section{Chapter 5}

\section{Gaussian Beam Method in Fuzzy Dark Matter Simulations}

This chapter summarizes preliminary results obtained by the author utilizing the GBM presented in section 3.6. The ultimate goal is to use the GBM in FDM simulations as a moderator between low-resolution, large-scale N-body methods in underdense regions well approximated by CDM techniques and the highly resolved, overdense FDM filaments and halos best evolved employing finite difference methods. Ideally, it can be used to extend the classical wavefunction formulation already used in our hybrid simulation method as published in Veltmaat et al. [2018].

The application of the GBM is thereby constrained to regions with intermediate densities. On the one hand, if the density in voids becomes too low, the limited number of beams per unit volume results in an under-sampling of the density field. Insufficient interference between beams then yields unphysical density profiles. In this regime, mass and energy conservation cannot be ensured as both quantities will significantly increase. On the other hand, as outlined in section 3.5, in highly overdense regions strong inharmonic contributions to the gravitational potential result in the break down of the GBM especially around the highly non-linear core region. The exact boundaries between these regimes are yet to be determined. The presented results are to be extended upon completion of this thesis. In all simulations we assumed $m_{22}=2.5$.

\subsection{Implementation of the Gaussian Beam Method}

We implemented the GBM within the publicly available cosmology code Enzo Bryan et al. 2014. The N-body particles were therefore promoted to Gaussian beams by adding attributes $S_{p q t}^{\prime}, A_{0}, C_{p q t}, J_{t}$ and $\gamma_{t}$ to each particle. The new parameters are advanced in time alongside the beam positions and velocities using a fourth-order Runge-Kutta (RK4) algorithm. We added the real and imaginary part of $\psi$ and the corresponding density $\rho$ as further baryonic fields.

In addition to the preexisting timestep criteria enforcing the CFL condition for the 
beams and the cosmological constraint that limits the timestep so that the simulated universe only expands by some fractional amount, we require the change of the beam phases to be at most a fraction $\kappa_{\text {beam }}$ of $2 \pi$ :

$$
\begin{aligned}
& \Delta t_{\text {beam }, \text { kin }}=\min \left(\kappa_{\text {beam }} \frac{4 \pi \hbar^{\prime} a^{2}}{p_{t}^{\prime 2}}\right)_{L}, \\
& \Delta t_{\text {beam }, \text { pot }}=\min \left(\kappa_{\text {beam }} \frac{2 \pi \hbar^{\prime}}{V}\right)_{L},
\end{aligned}
$$

where the potential $V$ is evaluated at the beam centers. As in Bryan et al. 2014 the subscript $L$ emphasizes that the minimum is taken over all beams on a given level $L$. These criteria ensure stability and accuracy of the RK4 method.

A major obstacle in the implementation was an inconsistency in the AMR routines in Enzo that needed to be fixed. Beams on the boundary between two AMR levels with different resolution contribute to the density field of both levels. The density field on the less resolved level is constructed first by interpolating beams from all levels yielding a fully consistent density profile. Subsequently however, the density field on the finer level is incorrectly constructed at the boundaries between levels. Initially, ghost cells are filled with the interpolated density from the lower level. Then, beams from the finer level are added. Thus, lower level particles overlapping with the finer level are not interpolated onto the active region of the density field of the finer level. This results in smaller density values in the active region of the finer levels at the boundary between two levels. Additionally, since ghost cells on the finer level are first filled by interpolating the density field from the lower level, which was constructed using also particles from the finer level, and then particles from the finer level are added on top, particles from the finer level contribute twice within the ghost zones of the finer level. This unphysically enhances density values in ghost cells. Due to the cloud-in-cell method typically used for CDM N-body simulations, the error is restricted to a single layer of cells directly at the boundary. It is therefore negligible. This is not the case anymore within the GBM. Since Gaussian beams span over multiple cells, the density field is incorrectly constructed in a significantly larger region around level boundaries.

The problem could be fixed by interpolating beams from every grid to every other grid independent of their respective levels. This also includes interpolation onto the ghost cells overlapping with neighboring grids. Special care has to be taken since different grid resolutions have to be considered. Unfortunately, this approach requires significantly more communication between processors. This can potentially slow down the algorithm if a high number of grids on many different resolution levels are used.

Additionally, employing large Gaussian shaped kernels for the construction of the real and imaginary part of the wavefunction is computationally much more expensive than the typically used cloud-in-cell method within the usual CDM N-body scheme. For the spherical collapse presented below we therefore constructed the gravitational potential used within the GBM from CDM N-body particles that were evolved alongside the Gaussian beams. This approach re-introduces the inconsistent construction of the CDM density field 
at level boundaries which in turn leads to small discontinuities in the gravitational potential. Below we observe the break down of the GBM in highly resolved overdense regions. It is unclear if this is due to non-negligible inharmonic behaviour in the gravitational potential or its inconsistent reconstruction. In the future, the CDM density field should be constructed with the routines already used for the interpolation of the Gaussian beams. On the contrary, the employed RK4r algorithms with which the beams are evolved in time do not conserve momentum resulting in a slight drift of simulated overdensities. The time integration of the Gaussian beams should therefore be reformulated utilizing the leap frog algorithm already used within Enzo for standard N-body particles.

\subsection{Numerical Tests}

We test our implementation of the GBM by analyzing the analytically calculable time evolution of a Gaussian initial wavefunction

$$
\psi_{0}(x)=\left(\frac{2 \alpha}{\pi}\right)^{d / 4} \exp \left[-\alpha(x-\bar{x})^{2}+i \bar{p}^{\prime}(x-\bar{x}) / \hbar^{\prime}\right] .
$$

The beams are initialized by a Monte Carlo sampling algorithm as described in Kluk et al. 1986. Starting from

$$
\begin{aligned}
& \left(2 \pi \hbar^{\prime}\right)^{-d / 2}\left\langle p_{0}, q_{0}, \gamma_{0}, 0 \mid \psi_{0}\right\rangle A_{0}^{-1} \exp \left[-i S_{0}^{\prime} / \hbar^{\prime}\right]= \\
& {\left[\frac{\sqrt{\alpha \gamma_{0}}}{2 \pi \hbar^{\prime}\left(\alpha+\gamma_{0}\right)}\right]^{d} \exp \left[-\left(\frac{\gamma_{0} \alpha}{\alpha+\gamma_{0}}\left(q_{0}-\bar{x}\right)^{2}-\frac{\left(p_{0}^{\prime}-\bar{p}^{\prime}\right)^{2}}{4\left(\alpha+\gamma_{0}\right) \hbar^{\prime 2}}\right)\right]}
\end{aligned}
$$

with

$$
\begin{aligned}
& A_{0}=\left[\frac{2\left(\alpha+\gamma_{0}\right)}{\sqrt{\alpha \gamma_{0}}}\right]^{d / 2} \\
& S_{0}^{\prime}=\left[\frac{\left(p_{0}^{\prime} \alpha+\bar{p}^{\prime} \gamma_{0}\right)\left(q_{0}-\bar{x}\right)}{\left(\alpha+\gamma_{0}\right)}\right]
\end{aligned}
$$

the right-hand side of Equation 5.4 can be cast into the normalized density

$$
\rho(y, z)=(1 / \pi)^{d} \exp \left[-\left(y^{2}+z^{2}\right)\right]=(1 / \pi)^{d} \exp \left[-r^{2}\right]
$$

with

$$
\begin{aligned}
& y=\sqrt{\gamma_{0} \alpha /\left(\alpha+\gamma_{0}\right)}\left(q_{0}-\bar{x}\right)=r \cos \theta, \\
& z=\left(p_{0}^{\prime}-\bar{p}^{\prime}\right) / \sqrt{4\left(\alpha+\gamma_{0}\right) \hbar^{\prime 2}}=r \sin \theta .
\end{aligned}
$$


Setting

$$
\begin{aligned}
& \eta=\theta /(2 \pi), \\
& \xi=2 \int_{0}^{r} R \exp \left[-R^{2}\right] \mathrm{d} R=1-\exp \left[-r^{2}\right]
\end{aligned}
$$

or rather

$$
\begin{aligned}
& r=\sqrt{-\ln (1-\xi)}, \\
& \theta=2 \pi \eta
\end{aligned}
$$

we see that $y$ and $z$ are distributed according to Equation 5.6 if $\eta$ and $\xi$ are distributed uniformly between zero and one. We can thus initialize an ensemble of $N$ beams by drawing $N$ pairs $\left(\eta_{i}, \xi_{i}\right)$, setting up $\left(p_{0, i}^{\prime}, q_{0, i}\right)$ according to Equation 5.7 and Equation 5.9 and multiplying each beam by $A_{0} \exp \left[i S_{0}^{\prime} / \hbar^{\prime}\right] / N$.

In all test problems $a=1$. We start with $V=0$. The Gaussian wave packet (Equation 5.3 then correctly moves linearly with velocity $\bar{p}^{\prime}$ while spreading with $\alpha_{t}=$ $\alpha_{0} /\left(1+2 i \hbar^{\prime} \alpha_{0} t\right)$. Being the ground state of an harmonic oscillator potential, we additionally checked that the Gaussian wave packet stays constant for $\bar{x}=\bar{p}^{\prime}=0$ and $V=2\left(\hbar^{\prime} \alpha x\right)^{2}$.

In Guzmán and Ureña-López, 2004] spherically symmetric, stationary solutions of the SP system otherwise known as non-relativistic boson stars were investigated. Using dimensionless units in which $\hbar^{\prime}=4 \pi G=1$, their radial amplitude is well fitted by SSchive et al., 2014b

$$
\psi_{\mathrm{bs}}(r)=\psi_{c}\left[1+0.091\left(r / r_{c}\right)^{2}\right]^{-4}
$$

with $\psi_{c}=\lambda^{2}, r_{c}=1.308 / \lambda$, and arbitrary $\lambda \in \mathbb{R}_{+}$. This in turn can be approximated by a Gaussian profile

$$
\psi_{G}(r)=\psi_{c} \exp \left[-\alpha r^{2}\right]
$$

with $\alpha=0.18 \lambda^{2}$. It was shown by Guzmán and Ureña-López [2004], that slightly perturbed boson stars quickly settle into their equilibrium solution $\psi_{\text {bs }}$. We thus expect $\psi_{G}$ to quickly settle into $\psi_{\mathrm{bs}}$ with possibly varying $\lambda$. The collision of two unbound beams produces the correct interference pattern [Schwabe et al., 2016]. Due to non-negligible inharmonic contributions to the gravitational potential of the soliton, it is only stable for a limited time before eventually dissolving, while mass and energy conservation are increasingly violated. This shows the limitation of the approach to harmonic potentials.

\subsection{WKB approximation}

Assuming WKB initial data

$$
\psi_{0}(y) \simeq A_{0}\left(q_{0}\right) \exp \left[i\left(S_{0}^{\prime}\left(q_{0}\right)+\bar{p}^{\prime}\left(q_{0}\right)\left(y-q_{0}\right)\right) / \hbar^{\prime}\right]
$$


with arbitrary reference point $q_{0}$, the beam weights are given by Widrow and Kaiser 1993

$$
\begin{aligned}
\left(2 \pi \hbar^{\prime}\right)^{-d / 2} & \left\langle p_{0}^{\prime}, q_{0}, \gamma_{0}, 0 \mid \psi_{0}\right\rangle \\
& =\operatorname{det}\left(\frac{2 \operatorname{Re} \gamma_{0}}{\pi}\right)^{1 / 4} A_{0} \exp \left[i S_{0}^{\prime}\left(q_{0}\right) / \hbar^{\prime}\right] f\left(p_{0}^{\prime} \mid \bar{p}^{\prime}, \sigma_{p^{\prime}}\right)
\end{aligned}
$$

with normal distribution

$$
\begin{aligned}
f\left(p_{0}^{\prime} \mid \bar{p}^{\prime}, \sigma_{p^{\prime}}\right)= & \operatorname{det}\left(\frac{1}{4 \pi \hbar^{\prime 2} \operatorname{Re} \gamma_{0}}\right)^{1 / 2} \\
& \times \exp \left[-\left(p_{0}^{\prime}-\bar{p}^{\prime}\right) \gamma_{0}^{-1}\left(p_{0}^{\prime}-\bar{p}^{\prime}\right) /\left(4 \hbar^{\prime 2}\right)\right] .
\end{aligned}
$$

Thus, the convolution of $\psi_{0}(y)$ with $\left\langle y \mid p_{0}^{\prime}, q_{0}, \gamma_{0}, 0\right\rangle$ localizes the wavefunction around $q_{0}$ with standard deviation $\sigma_{q}=1 / \sqrt{2 \operatorname{Re} \gamma_{0}}$ (cf. Equation 3.35) and around $\bar{p}^{\prime}$ with $\sigma_{p^{\prime}}=$ $\hbar^{\prime} \sqrt{2 \operatorname{Re} \gamma_{0}}$. This ensures the uncertainty principle $\sigma_{q} \sigma_{p^{\prime}}=\hbar^{\prime}$. Note that the usual factor of one half enters when squaring the amplitude in order to obtain the density. Equation 5.13 can be obtained by taking the limit of $\alpha$ going to infinity in Equation 5.3.

Since $\gamma_{0}$ is a free parameter, we can formally take it to be zero. In this limit, Equation 5.14 becomes a delta distribution $\delta\left(p_{0}^{\prime}-\bar{p}^{\prime}\left(q_{0}\right)\right)$. It can be used to get rid of the momentum integral in Equation 3.38. Additionally, the term proportional to $\gamma_{0}$ vanishes in Equation 3.43. We thus only need to evolve half of the Jacobian matrix in Equation 3.45.

After time integration of each beam according to Equation 3.41, Equation 3.45, the final wavefunction is recovered by summing over all $N$ simulated beams

$$
\begin{aligned}
\psi(x, t)= & \sum_{\text {beams }} c_{p q t} \exp \left[i \left(s_{p q t}^{\prime}+p_{t}^{\prime} \cdot\left(x-q_{t}\right) / \hbar^{\prime}\right.\right. \\
& \left.-\left(x-q_{t}\right)^{T} \gamma_{t}\left(x-q_{t}\right)\right] \theta\left(x-q_{t}\right)
\end{aligned}
$$

with

$$
c_{p q t}=\frac{A_{0}}{\pi^{3 / 2} N} \operatorname{det}\left[\gamma_{t} \frac{\partial q_{t}}{\partial q_{0}}-\frac{1}{2 i \hbar^{\prime}} \frac{\partial p_{t}^{\prime}}{\partial q_{0}}\right]^{1 / 2}
$$

and

$$
s_{p q t}^{\prime}=S_{0}^{\prime}+S_{p q t}^{\prime} .
$$

Here, $\theta$ denotes a truncation function. We truncate beams at $\theta=4 \sigma_{q}$ in all runs. Equation 5.15 corresponds to the integral operator defined in Equation 67 in Kay [1994] applied to WKB initial wavefunctions.

\subsection{Spherical Collapse}

In order to test the suitability of the GBM in cosmological simulations, we simulated the spherical collapse of an initial overdensity with Gaussian shaped radial profile proportional 
to the one given in Equation 5.3 with $\bar{p}^{\prime}=0$ in a cosmological setup. We therefore set the central overdensity to $\delta \rho_{c}=100, \alpha=6.4 \times 10^{3}$ relative to a box size of $l=1.2 \mathrm{Mpc} / \mathrm{h}$ at redshift $z=4$ and choose cosmological parameters as provided by Planck Collaboration 2018. In order to make the simulation less numerically expensive, we approximate the gravitational potential from the CDM density generated by $10^{7}$ standard N-body particles as already implemented in Enzo. Equally many Gaussian beams are initialized with widths $\gamma_{0}=0.25 \Delta x$, four times smaller than the root grid's cell width $\Delta x$. The N-body and GBM were evolved on a $128^{3}$ root grid with up to five levels of adaptive refinement, while the reference finite difference simulation was run on a $512^{3}$ grid. The initial overdensities for all approaches match the analytic profile at least at the per mill level over five orders of magnitude in density contrast. Slice plots through the central plains are shown in Figure 5.1 at different redshifts for all three numerical methods. It can be seen that initially the results obtained by the GBM and finite difference scheme correspond well to each other. The collapse integrated with the GBM slightly lags behind the one from the finite difference method as most visible in the phase evolution. The two methods start to significantly deviate after the formation of a solitonic core in the center at $z=3.0$. This can be attributed either to the break down of the GBM itself in this regime or the inconsistent gravitational potential — obtained from the CDM N-body density — used within it. The evolution of the density field implied by the N-body approach immediately starts to deviate.

As stated before, employing finite difference techniques on a uni-grid restricts one to small box sizes. We therefore could only simulate overdensities that had already collapsed to high central densities $\delta \rho_{c}=100$ indicating an already strongly non-linear regime. In order to take full advantage of the GBM we subsequently simulated Gaussian overdensities with $\delta \rho_{c}=1$ and $\alpha=1.6 \times 10^{3}$ relative to a box size of $l=20.0 \mathrm{Mpc} / \mathrm{h}$ at redshift $z=100$. The CDM density was sampled by $10^{6} \mathrm{~N}$-body particles and again generates the gravitational potential for all employed methods. $10^{7}$ Gaussian beams with $\gamma_{0}=0.25 \Delta x$ were initialized on a $256^{3}$ root grid with up to five levels of adaptive refinement. Additionally, we initialized $10^{6}$ normally distributed beams with zero initial momentum and phase appropriately normalized to serve as initial conditions for the WKB approximated GBM presented in section 5.3. They are evolved according to Equation 5.15-Equation 5.17. As before, initial overdensities for all approaches are sampled well enough in order to match the analytic profile at least at the per mill level over five orders of magnitude in density contrast.

Slice plots through the central plains of the overdensities at redshift $z=7$ are shown in Figure 5.2 for the different employed Lagrangian methods together with the corresponding radial density profiles. They coincide well in the outer halo while strongly deviating in the core region. Since we averaged over radial density shells, we expect the radial profiles obtained by employing the GBMs to coincide with the N-body density profile by means of the Schrödinger-Vlasov correspondence. It is interesting to see that the GBM with WKB approximation fits the N-body profile significantly better than the standard GBM. We emphasise again that the large deviations in the central halo region can be attributed either to the break down of the GBM itself in this regime or the inconsistent gravitational 

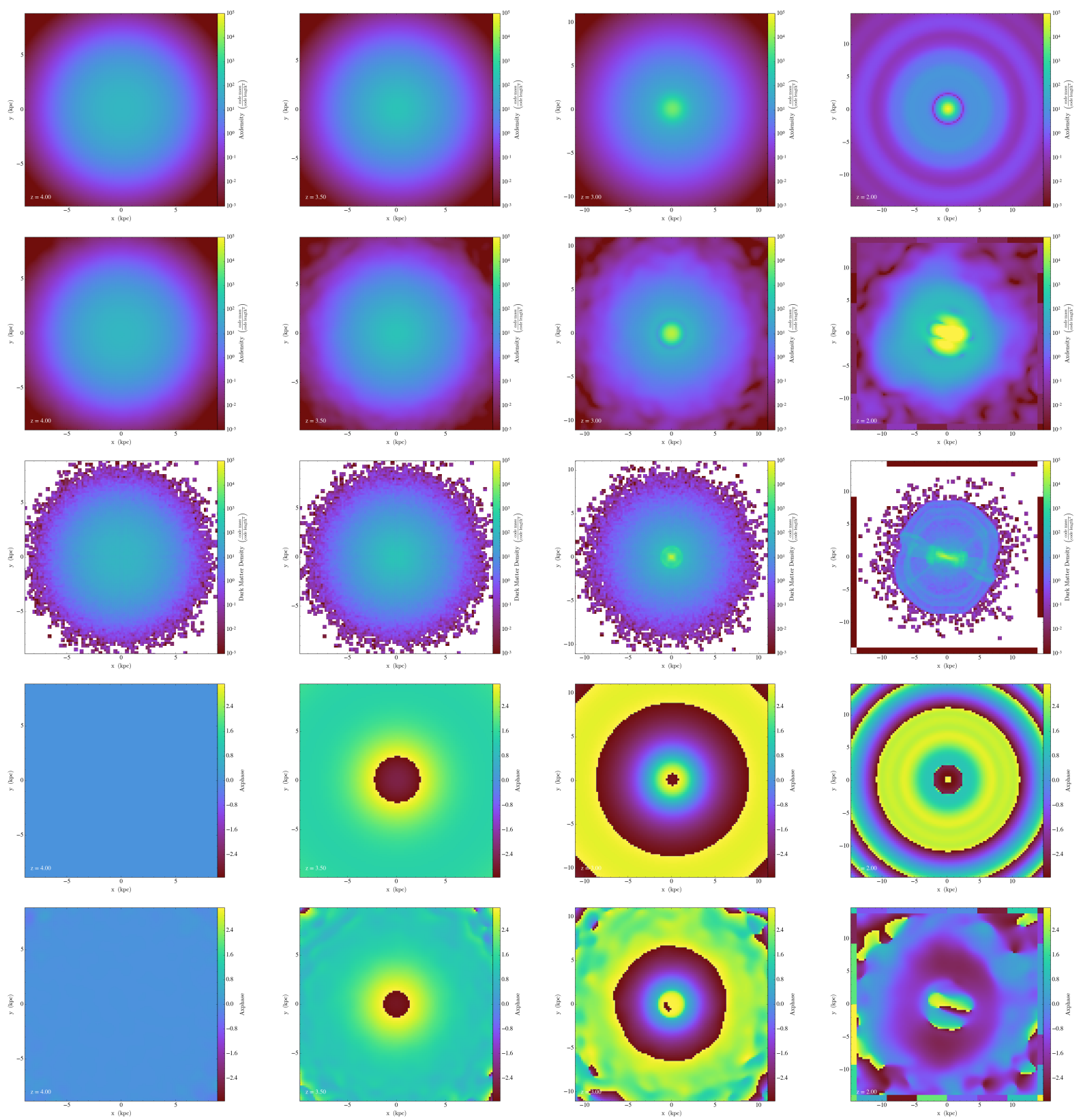

Figure 5.1: Gravitational collapse of a spherical overdensity at different redshifts $z=$ $\{4.0 ; 3.5 ; 3.0 ; 2.0\}$. From top to bottom the density obtained from our (1st row) finite difference, (2nd row) Gaussian beam, and (3rd row) N-body scheme are shown together with the evolution of the corresponding phase integrated within the (4th row) finite difference, and (5th row) Gaussian beam method. It can be seen that the GBM and the finite difference scheme initially yield comparable results. The collapse integrated with the GBM slightly lags behind the one from the finite difference method. The two methods start to significantly deviate after the formation of a solitonic core in the center at $z=3.0$. Density scales are identical in all plots, while phases vary in the interval $[-\pi, \pi]$. 


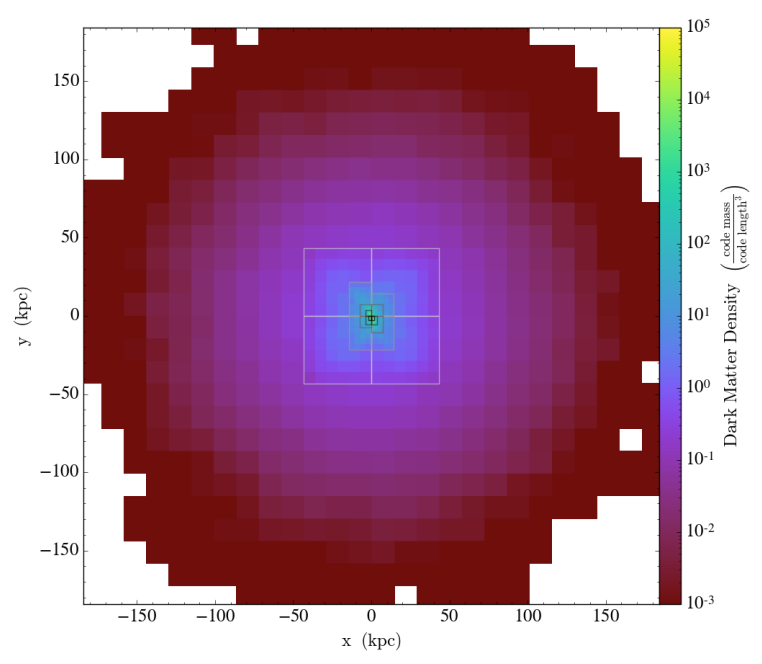

(a) N-body $(n b)$

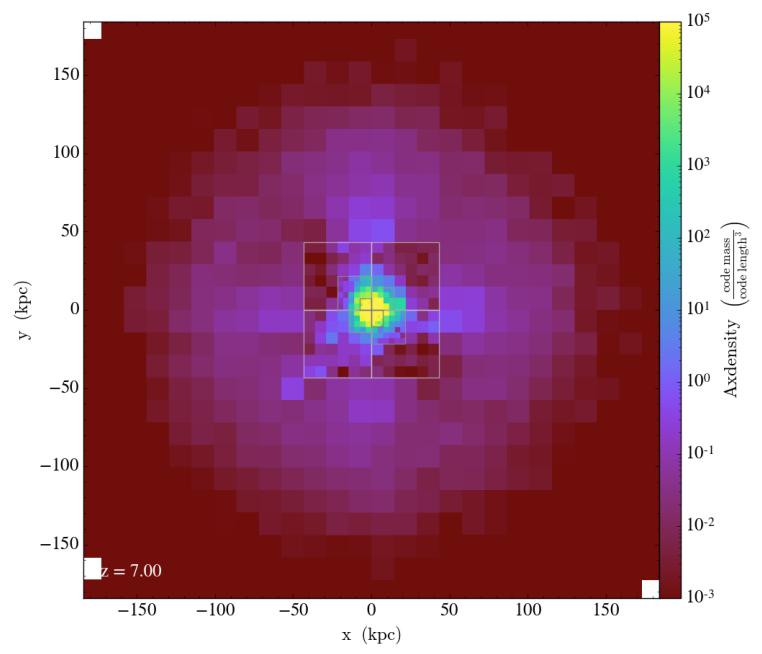

(c) GBM with WBK approximation (gb_wkb)

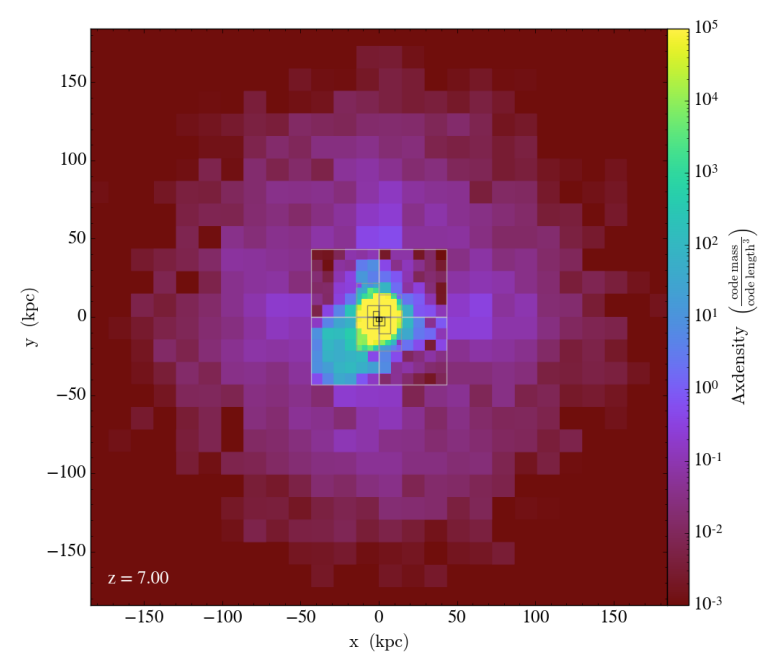

(b) standard GBM (gb)

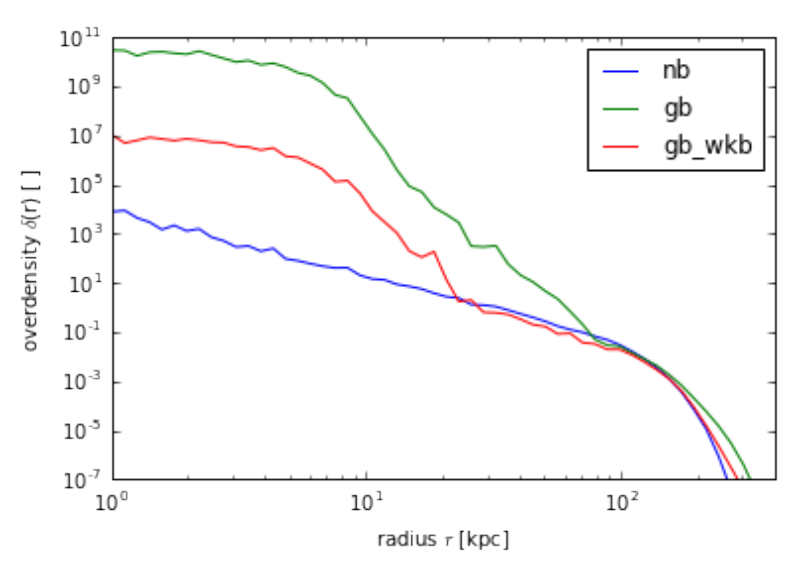

(d) Corresponding radial density profiles

Figure 5.2: Shown are slice plots through the final density profiles after gravitational collapse of a spherical overdensity evolved from redshift $z=100$ to $z=7$ with various numerical methods as indicated by plot labels. The corresponding final radial density profiles are also shown. They match well in the outer halo while strongly deviating in the core region. Since we average over radial density shells, we expect the profiles obtained by employing the GBMs to coincide with the N-body density profile by means of the Schrödinger-Vlasov correspondence. It is interesting to see that the GBM with WKB approximation fits the N-body profile significantly better than the standard GBM. 

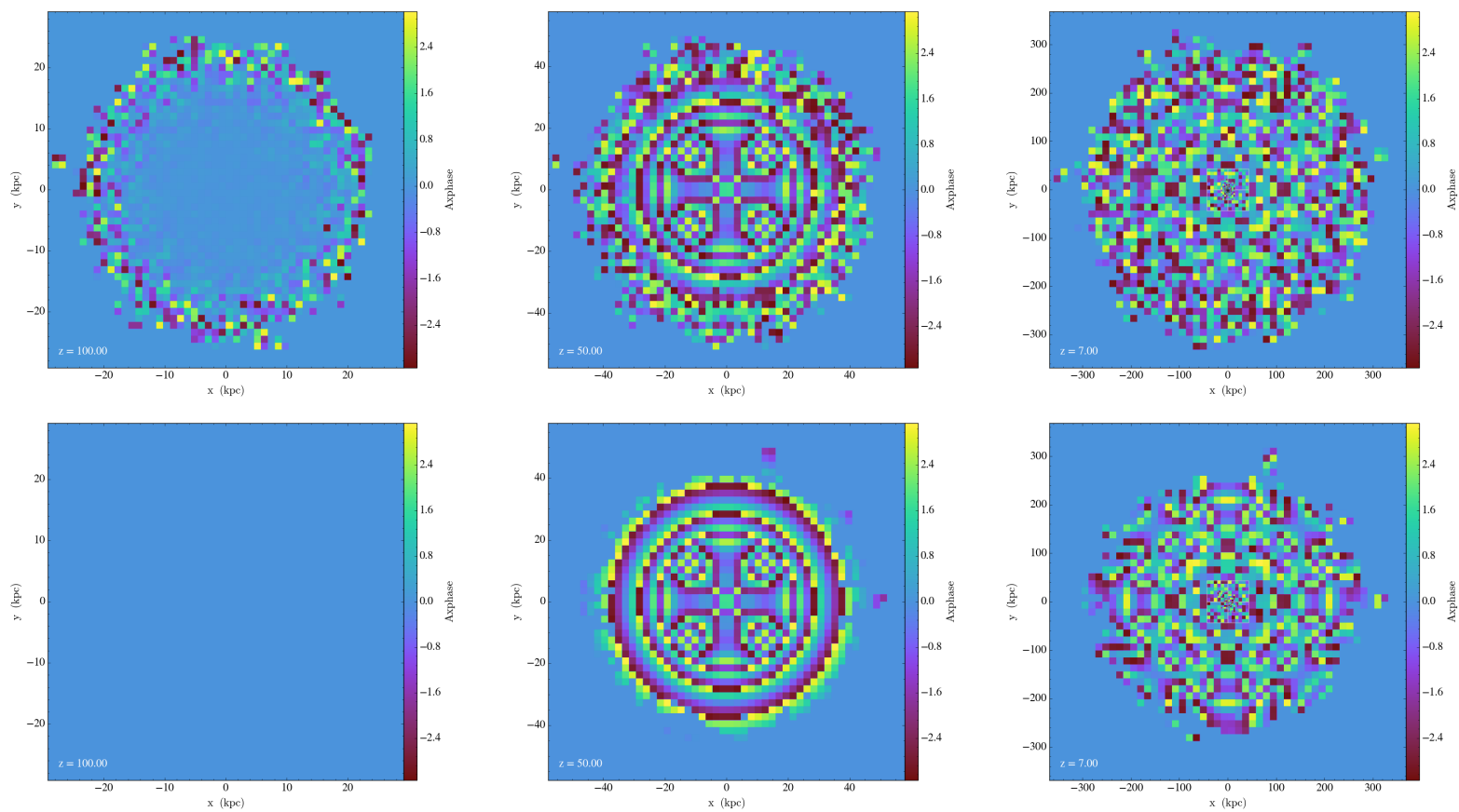

Figure 5.3: Evolution of the complex phase during spherical collapse of a Gaussian overdensity with zero initial momentum at different redshifts $z=\{100,50,7\}$. Top panels show results obtained by the standard GBM, while lower panels were produced with the WKB approximated GBM. The similarity between both phases verifies the correct implementation of both methods and their suitability for the simulation of gravitational collapse. Importantly, the phase is consistently reconstructed by both methods even though it is badly resolved. This has to be contrasted with finite difference approaches needing highly resolved phases. By construction, the initial phase within the WKB approximated GBM is globally zero. Within the standard GBM this is only true in the central regions, where beams overlap enough in order to reproduce the correct phase.

potential. The problem could be alleviated in future simulations by evolving the core region on a highly resolved grid with a finite difference approach.

The evolution of the complex phase during the spherical collapse is shown in Figure 5.3 at different redshifts $z=\{100,50,7\}$. The similarity between the phases obtained by the standard and WKB approximated GBM verifies the correct implementation of both methods and their applicability for the simulation of gravitational collapse. Importantly, the phase is consistently reconstructed by both methods even though it is badly resolved. This has to be contrasted with finite difference approaches needing highly resolved phases.

The already promising results show the enormous potential the GBM has for large cosmological simulations. The WBK approximated GBM is particularly well-suited for those simulations as it is easier to implement and significantly less numerically expensive. The standard approach should be to promote the original N-body particles to Gaussian beams by adding the phase, initial amplitude, beam width and necessary Jacobian matrix 
elements.

On the one hand, since the number of beams per unit volume in underdense regions can be low, their corresponding density profile can not be expected to be correctly modeled by the limited sample of beams. On the other hand, in these linear regions we expect the FDM and CDM densities to coincide well as velocity dispersion should be negligible. The density on the root grid should thus be obtained by the standard N-body cloud-in-cell techniques. On intermediate refinement levels the WKB approximated GBM can then be employed, which can serve as a moderator between the CDM N-body approach and finite difference techniques on the highest refinement levels. In this way, the strengths of all three methods can be combined most effectively. Unfortunately, those cosmological simulations are beyond the scope of this thesis. In the next section we restrict ourselves to the successful implementation of cosmological initial conditions incorporating this new hybrid approach.

\subsection{Cosmological Simulation}

Cosmological initial conditions appropriate for the WKB approximated GBM are constructed using AxionCAMB for an FDM transfer function $T(k)$ [Hložek et al., 2015]. This is then used as an input for MUSIC in order to sample the initial phase-space distribution at redshift $z=300$ within a cosmological box of comoving length $l=50.0 \mathrm{Mpc} / \mathrm{h}$ with $512^{3}$ N-body particles [Hahn and Abel, 2011]. Within Enzo, these particles are interpolated onto Euclidian grids $\left(\rho\left(q_{0}\right), \bar{p}^{\prime}\left(q_{0}\right)\right)$ with $512^{3}$ cells using a Gaussian shaped kernel with width $\sigma_{q}=\Delta q_{0}$. In Figure 5.4, the power spectrum of $\rho\left(q_{0}\right)$ is compared to the input power spectrum $P(k) \sim k^{n_{s}} T(k)^{2}$. As in Veltmaat et al. [2018], the amplitude $A_{0}$ of the initial wavefunction

$$
\psi_{0}\left(q_{0}\right)=A_{0}\left(q_{0}\right) \exp \left[i S_{0}^{\prime}\left(q_{0}\right) / \hbar^{\prime}\right]
$$

is then obtained by taking the square root of the density $\rho\left(q_{0}\right)$. The initial phase $S_{0}^{\prime}\left(q_{0}\right)$ is reconstructed employing Enzo's Poisson solver for integrating

$$
\nabla^{2} S_{0}^{\prime}\left(q_{0}\right)=\nabla \cdot \bar{p}^{\prime}\left(q_{0}\right)
$$

Subsequently, we set up beams on each cell center $q_{0}$ with initial momenta $p_{0}^{\prime}\left(q_{0}\right)=\bar{p}^{\prime}\left(q_{0}\right)$, phases $S_{0}^{\prime}\left(q_{0}\right)$, amplitudes $A_{0}\left(q_{0}\right)$, widths $\sigma_{q}=\Delta q_{0}$, and Jacobian elements $\left(\partial q_{0} / \partial q_{0}=\right.$ $\left.\mathbb{1}, \partial p_{0}^{\prime} / \partial q_{0}=0\right)$. The power spectrum of their corresponding density, reconstructed using Equation 5.15, is also shown in Figure 5.4.

All spectra show the characteristic drop in power at the Jeans scale $k \sim 10 \mathrm{~h} / \mathrm{Mpc}$. While the original spectrum exhibits large oscillations on small scales, they are smoothed over during the construction of the beams due to the convolution with Gaussian kernels. Since the overall shape is in good agreement, we do not consider this a problem. 


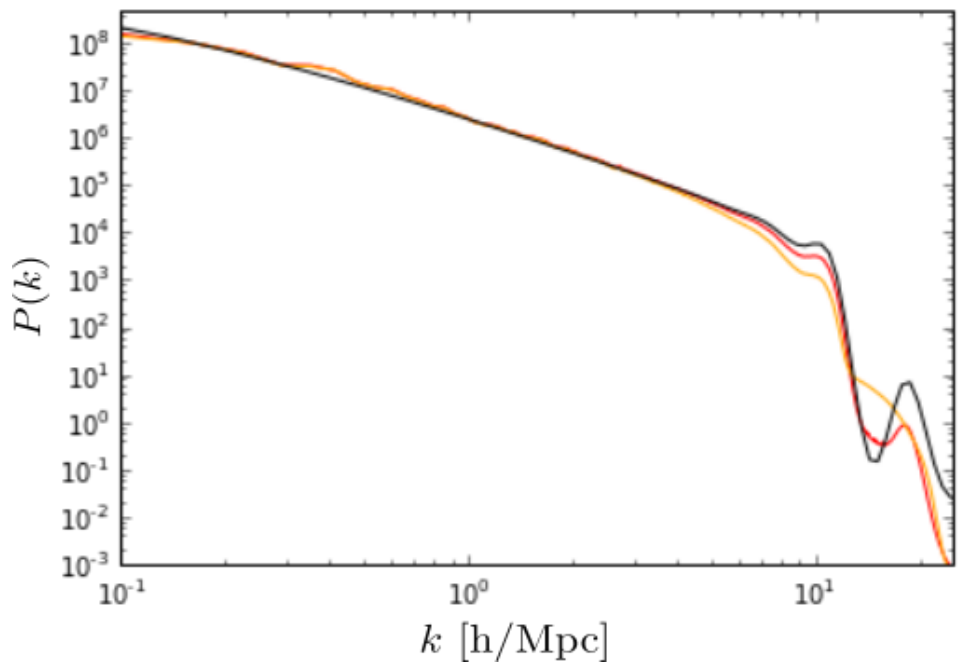

Figure 5.4: Initial power spectra at $z=300$ as obtained from AxionCAMB (black) and within Enzo after wavefunction construction from MUSIC generated phase-space sampling (red) and after reconstruction from Gaussian beams (orange). See text for details. 


\section{Chapter 6}

\section{Conclusion}

Despite being an integral part of the standard model of cosmology, the precise particle nature of dark matter is still unknown. Several well-motivated candidates have emerged over the last couple of decades. Besides the traditional weakly interacting massive particle, originally motivated by the so-called WIMP miracle and arising naturally in supersymmetric extensions of the standard model of particle physics, the QCD axion and axion-like particles have gained increasing attention. In particular, some axions arising from string theories are extremely light. Due to their non-thermal production mechanisms they are nonetheless suitable cold dark matter candidates, if they populate a coherent state effectively represent by a non-relativistic scalar field in a mean field approach. If non-gravitational interactions are suppressed to a degree that renders them negligible for the dynamics of its density evolution, it is commonly referred to as fuzzy dark matter. Its wave-like behaviour on scales below the Jeans scale, formally comparable to the de Broglie wavelength in quantum mechanics, makes it phenomenologically distinguishable from standard pressureless cold dark matter which does not exhibit such a scale. Within fuzzy dark matter cosmologies, gravitational collapse is therefore counteracted and suppressed by the wave-like behaviour of this dark matter candidate on small scales. This can potentially reduce the tension between cold dark matter simulations and observations on galactic scales if particle masses as light as $m=10^{-22} \mathrm{eV}$ are considered. In contrast, lower bounds on the mass can be inferred from their resulting degree of small-scale structure suppression that can be larger than compatible with observations. Currently, fuzzy dark matter research thus concentrates on constraining the boson mass as the single parameter of the theoretical model.

As small scales are the first to become non-linear, the most stringent mass constraints coming, for example, from Ly $\alpha$ measurements, cannot be analytically derived. They instead require dedicated simulations of non-linear structure formation ultimately including baryonic physics as well. In recent years a growing numerical toolbox for such simulations has been developed to which this thesis contributes.

First the author and his collaborators implemented a finite difference method within the preexisting cosmology code Nyx in order to investigate binary mergers of solitonic cores. While unbound binaries showed the expected solitonic behaviour, bound configurations rapidly merged within a single dynamical time scale. The merger history of cores can thus 
be well modelled as a series of binary mergers. The mass of the emerging core was found to dominantly depend on the two progenitor core masses. A simple description could be numerically derived stating that the final core mass is the maximum of the larger progenitor mass and the sum of both initial core masses scaled down by a fraction $\beta=0.7$. Therefore, while smooth accretion and minor mergers with very dissimilar progenitor masses do not result in a mass increase of the heavier core, major mergers with comparable initial core masses yield an increased final mass proportional to the sum of the progenitor masses.

Subsequently, this recipe was implemented into the semi-analytic code Galacticus. It could be verified that the previously found core-halo mass relation can be viewed as a direct consequence of our specified merger history.

Additionally, the author assisted in the investigation of tidal disruption of solitonic cores in the gravitational field of their host halos employing a newly devised spectral code. A fitting formula for the mass loss rate could be derived with which constraints on the fuzzy dark matter mass could be derived via the longevity of Milky Way satellites.

Next, a hybrid cosmology code was implemented within the Enzo framework. It combines the numerical efficiency of standard N-body codes in large underdense regions, where fuzzy and cold dark matter densities are expected to approximately coincide, with the accuracy of finite difference techniques in small overdense filament and halo regions, where interference effects of fuzzy dark matter need to be precisely modelled. The boundary between both regimes was constructed employing the classical wavefunction approach. For the first time, the Schrödinger-Vlasov correspondence could be convincingly shown to hold even in halos outside their core regions. Moreover, the granular interference pattern observed within fuzzy dark matter halos was demonstrated to be unbound, leaving the core to be the only long-living, gravitationally self-bound object within a halo. These results are especially important for semi-analytical halo models and self-consistent halo constructions.

The employed classical wavefunction approach is only valid in the linear regime where gradient energy, comparable to velocity dispersion in standard cold dark matter scenarios, is sub-dominant with respect to gravitational energy. Thus, large regions outside the virial radius of a considered halo have to be modelled with finite difference methods requiring high resolutions in the entire domain in which they are applied in. Pushing the boundary to higher densities increases the numerical error introduced by the classical wavefunction approach.

It can be improved by extending it to the Gaussian beam method which correctly evolves the fuzzy dark matter wavefunction even after shell-crossing as long as inharmonic contributions to the underlying gravitational potential are negligible. The first promising results obtained by implementing the Gaussian beam method in Enzo verify its applicability in highly non-linear cosmic structure formation simulations. This includes a newly devised Gaussian beam method based algorithm utilizing the WKB approximation appropriate for cosmological initial conditions. It is particularly easy to implement in preexisting cosmology code and is significantly less numerically expensive compared to the standard Gaussian beam approach. Full cosmological fuzzy dark matter simulations employing this new technique are beyond the scope of this thesis but will be undertaken by the author in the near future. 
These simulations have the potential to further strengthen the constraints on the fuzzy dark matter particle mass thus paving the way to the possible detection of sub-electronvolt bosons. 


\section{Bibliography}

T. Abel, G. L. Bryan, and M. L. Norman. The Formation of the First Star in the Universe. Science, 295(5552):93-98, November 2001. doi: 10.1126/science.1063991.

M. Abramowitz. Handbook of Mathematical Functions, With Formulas, Graphs, and Mathematical Tables,. Dover Publications, Inc., New York, NY, USA, 1974. ISBN 0486612724.

ACT Collaboration. The Atacama Cosmology Telescope: Temperature and Gravitational Lensing Power Spectrum Measurements from Three Seasons of Data. Journal of Cosmology and Astroparticle Physics, 2014(04):014-014, April 2014. doi: 10.1088/1475-7516/ 2014/04/014.

S. M. K. Alam, J. S. Bullock, and D. H. Weinberg. Dark Matter Properties and Halo Central Densities. The Astrophysical Journal, 572(1):34-40, June 2002. doi: 10.1086/ 340190.

M. Alcubierre, R. Becerril, F. S. Guzmán, T. Matos, D. Núñez, and L. A. Ureña-López. Numerical studies of $\Phi^{2}$-oscillatons. Classical and Quantum Gravity, 20(13):2883-2903, June 2003. doi: 10.1088/0264-9381/20/13/332.

A. S. Almgren, . B. Bell, M. J. Lijewski, Z. Lukić, and E. Van Andel. Nyx: A Massively Parallel AMR Code for Computational Cosmology. The Astrophysical Journal, 765(1): 39, February 2013. doi: 10.1088/0004-637x/765/1/39.

L. Amendola and R. Barbieri. Dark matter from an ultra-light pseudo-Goldstone-boson. Physics Letters B, 642(3):192-196, November 2006. doi: 10.1016/j.physletb.2006.08.069.

N. C. Amorisco and A. Loeb. First constraints on Fuzzy Dark Matter from the dynamics of stellar streams in the Milky Way. arXiv, August 2018. 1808.00464.

E. Armengaud, N. Palanque-Delabrouille, C. Yèche, D. J. E. Marsh, and J. Baur. Constraining the mass of light bosonic dark matter using SDSS Lyman- $\alpha$ forest. Monthly Notices of the Royal Astronomical Society, 471(4):4606-4614, July 2017. doi: 10.1093/mnras/stx1870.

K. S. Arraki, A. Klypin, S. More, and S. Trujillo-Gomez. Effects of baryon removal on the structure of dwarf spheroidal galaxies. Monthly Notices of the Royal Astronomical Society, 438(2):1466-1482, December 2013. doi: 10.1093/mnras/stt2279. 
A. Arvanitaki and S. Dubovsky. Exploring the string axiverse with precision black hole physics. Physical Review D, 83(4), February 2011. doi: 10.1103/physrevd.83.044026.

A. Arvanitaki, S. Dimopoulos, S. Dubovsky, N. Kaloper, and J. March-Russell. String Axiverse. Physical Review D, 81(12), June 2010. doi: 10.1103/physrevd.81.123530.

M. W. Auger, T. Treu, A. S. Bolton, R. Gavazzi, L. V. E. Koopmans, P. J. Marshall, K. Bundy, and L. A. Moustakas. The Sloan Lens ACS Survey. IX. Colors, Lensing, and Stellar Masses of Early-Type Galaxies. The Astrophysical Journal, 705(2):1099-1115, October 2009. doi: 10.1088/0004-637x/705/2/1099.

A. Bach. An Introduction to Semiclassical and Microlocal Analysis. Universitext. Springer New York, 2002. ISBN 9780387953441.

C. A. Baker, D. D. Doyle, P. Geltenbort, K. Green, M. G. D. van der Grinten, P. G. Harris, P. Iaydjiev, S. N. Ivanov, D. J. R. May, J. M. Pendlebury, J. D. Richardson, D. Shiers, and K. F. Smith. Improved experimental limit on the electric dipole moment of the neutron. Physical Review Letters, 97(13), September 2006. doi: 10.1103/physrevlett.97.131801.

N. Banik, A. J. Christopherson, P. Sikivie, and E. M. Todarello. New astrophysical bounds on ultralight axionlike particles. Physical Review D, 95(4), February 2017. doi: 10.1103/ physrevd.95.043542.

R. Barkana and A. Loeb. Detecting the earliest galaxies through two new sources of 21 centimeter fluctuations. The Astrophysical Journal, 626(1):1-11, June 2005. doi: $10.1086 / 429954$.

M. Bartelmann and P. Schneider. Weak gravitational lensing. Physics Reports, 340(4-5): 291-472, January 2001. doi: 10.1016/s0370-1573(00)00082-x.

D. Baumann, H. S. Chia, and R. A. Porto. Probing Ultralight Bosons with Binary Black Holes. arXiv, April 2018. 1804.03208.

J. Baur, N. Palanque-Delabrouille, C. Yèche, C. Magneville, and M. Viel. Lyman-alpha forests cool warm dark matter. Journal of Cosmology and Astroparticle Physics, 2016 (08):012-012, August 2016. doi: 10.1088/1475-7516/2016/08/012.

G. D. Becker, J. S. Bolton, M. G. Haehnelt, and W. L. W. Sargent. Detection of extended He ii reionization in the temperature evolution of the intergalactic medium. Monthly Notices of the Royal Astronomical Society, 410(2):1096-1112, November 2010. doi: 10. 1111/j.1365-2966.2010.17507.x.

R. H. Becker, X. Fan, R. L. White, M. A. Strauss, V. K. Narayanan, R. H. Lupton, J. E. Gunn, J. Annis, N. A. Bahcall, J. Brinkmann, A. J. Connolly, I. Csabai, P. C. Czarapata, M. Doi, T. M. Heckman, G. S. Hennessy, Ž. Ivezić, G. R. Knapp, D. Q. Lamb, T. A. McKay, J. A. Munn, T. Nash, R. Nichol, J. R. Pier, G. T. Richards, D. P. Schneider, 
C. Stoughton, A. S. Szalay, A. R. Thakar, and D. G. York. Evidence for reionization at $\mathrm{z} \sim 6$ : Detection of a gunn-peterson trough in a $\mathrm{z}=6.28$ quasar. The Astronomical Journal, 122(6):2850-2857, December 2001. doi: 10.1086/324231.

J. D. Bekenstein. Extraction of energy and charge from a black hole. Physical Review D, 7(4):949-953, February 1973. doi: 10.1103/physrevd.7.949.

A. J. Benson. Galacticus: A semi-analytic model of galaxy formation. New Astronomy, 17 (2):175-197, February 2012. doi: 10.1016/j.newast.2011.07.004.

A. J. Benson, A. Farahi, S. Cole, L. A. Moustakas, A. Jenkins, M. Lovell, R. Kennedy, J. Helly, and C. Frenk. Dark matter halo merger histories beyond cold dark matter i. methods and application to warm dark matter. Monthly Notices of the Royal Astronomical Society, 428(2):1774-1789, November 2012. doi: 10.1093/mnras/sts159.

A. Bernal and F. S. Guzmán. Scalar field dark matter: Head-on interaction between two structures. Phys. Rev. D, 74:103002, November 2006. doi: 10.1103/PhysRevD.74.103002.

A. Bernal and F. S. Guzmán. Scalar field dark matter: Nonspherical collapse and late-time behavior. Physical Review D, 74(6), September 2006. doi: 10.1103/physrevd.74.063504.

T. Bernal, L. M. Fernández-Hernández, T. Matos, and M. A. Rodríguez-Meza. Rotation curves of high-resolution LSB and SPARC galaxies with fuzzy and multistate (ultralight boson) scalar field dark matter. Monthly Notices of the Royal Astronomical Society, 475 (2):1447-1468, December 2017. doi: 10.1093/mnras/stx3208.

M. Bezares, C. Palenzuela, and C. Bona. Final fate of compact boson star mergers. Physical Review D, 95(12), June 2017. doi: 10.1103/physrevd.95.124005.

S. Bharadwaj and S. S. Ali. The cosmic microwave background radiation fluctuations from $\mathrm{h}$ i perturbations prior to reionization. Monthly Notices of the Royal Astronomical Society, 352(1):142-146, July 2004. doi: 10.1111/j.1365-2966.2004.07907.x.

S. Bharadwaj and S. S. Ali. On using visibility correlations to probe the H i distribution from the dark ages to the present epoch - I. Formalism and the expected signal. Monthly Notices of the Royal Astronomical Society, 356(4):1519-1528, February 2005. doi: 10. 1111/j.1365-2966.2004.08604.x.

P. Bode, J. P. Ostriker, and N. Turok. Halo formation in warm dark matter models. The Astrophysical Journal, 556(1):93-107, July 2001. doi: 10.1086/321541.

D. Bohm. A suggested interpretation of the quantum theory in terms of "hidden" variables. i. Phys. Rev., 85:166-179, January 1952. doi: 10.1103/PhysRev.85.166.

J. S. Bolton, E. Puchwein, D. Sijacki, M. G. Haehnelt, T.-S. Kim, A. Meiksin, J. A. Regan, and M. Viel. The Sherwood simulation suite: overview and data comparisons with the Lyman $\alpha$ forest at redshifts $2 \leq \mathrm{z} \leq 5$. Monthly Notices of the Royal Astronomical Society, 464(1):897-914, September 2016. doi: 10.1093/mnras/stw2397. 
J. R. Bond, S. Cole, G. Efstathiou, and N. Kaiser. Excursion set mass functions for hierarchical gaussian fluctuations. The Astrophysical Journal, 379:440, October 1991. doi: $10.1086 / 170520$.

S. Bose, A. J. Deason, and C. S. Frenk. The imprint of cosmic reionisation on the luminosity function of galaxies. arXiv, February 2018. 1802.10096.

M. Bošković, F. Duque, M. C. Ferreira, F. S. Miguel, and V. Cardoso. Motion in timeperiodic backgrounds with applications to ultralight dark matter halos at galactic centers. Physical Review D, 98(2), July 2018. doi: 10.1103/physrevd.98.024037.

R. J. Bouwens, G. D. Illingworth, P. A. Oesch, J. Caruana, B. Holwerda, R. Smit, and S. Wilkins. Reionization after planck: The derived growth of the cosmic ionizing emissivity now matches the growth of the galaxy uv luminosity density. The Astrophysical Journal, 811(2):140, September 2015a. doi: 10.1088/0004-637x/811/2/140.

R. J. Bouwens, G. D. Illingworth, P. A. Oesch, M. Trenti, I. Labbé, L. Bradley, M. Carollo, P. G. van Dokkum, V. Gonzalez, B. Holwerda, M. Franx, L. Spitler, R. Smit, and D. Magee. UV luminosity functions at redshifts $\mathrm{z} \sim 4$ to $\mathrm{z} \sim 10$ : 10000 galaxies from hst legacy fields. The Astrophysical Journal, 803(1):34, April 2015b. doi: 10.1088/ 0004-637x/803/1/34.

J. D. Bowman, A. E. E. Rogers, R. A. Monsalve, T. J. Mozdzen, and N. Mahesh. An absorption profile centred at 78 megahertz in the sky-averaged spectrum. Nature, 555 (7694):67-70, February 2018. doi: 10.1038/nature25792.

M. Boylan-Kolchin, V. Springel, S. D. M. White, A. Jenkins, and G. Lemson. Resolving cosmic structure formation with the millennium-II simulation. Monthly Notices of the Royal Astronomical Society, 398(3):1150-1164, September 2009. doi: 10.1111/j.1365-2966.2009.15191.x.

M. Boylan-Kolchin, J. S. Bullock, and M. Kaplinghat. Too big to fail? The puzzling darkness of massive Milky Way subhaloes. Monthly Notices of the Royal Astronomical Society: Letters, 415(1):L40-L44, June 2011. doi: 10.1111/j.1745-3933.2011.01074.x.

B. Bozek, D. J. E. Marsh, J. Silk, and R. F. G. Wyse. Galaxy UV-luminosity function and reionization constraints on axion dark matter. Monthly Notices of the Royal Astronomical Society, 450(1):209-222, April 2015. doi: 10.1093/mnras/stv624.

E. Braaten, A. Mohapatra, and H. Zhang. Dense axion stars. Physical Review Letters, 117 (12), September 2016. doi: 10.1103/physrevlett.117.121801.

M. A. Breddels and A. Helmi. Model comparison of the dark matter profiles of Fornax, Sculptor, Carina and Sextans. Astronomy 83 Astrophysics, 558:A35, October 2013. doi: 10.1051/0004-6361/201321606. 
D. R. Brill, P. L. Chrzanowski, C. M. Pereira, E. D. Fackerell, and J. R. Ipser. Solution of the scalar wave equation in a kerr background by separation of variables. Physical Review D, 5(8):1913-1915, April 1972. doi: 10.1103/physrevd.5.1913.

R. Brito, V. Cardoso, and P. Pani. Superradiance. Springer International Publishing, 2015. doi: 10.1007/978-3-319-19000-6.

R. Brito, S. Ghosh, E. Barausse, E. Berti, V. Cardoso, I. Dvorkin, A. Klein, and P. Pani. Gravitational wave searches for ultralight bosons with LIGO and LISA. Physical Review D, 96(6), September 2017a. doi: 10.1103/physrevd.96.064050.

R. Brito, S. Ghosh, E. Barausse, E. Berti, V. Cardoso, I. Dvorkin, A. Klein, and P. Pani. Stochastic and resolvable gravitational waves from ultralight bosons. Physical Review Letters, 119(13), September 2017b. doi: 10.1103/physrevlett.119.131101.

T. J. Broadhurst, A. N. Taylor, and J. A. Peacock. Mapping cluster mass distributions via gravitational lensing of background galaxies. The Astrophysical Journal, 438:49, January 1995. doi: $10.1086 / 175053$.

G. L. Bryan and M. L. Norman. Statistical properties of x-ray clusters: Analytic and numerical comparisons. The Astrophysical Journal, 495(1):80-99, March 1998. doi: $10.1086 / 305262$.

G. L. Bryan, M. L. Norman, B. W. O'Shea, T. Abel, J. H. Wise, M. J. Turk, D. R. Reynolds, D. C. Collins, P. Wang, S. W. Skillman, B. Smith, R. P. Harkness, J. Bordner, J.-h. Kim, M. Kuhlen, H. Xu, N. Goldbaum, C. Hummels, A. G. Kritsuk, E. Tasker, S. Skory, C. M. Simpson, O. Hahn, J. S. Oishi, G. C. So, F. Zhao, R. Cen, Y. Li, and The Enzo Collaboration. ENZO: An Adaptive Mesh Refinement Code for Astrophysics. apjs, 211:19, April 2014. doi: 10.1088/0067-0049/211/2/19.

M. R. Buckley and A. H. G. Peter. Gravitational probes of dark matter physics. arXiv, December 2017. 1712.06615.

J. S. Bullock. Notes on the Missing Satellites Problem. arXiv, September 2010. 1009.4505.

J. S. Bullock and M. Boylan-Kolchin. Small-Scale Challenges to the $\Lambda$ CDM Paradigm. Annual Review of Astronomy and Astrophysics, 55:343-387, August 2017. doi: 10.1146/ annurev-astro-091916-055313.

J. S. Bullock, A. V. Kravtsov, and D. H. Weinberg. Reionization and the abundance of galactic satellites. The Astrophysical Journal, 539(2):517-521, August 2000. doi: $10.1086 / 309279$.

J. S. Bullock, A. Dekel, T. S. Kolatt, A. V. Kravtsov, A. A. Klypin, C. Porciani, and J. R. Primack. A universal angular momentum profile for galactic halos. The Astrophysical Journal, 555(1):240-257, July 2001. doi: 10.1086/321477. 
A. J. Bunker, E. R. Stanway, R. S. Ellis, and R. G. McMahon. The star formation rate of the universe at $\mathrm{z} \approx 6$ from the hubble ultra-deep field. Monthly Notices of the Royal Astronomical Society, 355(2):374-384, December 2004. doi: 10.1111/j.1365-2966.2004. 08326.x.

A. Burkert. The structure of dark matter halos in dwarf galaxies. The Astrophysical Journal, 447(1), July 1995. doi: 10.1086/309560.

A. Burkert. The Structure and Dark Halo Core Properties of Dwarf Spheroidal Galaxies. arXiv, January 2015. 1501.06604.

E. Calabrese and D. N. Spergel. Ultra-light dark matter in ultra-faint dwarf galaxies. Monthly Notices of the Royal Astronomical Society, 460(4):4397-4402, May 2016. doi: $10.1093 / \mathrm{mnras} / \mathrm{stw} 1256$.

V. Cardoso, Ó. J. C. Dias, G. S. Hartnett, M. Middleton, P. Pani, and J. E. Santos. Constraining the mass of dark photons and axion-like particles through black-hole superradiance. Journal of Cosmology and Astroparticle Physics, 2018(03):043-043, March 2018. doi: 10.1088/1475-7516/2018/03/043.

N. D. Cartwright. A non-negative Wigner-type distribution. Physica A: Statistical Mechanics and its Applications, 83(1):210-212, 1976. doi: 10.1016/0378-4371(76)90145-x.

F. X. L. Cedeño, A. X. González-Morales, and L. A. Ureña-López. Cosmological signatures of ultralight dark matter with an axionlike potential. Physical Review D, 96(6), September 2017. doi: 10.1103/physrevd.96.061301.

S. S. Chakrabarty and P. Sikivie. Effects of a caustic ring of dark matter on the distribution of stars and interstellar gas. arXiv, July 2018. 1808.00027.

J. H. H. Chan, H.-Y. Schive, T.-P. Woo, and T. Chiueh. How do stars affect $\psi$ DM haloes? Monthly Notices of the Royal Astronomical Society, 478(2):2686-2699, April 2018. doi: $10.1093 / \mathrm{mnras} / \mathrm{sty} 900$.

S.-R. Chen, H.-Y. Schive, and T. Chiueh. Jeans analysis for dwarf spheroidal galaxies in wave dark matter. Monthly Notices of the Royal Astronomical Society, 468(2):1338-1348, February 2017. doi: 10.1093/mnras/stx449.

X. Chen and J. Miralda-Escude. The Spin-Kinetic Temperature Coupling and the Heating Rate due to Ly $\alpha$ Scattering before Reionization: Predictions for 21 Centimeter Emission and Absorption. The Astrophysical Journal, 602(1):1-11, February 2004. doi: 10.1086/ 380829.

K. Choi, C. Rott, and Y. Itow. Impact of the dark matter velocity distribution on capture rates in the Sun. Journal of Cosmology and Astroparticle Physics, 2014(05):049-049, May 2014. doi: 10.1088/1475-7516/2014/05/049. 
T. R. Choudhury, M. G. Haehnelt, and J. Regan. Inside-out or outside-in: the topology of reionization in the photon-starved regime suggested by Ly $\alpha$ forest data. Monthly Notices of the Royal Astronomical Society, 394(2):960-977, April 2009. doi: 10.1111/j. 1365-2966.2008.14383.x.

D. Christodoulou. Reversible and Irreversible Transformations in Black-Hole Physics. Physical Review Letters, 25(22):1596-1597, November 1970. doi: 10.1103/physrevlett.25. 1596.

K. Clough, T. Dietrich, and J. C. Niemeyer. Axion star collisions with black holes and neutron stars in full 3D numerical relativity. arXiv, August 2018. 1808.04668.

S. Cole, C. G. Lacey, C. M. Baugh, and C. S. Frenk. Hierarchical galaxy formation. Mon.Not.Roy.Astron.Soc., 319:168-204, November 2000. doi: 10.1046/j.1365-8711.2000. 03879.x.

S. Coleman. Aspects of Symmetry. Cambridge University Press, 1985. doi: 10.1017/ cbo9780511565045.

P. Coles and K. Spencer. A wave-mechanical approach to cosmic structure formation. Monthly Notices of the Royal Astronomical Society, 342(1):176-184, June 2003. doi: 10.1046/j.1365-8711.2003.06529.x.

A. Cooray and M. Milosavljević. What is L $\star$ ? Anatomy of the Galaxy Luminosity Function. The Astrophysical Journal, 627(2):L89-L92, June 2005. doi: 10.1086/432259.

P.S. Corasaniti, S. Agarwal, D. J.E. Marsh, and S. Das. Constraints on dark matter scenarios from measurements of the galaxy luminosity function at high redshifts. Physical Review D, 95(8), April 2017. doi: 10.1103/physrevd.95.083512.

E. Cotner. Collisional interactions between self-interacting nonrelativistic boson stars: Effective potential analysis and numerical simulations. Physical Review D, 94(6), September 2016. doi: 10.1103/physrevd.94.063503.

N. H. M. Crighton, M. T. Murphy, J. X. Prochaska, G. Worseck, M. Rafelski, G. D. Becker, S. L. Ellison, M. Fumagalli, S. Lopez, A. Meiksin, and J. M. O'Meara. The neutral hydrogen cosmological mass density at $\mathrm{z}=5$. Monthly Notices of the Royal Astronomical Society, 452(1):217-234, July 2015. doi: 10.1093/mnras/stv1182.

H. Deng, M. P. Hertzberg, M. H. Namjoo, and A. Masoumi. Can light dark matter solve the core-cusp problem? Physical Review D, 98(2), July 2018. doi: 10.1103/physrevd.98. 023513.

S. Detweiler. Klein-Gordon equation and rotating black holes. Physical Review D, 22(10): 2323-2326, November 1980. doi: 10.1103/physrevd.22.2323. 
J. M. Diego, T. Broadhurst, C. Chen, J. Lim, A. Zitrin, B. Chan, D. Coe, H. C. Ford, D. Lam, and W. Zheng. A free-form prediction for the reappearance of supernova Refsdal in the Hubble Frontier Fields cluster MACSJ1149.5+2223. Monthly Notices of the Royal Astronomical Society, 456(1):356-365, December 2015. doi: 10.1093/mnras/stv2638.

J. Diemand, M. Kuhlen, P. Madau, M. Zemp, B. Moore, D. Potter, and J. Stadel. Clumps and streams in the local dark matter distribution. Nature, 454(7205):735-738, August 2008. doi: 10.1038/nature07153.

T. Dietrich, F. Day, K. Clough, M. Coughlin, and J. Niemeyer. Neutron star - axion star collisions in the light of multi-messenger astronomy. arXiv, August 2018. 1808.04746.

M. Dijkstra. Ly $\alpha$ Emitting Galaxies as a Probe of Reionisation. Publications of the Astronomical Society of Australia, 31, 2014. doi: 10.1017/pasa.2014.33.

M. Dijkstra, M. Gronke, and A. Venkatesan. The LyA-LyC Connection: Evidence for an Enhanced Contribution of UV-faint Galaxies to Cosmic Reionization. The Astrophysical Journal, 828(2):71, September 2016. doi: 10.3847/0004-637x/828/2/71.

S. G. Djorgovski, S. Castro, D. Stern, and A. A. Mahabal. On the Threshold of the Reionization Epoch. The Astrophysical Journal, 560(1):L5-L8, October 2001. doi: 10. $1086 / 324175$.

S. R. Dolan. Instability of the massive Klein-Gordon field on the Kerr spacetime. Physical Review D, 76(8), October 2007. doi: 10.1103/physrevd.76.084001.

F. Donato, G. Gentile, P. Salucci, C. Frigerio Martins, M. I. Wilkinson, G. Gilmore, E. K. Grebel, A. Koch, and R. Wyse. A constant dark matter halo surface density in galaxies. Monthly Notices of the Royal Astronomical Society, 397(3):1169-1176, August 2009. doi: 10.1111/j.1365-2966.2009.15004.x.

X. Du, C. Behrens, and J. C. Niemeyer. Substructure of fuzzy dark matter haloes. Monthly Notices of the Royal Astronomical Society, 465(1):941-951, October 2016. doi: 10.1093/ mnras/stw2724.

X. Du, C. Behrens, J. C. Niemeyer, and B. Schwabe. Core-halo mass relation of ultralight axion dark matter from merger history. Physical Review D, 95(4), February 2017. doi: 10.1103/physrevd.95.043519.

X. Du, B. Schwabe, J. C. Niemeyer, and D. Bürger. Tidal disruption of fuzzy dark matter subhalo cores. Physical Review D, 97(6), March 2018. doi: 10.1103/physrevd.97.063507.

A. A. Dutton, A. V. Macciò, J. Frings, L. Wang, G. S. Stinson, C. Penzo, and X. Kang. NIHAO V: too big does not fail reconciling the conflict between $\Lambda$ CDM predictions and the circular velocities of nearby field galaxies. Monthly Notices of the Royal Astronomical Society: Letters, 457(1):L74-L78, March 2016. doi: 10.1093/mnrasl/slv193. 
J. Eby, M. Leembruggen, J. Leeney, P. Suranyi, and L. C. R. Wijewardhana. Collisions of dark matter axion stars with astrophysical sources. Journal of High Energy Physics, 2017(4), April 2017. doi: 10.1007/jhep04(2017)099.

F. Edwards, E. Kendall, S. Hotchkiss, and R. Easther. PyUltraLight: A Pseudo-Spectral Solver for Ultralight Dark Matter Dynamics. arXiv, July 2018. 1807.04037.

G. Efstathiou. Suppressing the formation of dwarf galaxies via photoionization. Monthly Notices of the Royal Astronomical Society, 256(1):43P-47P, May 1992. doi: 10.1093/ mnras/256.1.43p.

O. D. Elbert, J. S. Bullock, S. Garrison-Kimmel, M. Rocha, J. Oñorbe, and A. H. G. Peter. Core formation in dwarf haloes with self-interacting dark matter: no fine-tuning necessary. Monthly Notices of the Royal Astronomical Society, 453(1):29-37, August 2015. doi: $10.1093 / \mathrm{mnras} / \mathrm{stv} 1470$.

O. D. Elbert, J. S. Bullock, M. Kaplinghat, S. Garrison-Kimmel, A. S. Graus, and M. Rocha. A Testable Conspiracy: Simulating Baryonic Effects on Self-interacting Dark Matter Halos. The Astrophysical Journal, 853(2):109, January 2018. doi: 10.3847/1538-4357/aa9710.

R. Emami, T. Broadhurst, G. Smoot, T. Chiueh, and L. H. Nhan. A Soliton Solution for the Central Dark Masses in Globular Clusters and Implications for the Axiverse. arXiv, June 2018. 1806.04518.

S. Endlich and R. Penco. A modern approach to superradiance. Journal of High Energy Physics, 2017(5), May 2017. doi: 10.1007/jhep05(2017)052.

B. Engquist and O. Runborg. Computational high frequency wave propagation. Acta Numerica, 12:181-266, May 2003. doi: 10.1017/s0962492902000119.

Euclid Collaboration. Cosmology and Fundamental Physics with the Euclid Satellite. Living Reviews in Relativity, 16(1), September 2013. doi: 10.12942/lrr-2013-6.

X. Fan, M. A. Strauss, R. H. Becker, R. L. White, J. E. Gunn, G. R. Knapp, G. T. Richards, D. P. Schneider, J. Brinkmann, and M. Fukugita. Constraining the Evolution of the Ionizing Background and the Epoch of Reionization with z 6 Quasars. II. A Sample of 19 Quasars. The Astronomical Journal, 132(1):117-136, June 2006. doi: $10.1086 / 504836$.

F. Feroz, M.P. Hobson, and M. Bridges. MultiNest: an efficient and robust Bayesian inference tool for cosmology and particle physics. Monthly Notices of the Royal Astronomical Society, 398(4):1601-1614, October 2009. doi: 10.1111/j.1365-2966.2009.14548.x.

G. Field. Excitation of the Hydrogen 21-CM Line. Proceedings of the IRE, 46(1):240-250, January 1958. doi: 10.1109/jrproc.1958.286741. 
G. B. Field. The Spin Temperature of Intergalactic Neutral Hydrogen. The Astrophysical Journal, 129:536, May 1959. doi: 10.1086/146653.

K. Finlator, M. K. M. Prescott, B. D. Oppenheimer, R. Davé, E. Zackrisson, R. C. Livermore, S. L. Finkelstein, R. Thompson, and S. Huang. The minimum halo mass for star formation at $\mathrm{z}=6-8$. Monthly Notices of the Royal Astronomical Society, 464(2): 1633-1639, September 2016. doi: 10.1093/mnras/stw2433.

A. Fitts, M. Boylan-Kolchin, O. D. Elbert, J. S. Bullock, P. F. Hopkins, J. Oñorbe, A. Wetzel, C. Wheeler, C. Faucher-Giguère, D. Kereš, E. D. Skillman, and D. R. Weisz. Fire in the field: simulating the threshold of galaxy formation. Monthly Notices of the Royal Astronomical Society, 471(3):3547-3562, July 2017. doi: 10.1093/mnras/stx1757.

R. A. Flores and J. R. Primack. Observational and theoretical constraints on singular dark matter halos. The Astrophysical Journal, 427:L1, May 1994. doi: 10.1086/187350.

A. Font-Ribera, J. Miralda-Escudé, E. Arnau, B. Carithers, K. Lee, P. Noterdaeme, I. Pâris, P.Petitjean, J. Rich, E. Rollinde, N. P. Ross, D. P. Schneider, M. White, and D. G. York. The large-scale cross-correlation of Damped Lyman alpha systems with the Lyman alpha forest: first measurements from BOSS. Journal of Cosmology and Astroparticle Physics, 2012(11):059-059, November 2012. doi: 10.1088/1475-7516/2012/11/059.

F. Fontanot, S. Cristiani, and E. Vanzella. On the relative contribution of high-redshift galaxies and active galactic nuclei to reionization. Monthly Notices of the Royal Astronomical Society, 425(2):1413-1420, August 2012. doi: 10.1111/j.1365-2966.2012.21594.x.

A. B. Fry, F. Governato, A. Pontzen, T. Quinn, M. Tremmel, L. Anderson, H. Menon, A. M. Brooks, and J. Wadsley. All about baryons: revisiting SIDM predictions at small halo masses. Monthly Notices of the Royal Astronomical Society, 452(2):1468-1479, July 2015. doi: 10.1093/mnras/stv1330.

S. R. Furlanetto. The global 21-centimeter background from high redshifts. Monthly Notices of the Royal Astronomical Society, 371(2):867-878, September 2006. doi: 10. 1111/j.1365-2966.2006.10725.x.

S. R. Furlanetto, M. Zaldarriaga, and L. Hernquist. The Growth of HiiRegions During Reionization. The Astrophysical Journal, 613(1):1-15, September 2004. doi: 10.1086/ 423025.

S. R. Furlanetto, S. P. Oh, and F. H. Briggs. Cosmology at low frequencies: The 21cm transition and the high-redshift Universe. Physics Reports, 433(4-6):181-301, October 2006. doi: 10.1016/j.physrep.2006.08.002.

Gaia Collaboration. Gaia Data Release 2. Astronomy \& Astrophysics, 616:A1, August 2018. doi: 10.1051/0004-6361/201833051. 
A. B. Gaina. On the possibility of the formation of a condensate by primordial black holes. Sov. Astron. Lett., 15:243-245, 1989.

J. P. Gardner, J. C. Mather, M. Clampin, R. Doyon, M. A. Greenhouse, H. B. Hammel, J. B. Hutchings, P. Jakobsen, S. J. Lilly, K. S. Long, J. I. Lunine, M. J. Mccaughrean, M. Mountain, J. Nella, G. H. Rieke, M. J. Rieke, H.-W. Rix, E. P. Smith, G. Sonneborn, M. Stiavelli, H. S. Stockman, R. A. Windhorst, and G. S. Wright. The James Webb Space Telescope. Space Science Reviews, 123(4):485-606, November 2006. doi: 10.1007/ s11214-006-8315-7.

M. Garny and T. Konstandin. Gravitational collapse in the Schrdinger-Poisson system. Journal of Cosmology and Astroparticle Physics, 2018(01):009-009, January 2018. doi: 10.1088/1475-7516/2018/01/009.

S. Garrison-Kimmel, M. Boylan-Kolchin, J. S. Bullock, and K. Lee. ELVIS: Exploring the Local Volume in Simulations. Monthly Notices of the Royal Astronomical Society, 438 (3):2578-2596, January 2014. doi: 10.1093/mnras/stt2377.

S. Garrison-Kimmel, A. Wetzel, J. S. Bullock, P. F. Hopkins, M. Boylan-Kolchin, C.-A. Faucher-Giguère, D. Kereš, E. Quataert, R. E. Sanderson, A. S. Graus, and T. Kelley. Not so lumpy after all: modelling the depletion of dark matter subhaloes by Milky Waylike galaxies. Monthly Notices of the Royal Astronomical Society, 471(2):1709-1727, July 2017. doi: $10.1093 / \mathrm{mnras} / \mathrm{stx} 1710$.

E. M. George, C. L. Reichardt, K. A. Aird, B. A. Benson, L. E. Bleem, J. E. Carlstrom, C. L. Chang, H-M. Cho, T. M. Crawford, A. T. Crites, T. de Haan, M. A. Dobbs, J. Dudley, N. W. Halverson, N. L. Harrington, G. P. Holder, W. L. Holzapfel, Z. Hou, J. D. Hrubes, R. Keisler, L. Knox, A. T. Lee, E. M. Leitch, M. Lueker, D. Luong-Van, J. J. McMahon, J. Mehl, S. S. Meyer, M. Millea, L. M. Mocanu, J. J. Mohr, T. E. Montroy, S. Padin, T. Plagge, C. Pryke, J. E. Ruhl, K. K. Schaffer, L. Shaw, E. Shirokoff, H. G. Spieler, Z. Staniszewski, A. A. Stark, K. T. Story, A. van Engelen, K. Vanderlinde, J. D. Vieira, R. Williamson, and O. Zahn. A Measurement of Secondary Cosmic Microwave Background Anisotropies from the 2500 Square-degree SPT-SZ Survey. The Astrophysical Journal, 799(2):177, January 2015. doi: 10.1088/0004-637x/799/2/177.

O. E. Gerhard and D. N. Spergel. Dwarf spheroidal galaxies and the mass of the neutrino. The Astrophysical Journal, 389:L9, April 1992. doi: 10.1086/186336.

A. Goldberg, H. M. Schey, and J. L. Schwartz. Computer-Generated Motion Pictures of One-Dimensional Quantum-Mechanical Transmission and Reflection Phenomena. American Journal of Physics, 35(3):177-186, March 1967. doi: 10.1119/1.1973991.

J. A González and F. S. Guzmán. Interference pattern in the collision of structures in the Bose-Einstein condensate dark matter model: Comparison with fluids. Physical Review D, 83(10), May 2011. doi: 10.1103/physrevd.83.103513. 
A. X. González-Morales, D. J. E. Marsh, J. Peñarrubia, and L. A. Ureña-López. Unbiased constraints on ultralight axion mass from dwarf spheroidal galaxies. Monthly Notices of the Royal Astronomical Society, 472(2):1346-1360, August 2017. doi: 10.1093/mnras/ stx1941.

F. Governato, A. Zolotov, A. Pontzen, C. Christensen, S. H. Oh, A. M. Brooks, T. Quinn, S. Shen, and J. Wadsley. Cuspy no more: how outflows affect the central dark matter and baryon distribution in $\Lambda$ cold dark matter galaxies. mnras, 422:1231-1240, May 2012. doi: $10.1111 /$ j.1365-2966.2012.20696.x.

J. E. Gunn and B. A. Peterson. On the Density of Neutral Hydrogen in Intergalactic Space. The Astrophysical Journal, 142:1633, November 1965. doi: 10.1086/148444.

F. S. Guzmán and L. A. Ureña-López. Evolution of the Schrdinger-Newton system for a self-gravitating scalar field. Physical Review D, 69(12), June 2004. doi: 10.1103/ physrevd.69.124033.

F. S. Guzmán and A. A. Avilez. Head-on collision of multistate ultralight BEC dark matter configurations. Physical Review D, 97(11), June 2018. doi: 10.1103/physrevd.97.116003.

F. S. Guzmán, J. A. González, and J.P. Cruz-Pérez. Behavior of luminous matter in the head-on encounter of two ultralight BEC dark matter halos. Physical Review D, 93(10), May 2016. doi: 10.1103/physrevd.93.103535.

O. Hahn and T. Abel. Multi-scale initial conditions for cosmological simulations. Monthly Notices of the Royal Astronomical Society, 415(3):2101-2121, July 2011. doi: 10.1111/ j.1365-2966.2011.18820.x.

Z. Haiman and A. Loeb. Observational Signatures of the First Quasars. The Astrophysical Journal, 503(2):505-517, August 1998. doi: 10.1086/306017.

C. Harabati, J. M. Rost, and F. Grossmann. Long-time and unitary properties of semiclassical initial value representations. The Journal of Chemical Physics, 120(1):26-30, January 2004. doi: 10.1063/1.1630033.

Y. He. Calabi-Yau Geometries: Algorithms, Databases, and Physics. International Journal of Modern Physics A, 28(21):1330032, August 2013. doi: 10.1142/s0217751x13300329.

T. Helfer, D. J.E. Marsh, K. Clough, M. Fairbairn, E. A. Lim, and R. Becerril. Black hole formation from axion stars. Journal of Cosmology and Astroparticle Physics, 2017(03): 055-055, March 2017. doi: 10.1088/1475-7516/2017/03/055.

T. Helfer, E. A. Lim, M. A. G. Garcia, and M. A. Amin. Gravitational Wave Emission from Collisions of Compact Scalar Solitons. arXiv, February 2018. 1802.06733. 
M. F. Herman and E. Kluk. A semiclasical justification for the use of non-spreading wavepackets in dynamics calculations. Chemical Physics, 91(1):27-34, November 1984. doi: 10.1016/0301-0104(84)80039-7.

A. Herrera-Martín, M. Hendry, A. X. Gonzalez-Morales, and L. A. Ureña-López. Gravitational lensing by wave dark matter halos. arXiv, July 2017. 1707.09929.

G. J. Hill, K. Gebhardt, E. Komatsu, N. Drory, P. J. MacQueen, J. Adams, G. A. Blanc, R. Koehler, M. Rafal, M. M. Roth, A. Kelz, C. Gronwall, R. Ciardullo, and D. P. Schneider. The Hobby-Eberly Telescope Dark Energy Experiment (HETDEX): Description and Early Pilot Survey Results. arXiv, June 2008. 0806.0183.

R. Hills, G. Kulkarni, P. D. Meerburg, and E. Puchwein. Concerns about Modelling of Foregrounds and the 21-cm Signal in EDGES data. arXiv, May 2018. 1805.01421.

C. M. Hirata. Wouthuysen-Field coupling strength and application to high-redshift 21-cm radiation. Monthly Notices of the Royal Astronomical Society, 367(1):259-274, March 2006. doi: $10.1111 /$ j.1365-2966.2005.09949.x.

R. Hložek, D. Grin, D. J. E. Marsh, and P. G. Ferreira. A search for ultralight axions using precision cosmological data. Physical Review D, 91(10), May 2015. doi: 10.1103/ physrevd.91.103512.

R. Hložek, D. J. E. Marsh, D. Grin, R. Allison, J. Dunkley, and E. Calabrese. Future CMB tests of dark matter: Ultralight axions and massive neutrinos. Physical Review D, 95 (12), June 2017. doi: 10.1103/physrevd.95.123511.

R. Hložek, D.J.E. Marsh, and D. Grin. Using the full power of the cosmic microwave background to probe axion dark matter. Monthly Notices of the Royal Astronomical Society, 476(3):3063-3085, February 2018. doi: 10.1093/mnras/sty271.

P. F. Hopkins, A. Wetzel, D. Kereš, C.-A. Faucher-Giguère, E. Quataert, M. BoylanKolchin, N. Murray, C. C. Hayward, S. Garrison-Kimmel, C. Hummels, R. Feldmann, P. Torrey, X. Ma, D. Anglés-Alcázar, K.-Y. Su, M. Orr, D. Schmitz, I. Escala, R. Sanderson, M. Y Grudić, Z. Hafen, J.-H. Kim, A. Fitts, J. S. Bullock, C. Wheeler, T. K. Chan, O. D. Elbert, and D. Narayanan. FIRE-2 simulations: physics versus numerics in galaxy formation. Monthly Notices of the Royal Astronomical Society, 480(1):800-863, June 2018. doi: 10.1093/mnras/sty1690.

J. R. Hörandel, L. Bähren, S. Buitink, H. Falcke, A. Horneffer, J. Kuijpers, S. Lafèbre, A. Nigl, O. Scholten, and K. Singh. LOFAR - A new experiment to record radio emission from cosmic particles. Nuclear Physics B - Proceedings Supplements, 196:289-292, December 2009. doi: 10.1016/j.nuclphysbps.2009.09.055.

W. Hu, R. Barkana, and A. Gruzinov. Fuzzy Cold Dark Matter: The Wave Properties of Ultralight Particles. Physical Review Letters, 85(6):1158-1161, August 2000. doi: 10.1103/physrevlett.85.1158. 
L. Hui and Z. Haiman. The Thermal Memory of Reionization History. The Astrophysical Journal, 596(1):9-18, October 2003. doi: 10.1086/377229.

L. Hui, J. P. Ostriker, S. Tremaine, and E. Witten. Ultralight scalars as cosmological dark matter. Physical Review D, 95(4), February 2017. doi: 10.1103/physrevd.95.043541.

J.-C. Hwang and H. Noh. Axion as a Cold Dark Matter Candidate. Physics Letters B, 680 (1):1-3, September 2009. doi: 10.1016/j.physletb.2009.08.031.

V. Iršič, M. Viel, M. G. Haehnelt, J. S. Bolton, and G. D. Becker. First Constraints on Fuzzy Dark Matter from Lyman- $\alpha$ Forest Data and Hydrodynamical Simulations. Physical Review Letters, 119(3), July 2017. doi: 10.1103/physrevlett.119.031302.

Y. I. Izotov and T. X. Thuan. Systematic Effects and a New Determination of the Primordial Abundance of $4 \mathrm{He}$ and $\mathrm{dY} / \mathrm{dZ}$ from Observations of Blue Compact Galaxies. The Astrophysical Journal, 602(1):200-230, February 2004. doi: 10.1086/380830.

S. U. Ji and S. J. Sin. Late-time phase transition and the galactic halo as a Bose liquid. II. The effect of visible matter. Physical Review D, 50(6):3655-3659, September 1994. doi: $10.1103 /$ physrevd.50.3655.

M. Kaplinghat, S. Tulin, and H.-B. Yu. Dark Matter Halos as Particle Colliders: Unified Solution to Small-Scale Structure Puzzles from Dwarfs to Clusters. Physical Review Letters, 116(4), January 2016. doi: 10.1103/physrevlett.116.041302.

K. G. Kay. Integral expressions for the semiclassical time-dependent propagator. The Journal of Chemical Physics, 100(6):4377-4392, March 1994. doi: 10.1063/1.466320.

K. G. Kay. The Herman-Kluk approximation: Derivation and semiclassical corrections. Chemical Physics, 322(1-2):3-12, March 2006. doi: 10.1016/j.chemphys.2005.06.019.

M. Y. Khlopov, B. A. Malomed, and Y. B. Zeldovich. Gravitational instability of scalar fields and formation of primordial black holes. Monthly Notices of the Royal Astronomical Society, 215(4):575-589, August 1985. doi: 10.1093/mnras/215.4.575.

A. Khmelnitsky and V. Rubakov. Pulsar timing signal from ultralight scalar dark matter. Journal of Cosmology and Astroparticle Physics, 2014(02):019-019, February 2014. doi: 10.1088/1475-7516/2014/02/019.

M. Kilbinger. Cosmological parameters from weak cosmological lensing. arXiv, July 2018. 1807.08249 .

E. Kluk, M. F. Herman, and H. L. Davis. Comparison of the propagation of semiclassical frozen Gaussian wave functions with quantum propagation for a highly excited anharmonic oscillator. The Journal of Chemical Physics, 84(1):326-334, January 1986. doi: $10.1063 / 1.450142$. 
A. Klypin, A. V. Kravtsov, O. Valenzuela, and F. Prada. Where Are the Missing Galactic Satellites? The Astrophysical Journal, 522(1):82-92, September 1999. doi: 10.1086/ 307643.

T. Kobayashi, R. Murgia, A. D. Simone, V. Iršič, and M. Viel. Lyman- $\alpha$ constraints on ultralight scalar dark matter: Implications for the early and late universe. Physical Review D, 96(12), December 2017. doi: 10.1103/physrevd.96.123514.

A. Kogut, D. J. Fixsen, D. T. Chuss, J. Dotson, E. Dwek, M. Halpern, G. F. Hinshaw, S. M. Meyer, S. H. Moseley, M. D. Seiffert, D. N. Spergel, and E. J. Wollack. The Primordial Inflation Explorer (PIXIE): a nulling polarimeter for cosmic microwave background observations. Journal of Cosmology and Astroparticle Physics, 2011(07):025-025, July 2011. doi: 10.1088/1475-7516/2011/07/025.

M. Kopp, K. Vattis, and C. Skordis. Solving the Vlasov equation in two spatial dimensions with the Schrödinger method. Physical Review D, 96(12), December 2017. doi: 10.1103/ physrevd.96.123532.

J. Kormendy and K. C. Freeman. Scaling Laws for Dark Matter Halos in Late-Type and Dwarf Spheroidal Galaxies. In Dark Matter in Galaxies, volume 220 of IAU Symposium, page 377, July 2004. arXiv: astro-ph/0407321.

M. Kreuzer and H. Skarke. Reflexive polyhedra, weights and toric Calabi-Yau fibrations. Reviews in Mathematical Physics, 14:343-374, 2002. arXiv: math/0001106.

M. Kuhlen and C.-A. Faucher-Giguère. Concordance models of reionization: implications for faint galaxies and escape fraction evolution. Monthly Notices of the Royal Astronomical Society, 423(1):862-876, April 2012. doi: 10.1111/j.1365-2966.2012.20924.x.

C. Lacey and S. Cole. Merger rates in hierarchical models of galaxy formation. Monthly Notices of the Royal Astronomical Society, 262(3):627-649, June 1993. doi: 10.1093/ mnras/262.3.627.

D. Lam, T. Broadhurst, J. M. Diego, J. Lim, D. Coe, H. C. Ford, and W. Zheng. A Rigorous Free-form Lens Model of A2744 to Meet the Hubble Frontier Fields Challenge. The Astrophysical Journal, 797(2):98, December 2014. doi: 10.1088/0004-637x/797/2/98.

J.-W. Lee, J. Lee, and H.-C. Kim. The M-sigma Relation of Super Massive Black Holes from the Scalar Field Dark Matter. arXiv, December 2015. 1512.02351.

J. Lesgourgues. The Cosmic Linear Anisotropy Solving System (CLASS) I: Overview. arXiv, April 2011. 1104.2932.

E. Leung, T. Broadhurst, J. Lim, J. M. Diego, T. Chiueh, H.-Y. Schive, and R. Windhorst. Magnification Bias of Distant Galaxies in the Hubble Frontier Fields: Testing Wave Versus Particle Dark Matter Predictions. The Astrophysical Journal, 862(2):156, August 2018. doi: 10.3847/1538-4357/aacdad. 
D. G. Levkov, A. G. Panin, and I. I. Tkachev. Bose Condensation by Gravitational Interactions. arXiv, April 2018. 1804.05857.

D. G. Levkov, A. G. Panin, and I. I. Tkachev. Relativistic Axions from Collapsing Bose Stars. Physical Review Letters, 118(1), January 2017. doi: 10.1103/physrevlett.118. 011301.

A. Lewis and S. Bridle. Cosmological parameters from CMB and other data: A Monte Carlo approach. Physical Review D, 66(10), November 2002. doi: 10.1103/physrevd.66.103511.

B. Li, T. Rindler-Daller, and P. R. Shapiro. Cosmological constraints on Bose-Einsteincondensed scalar field dark matter. Physical Review D, 89(8), April 2014. doi: 10.1103/ physrevd.89.083536.

A. Lidz and L. Hui. Implications of a prereionization 21-cm absorption signal for fuzzy dark matter. Physical Review D, 98(2), July 2018. doi: 10.1103/physrevd.98.023011.

A. Lidz, O. Zahn, M. McQuinn, M. Zaldarriaga, and L. Hernquist. Detecting the Rise and Fall of $21 \mathrm{~cm}$ Fluctuations with the Murchison Widefield Array. The Astrophysical Journal, 680(2):962-974, June 2008. doi: 10.1086/587618.

S. L. Liebling and C. Palenzuela. Dynamical boson stars. Living Reviews in Relativity, 20 (1), November 2017. doi: 10.1007/s41114-017-0007-y.

H. W. Lin and A. Loeb. Scaling relations of halo cores for self-interacting dark matter. Journal of Cosmology and Astroparticle Physics, 2016(03):009-009, March 2016. doi: 10.1088/1475-7516/2016/03/009.

S.-C. Lin, H.-Y. Schive, S.-K. Wong, and T. Chiueh. Self-consistent construction of virialized wave dark matter halos. Physical Review D, 97(10), May 2018. doi: 10.1103/physrevd.97.103523.

A. Loeb and R. Barkana. The Reionization of the Universe by the First Stars and Quasars. Annual Review of Astronomy and Astrophysics, 39(1):19-66, September 2001. doi: 10. 1146/annurev.astro.39.1.19.

S. López, V. D’Odorico, S. L. Ellison, G. D. Becker, L. Christensen, G. Cupani, K. D. Denney, I. Pâris, G. Worseck, T. A. M. Berg, S. Cristiani, M. Dessauges-Zavadsky, M. Haehnelt, F. Hamann, J. Hennawi, V. Iršič, T.-S. Kim, P. López, R. Lund Saust, B. Ménard, S. Perrotta, J. X. Prochaska, R. Sánchez-Ramírez, M. Vestergaard, M. Viel, and L. Wisotzki. XQ-100: A legacy survey of one hundred $3.5 \lesssim z \lesssim 4.5$ quasars observed with VLT/X-shooter. Astronomy \& Astrophysics, 594:A91, October 2016. doi: 10.1051/ 0004-6361/201628161.

V. Lora, J. Magaña, A. Bernal, F. J. Sánchez-Salcedo, and E. K. Grebel. On the mass of ultra-light bosonic dark matter from galactic dynamics. Journal of Cosmology and 
Astroparticle Physics, 2012(02):011-011, February 2012. doi: 10.1088/1475-7516/2012/ $02 / 011$.

M. R. Lovell, C. S. Frenk, V. R. Eke, A. Jenkins, L. Gao, and T. Theuns. The properties of warm dark matter haloes. Monthly Notices of the Royal Astronomical Society, 439(1): 300-317, February 2014. doi: 10.1093/mnras/stt2431.

M. LoVerde. Halo bias in mixed dark matter cosmologies. Physical Review D, 90(8), October 2014. doi: 10.1103/physrevd.90.083530.

A. V. Macciò, S. Paduroiu, D. Anderhalden, A. Schneider, and B. Moore. Cores in warm dark matter haloes: a Catch 22 problem. Monthly Notices of the Royal Astronomical Society, 424:1105-1112, August 2012a. doi: 10.1111/j.1365-2966.2012.21284.x.

A. V. Macciò, S. Paduroiu, D. Anderhalden, A. Schneider, and B. Moore. Erratum: Cores in warm dark matter haloes: a Catch 22 problem. Monthly Notices of the Royal Astronomical Society, 428(4):3715-3716, November 2012b. doi: 10.1093/mnras/sts251.

P. Madau. Constraints on early star formation from the 21-cm global signal. Monthly Notices of the Royal Astronomical Society: Letters, 480(1):L43-L47, July 2018. doi: $10.1093 / \mathrm{mnrasl} / \mathrm{sly} 125$.

P. Madau, A. Meiksin, and M. J. Rees. 21 Centimeter Tomography of the Intergalactic Medium at High Redshift. The Astrophysical Journal, 475(2):429-444, February 1997. doi: $10.1086 / 303549$.

P. Madau, F. Haardt, and M. J. Rees. Radiative Transfer in a Clumpy Universe. III. The Nature of Cosmological Ionizing Sources. The Astrophysical Journal, 514(2):648-659, April 1999. doi: 10.1086/306975.

P. Madau, S. Shen, and F. Governato. Dark Matter Heating and Early Core Formation in Dwarf Galaxies. The Astrophysical Journal, 789(1):L17, June 2014. doi: 10.1088/ 2041-8205/789/1/117.

E. Madelung. Quantentheorie in hydrodynamischer Form. Zeitschrift für Physik, 40(3-4): 322-326, 1927. ISSN 0044-3328. doi: 10.1007/BF01400372.

S. Majumdar, S. Bharadwaj, and T. R. Choudhury. The effect of peculiar velocities on the epoch of reionization 21-cm signal. Monthly Notices of the Royal Astronomical Society, 434(3):1978-1988, July 2013. doi: 10.1093/mnras/stt1144.

D. J. E. Marsh. WarmAndFuzzy: the halo model beyond CDM. arXiv, May 2016a. 1605.05973 .

D. J. E. Marsh. Axion cosmology. Physics Reports, 643:1-79, July 2016b. doi: 10.1016/j. physrep.2016.06.005. 
D. J. E. Marsh and P. G. Ferreira. Ultralight scalar fields and the growth of structure in the Universe. Physical Review D, 82(10), November 2010. doi: 10.1103/physrevd.82.103528.

D. J. E. Marsh and A.-R. Pop. Axion dark matter, solitons and the cusp-core problem. Monthly Notices of the Royal Astronomical Society, 451(3):2479-2492, June 2015. doi: $10.1093 / \mathrm{mnras} / \mathrm{stv} 1050$.

D. J. E. Marsh and J. Silk. A model for halo formation with axion mixed dark matter. Monthly Notices of the Royal Astronomical Society, 437(3):2652-2663, November 2013. doi: $10.1093 / \mathrm{mnras} / \mathrm{stt} 2079$.

D. J. E. Marsh, E. Macaulay, M. Trebitsch, and P. G. Ferreira. Ultralight axions: Degeneracies with massive neutrinos and forecasts for future cosmological observations. Phys. Rev. D, 85(10):103514, May 2012. doi: 10.1103/PhysRevD.85.103514.

D. J. E. Marsh. Nonlinear hydrodynamics of axion dark matter: Relative velocity effects and quantum forces. Physical Review D, 91(12), June 2015. doi: 10.1103/physrevd.91. 123520 .

I. De Martino, T. Broadhurst, S.-H. H. Tye, T. Chiueh, H.-Y. Schive, and R. Lazkoz. Recognizing Axionic Dark Matter by Compton and de Broglie Scale Modulation of Pulsar Timing. Physical Review Letters, 119(22), November 2017. doi: 10.1103/physrevlett. 119.221103.

I. De Martino, T. Broadhurst, S.-H. H. Tye, T. Chiueh, and H.-Y. Schive. Dynamical Evidence of a Solitonic Core of $10^{9} M_{\odot}$ in the Milky Way. arXiv, 2018. 1807.08153.

S. Mashchenko, J. Wadsley, and H. M. P. Couchman. Stellar Feedback in Dwarf Galaxy Formation. Science, 319(5860):174-177, January 2008. doi: 10.1126/science.1148666.

C. A. Mason, M. Trenti, and T. Treu. The Galaxy UV Luminosity Function Before the Epoch of Reionization. The Astrophysical Journal, 813(1):21, October 2015. doi: 10. 1088/0004-637x/813/1/21.

S. McAlpine, J. C. Helly, M. Schaller, J. W. Trayford, Y. Qu, M. Furlong, R. G. Bower, R. A. Crain, J. Schaye, T. Theuns, C. D. Vecchia, C. S. Frenk, I. G. McCarthy, A. Jenkins, Y. Rosas-Guevara, S. D. M. White, M. Baes, P. Camps, and G. Lemson. The eagle simulations of galaxy formation: Public release of halo and galaxy catalogues. Astronomy and Computing, 15:72-89, April 2016. doi: 10.1016/j.ascom.2016.02.004.

S. S. McGaugh, V. C. Rubin, and W. J. G. de Blok. High-Resolution Rotation Curves of Low Surface Brightness Galaxies. I. Data. The Astronomical Journal, 122(5):2381-2395, November 2001. doi: 10.1086/323448.

I. D. McGreer, A. Mesinger, and X. Fan. The first (nearly) model-independent constraint on the neutral hydrogen fraction at . Monthly Notices of the Royal Astronomical Society, 415(4):3237-3246, July 2011. doi: 10.1111/j.1365-2966.2011.18935.x. 
I. D. McGreer, A. Mesinger, and V. D'Odorico. Model-independent evidence in favour of an end to reionization by $\mathrm{z} \approx 6$. Monthly Notices of the Royal Astronomical Society, 447 (1):499-505, December 2014. doi: 10.1093/mnras/stu2449.

N. Menci, A. Merle, M. Totzauer, A. Schneider, A. Grazian, M. Castellano, and N. G. Sanchez. Fundamental Physics with the Hubble Frontier Fields: Constraining Dark Matter Models with the Abundance of Extremely Faint and Distant Galaxies. The Astrophysical Journal, 836(1):61, February 2017. doi: 10.3847/1538-4357/836/1/61.

R. A. Meyer, S. E. I. Bosman, K. Kakiichi, and R. S. Ellis. The role of galaxies and AGN in reionizing the IGM - II: metal-tracing the faint sources of reionization at $5 \lesssim z \lesssim 6$. arXiv, 2018. 1807.07899.

F. Michel and I. G. Moss. Relativistic collapse of axion stars. Physics Letters B, 785:9-13, October 2018. doi: 10.1016/j.physletb.2018.07.063.

D. Minniti. Field Stars and Clusters of the Galactic Bulge: Implications for Galaxy Formation. The Astrophysical Journal, 459:175, March 1996. doi: 10.1086/176879.

H. Mo, F. C. van den Bosch, and S. White. Galaxy Formation and Evolution. Cambridge University Press, May 2010. ISBN 9780521857932.

P. Mocz and S. Succi. Numerical solution of the nonlinear Schrödinger equation using smoothed-particle hydrodynamics. Physical Review E, 91(5), May 2015. doi: 10.1103/ physreve.91.053304.

P. Mocz, M. Vogelsberger, V. H. Robles, J. Zavala, M. Boylan-Kolchin, A. Fialkov, and L. Hernquist. Galaxy formation with BECDM - I. Turbulence and relaxation of idealized haloes. Monthly Notices of the Royal Astronomical Society, 471(4):4559-4570, July 2017. doi: $10.1093 /$ mnras/stx1887.

P. Mocz, L. Lancaster, A. Fialkov, F. Becerra, and P.-H. Chavanis. SchrödingerPoisson-Vlasov-Poisson correspondence. Physical Review D, 97(8), April 2018. doi: 10.1103/physrevd.97.083519.

J. J. Monaghan. Smoothed Particle Hydrodynamics. Annual Review of Astronomy and Astrophysics, 30(1):543-574, September 1992. doi: 10.1146/annurev.aa.30.090192.002551.

B. Moore. Evidence against dissipation-less dark matter from observations of galaxy haloes. Nature, 370(6491):629-631, August 1994. doi: 10.1038/370629a0.

B. Moore, S. Ghigna, F. Governato, G. Lake, T. Quinn, J. Stadel, and P. Tozzi. Dark Matter Substructure within Galactic Halos. The Astrophysical Journal, 524(1):L19-L22, October 1999. doi: 10.1086/312287. 
NANOGrav Collaboration. The NANOGrav 11 Year Data Set: Pulsar-timing Constraints on the Stochastic Gravitational-wave Background. The Astrophysical Journal, 859(1): 47, May 2018. doi: 10.3847/1538-4357/aabd3b.

M. Naraschewski, H. Wallis, A. Schenzle, J. I. Cirac, and P. Zoller. Interference of Bose condensates. Physical Review A, 54(3):2185-2196, September 1996. doi: 10.1103/physreva.54.2185.

J. F. Navarro, A. Ludlow, V. Springel, J. Wang, M. Vogelsberger, S. D. M. White, A. Jenkins, C. S. Frenk, and A. Helmi. The diversity and similarity of simulated cold dark matter haloes. Monthly Notices of the Royal Astronomical Society, 402(1):21-34, December 2009. doi: 10.1111/j.1365-2966.2009.15878.x.

M. Ness, K. Freeman, E. Athanassoula, E. Wylie de Boer, J. Bland-Hawthorn, M. Asplund, G. F. Lewis, D. Yong, R. R. Lane, L. L. Kiss, and R. Ibata. ARGOS - IV. The kinematics of the Milky Way bulge. Monthly Notices of the Royal Astronomical Society, 432(3): 2092-2103, May 2013. doi: 10.1093/mnras/stt533.

M. Nori and M. Baldi. AX-GADGET: a new code for cosmological simulations of Fuzzy Dark Matter and Axion models. Monthly Notices of the Royal Astronomical Society, 478 (3):3935-3951, May 2018. doi: 10.1093/mnras/sty1224.

P. Noterdaeme, P. Petitjean, C. Ledoux, and R. Srianand. Evolution of the cosmological mass density of neutral gas from Sloan Digital Sky Survey II - Data Release 7. Astronomy 86 Astrophysics, 505(3):1087-1098, August 2009. doi: 10.1051/0004-6361/200912768.

P. Noterdaeme, P. Petitjean, W. C. Carithers, I. Pâris, A. Font-Ribera, S. Bailey, E. Aubourg, D. Bizyaev, G. Ebelke, H. Finley, J. Ge, E. Malanushenko, V. Malanushenko, J. Miralda-Escudé, A. D. Myers, D. Oravetz, K. Pan, M. M. Pieri, N. P. Ross, D. P. Schneider, A. Simmons, and D. G. York. Column density distribution and cosmological mass density of neutral gas: Sloan Digital Sky Survey-III Data Release 9. Astronomy \& Astrophysics, 547:L1, October 2012. doi: 10.1051/0004-6361/201220259.

P. A. Oesch, R. J. Bouwens, G. D. Illingworth, C. M. Carollo, M. Franx, I. Labbé, D. Magee, M. Stiavelli, M. Trenti, and P. G. van Dokkum. z 7 Galaxies in the HUDF: First Epoch WFC3/IR Results. The Astrophysical Journal, 709(1):L16-L20, December 2009. doi: 10.1088/2041-8205/709/1/116.

P. A. Oesch, G. Brammer, P. G. van Dokkum, G. D. Illingworth, R. J. Bouwens, I. Labbé, M. Franx, I. Momcheva, M. L. N. Ashby, G. G. Fazio, V. Gonzalez, B. Holden, D. Magee, R. E. Skelton, R. Smit, L. R. Spitler, M. Trenti, and S. P. Willner. A Remarkably Luminous Galaxy at $\mathrm{z}=11.1$ Measured with Hubble Space Telescope Grism Spectroscopy. The Astrophysical Journal, 819(2):129, March 2016. doi: 10.3847/0004-637x/819/2/129.

K. A. Oman, J. F. Navarro, A. Fattahi, C. S. Frenk, T. Sawala, S. D. M. White, R. Bower, R. A. Crain, M. Furlong, M. Schaller, J. Schaye, and T. Theuns. The unexpected 
diversity of dwarf galaxy rotation curves. Monthly Notices of the Royal Astronomical Society, 452(4):3650-3665, August 2015. doi: 10.1093/mnras/stv1504.

J. Oñorbe, M. Boylan-Kolchin, J. S. Bullock, P. F. Hopkins, D. Kereš, C.-A. FaucherGiguère, E. Quataert, and N. Murray. Forged in FIRE: cusps, cores and baryons in low-mass dwarf galaxies. Monthly Notices of the Royal Astronomical Society, 454(2): 2092-2106, October 2015. doi: 10.1093/mnras/stv2072.

N. Palanque-Delabrouille, C. Yèche, A. Borde, J.-M. Le Goff, G. Rossi, M. Viel, É. Aubourg, S. Bailey, J. Bautista, M. Blomqvist, A. Bolton, J. S. Bolton, N. G. Busca, B. Carithers, R. A. C. Croft, K. S. Dawson, T. Delubac, A. Font-Ribera, S. Ho, D. Kirkby, K.-G. Lee, D. Margala, J. Miralda-Escudé, D. Muna, A. D. Myers, P. Noterdaeme, I. Pâris, P. Petitjean, M. M. Pieri, J. Rich, E. Rollinde, N. P. Ross, D. J. Schlegel, D. P. Schneider, A. Slosar, and D. H. Weinberg. The one-dimensional Lyaforest power spectrum from BOSS. Astronomy \& Astrophysics, 559:A85, November 2013. doi: 10.1051/0004-6361/201322130.

C. Palenzuela, P. Pani, M. Bezares, V. Cardoso, L. Lehner, and S. Liebling. Gravitational wave signatures of highly compact boson star binaries. Physical Review D, 96(10), November 2017. doi: 10.1103/physrevd.96.104058.

A. Paredes and H. Michinel. Interference of dark matter solitons and galactic offsets. Physics of the Dark Universe, 12:50-55, June 2016. doi: 10.1016/j.dark.2016.02.003.

R. D. Peccei and H. R. Quinn. CP Conservation in the Presence of Pseudoparticles. Physical Review Letters, 38(25):1440-1443, June 1977. doi: 10.1103/physrevlett.38.1440.

R. Penrose and R. M. Floyd. Extraction of Rotational Energy from a Black Hole. Nature Physical Science, 229(6):177-179, February 1971. doi: 10.1038/physci229177a0.

C. Péroux, R. G. McMahon, L. J. Storrie-Lombardi, and M. J. Irwin. The evolution of $\Omega \mathrm{HI}$ and the epoch of formation of damped Lyman $\alpha$ absorbers. Monthly Notices of the Royal Astronomical Society, 346(4):1103-1115, December 2003. doi: 10.1111/j.1365-2966.2003. 07129.x.

Planck Collaboration. Planck2013 results. I. Overview of products and scientific results. Astronomy 85 Astrophysics, 571:A1, October 2014. doi: 10.1051/0004-6361/201321529.

Planck Collaboration. Planck intermediate results. Astronomy \& 8 Astrophysics, 596:A108, December 2016a. doi: 10.1051/0004-6361/201628897.

Planck Collaboration. Planck 2015 results. Astronomy \& Astrophysics, 594:A11, September 2016b. doi: 10.1051/0004-6361/201526926.

Planck Collaboration. Planck 2018 results. VI. Cosmological parameters. arXiv, July 2018. 1807.06209. 
J. C. Pober, Z. S. Ali, A. R. Parsons, M. McQuinn, J. E. Aguirre, G. Bernardi, R. F. Bradley, C. L. Carilli, C. Cheng, D. R. DeBoer, M. R. Dexter, S. R. Furlanetto, J. Grobbelaar, J. Horrell, D. C. Jacobs, P. J. Klima, S. A. Kohn, A. Liu, D. H. E. MacMahon, M. Maree, A. Mesinger, D. F. Moore, N. Razavi-Ghods, I. I. Stefan, W. P. Walbrugh, A. Walker, and H. Zheng. PAPER-64 Constraints On Reionization II: The Temperature Of The $\mathrm{z}=8.4$ Intergalactic Medium. The Astrophysical Journal, 809(1): 62, August 2015. doi: 10.1088/0004-637x/809/1/62.

A. Pontzen and F. Governato. Cold dark matter heats up. Nature, 506:171-178, February 2014. doi: $10.1038 /$ nature12953.

A. Pontzen, F. Governato, M. Pettini, C. M. Booth, G. Stinson, J. Wadsley, A. Brooks, T. Quinn, and M. Haehnelt. Damped Lyman $\alpha$ systems in galaxy formation simulations. Monthly Notices of the Royal Astronomical Society, October 2008. doi: 10.1111/j.1365-2966.2008.13782.x.

M. M. Popov. A new method of computation of wave fields using Gaussian beams. Wave Motion, 4(1):85-97, January 1982. doi: 10.1016/0165-2125(82)90016-6.

N. K. Porayko and K. A. Postnov. Constraints on ultralight scalar dark matter from pulsar timing. Physical Review D, 90(6), September 2014. doi: 10.1103/physrevd.90.062008.

W. H. Press and P. Schechter. Formation of Galaxies and Clusters of Galaxies by SelfSimilar Gravitational Condensation. The Astrophysical Journal, 187:425, February 1974. doi: $10.1086 / 152650$.

W. H. Press, B. S. Ryden, and D. N. Spergel. Single mechanism for generating large-scale structure and providing dark missing matter. Physical Review Letters, 64(10):1084-1087, March 1990. doi: 10.1103/physrevlett.64.1084.

W. H. Press, S. A. Teukolsky, W. T. Vetterling, and B. P. Flannery. Numerical Recipes 3rd Edition: The Art of Scientific Computing. Cambridge University Press, New York, NY, USA, 3 edition, 2007. ISBN 0521880688, 9780521880688.

J. R. Pritchard and S. R. Furlanetto. Descending from on high: Lyman-series cascades and spin-kinetic temperature coupling in the 21-cm line. Monthly Notices of the Royal Astronomical Society, 367(3):1057-1066, April 2006. doi: 10.1111/j.1365-2966.2006.10028.x.

J. R. Pritchard and A. Loeb. $21 \mathrm{~cm}$ cosmology in the 21st century. Reports on Progress in Physics, 75(8):086901, July 2012. doi: 10.1088/0034-4885/75/8/086901.

J. Ralston. Gaussian beams and the propagation of singularities. MAA Studies in Mathematics, 23:206-248, 1982.

J. I. Read and D. Erkal. Abundance matching with the mean star formation rate: there is no missing satellites problem in the Milky Way. arXiv, July 2018. 
M. J. Rees and J. P. Ostriker. Cooling, dynamics and fragmentation of massive gas clouds: clues to the masses and radii of galaxies and clusters. Monthly Notices of the Royal Astronomical Society, 179(4):541-559, August 1977. doi: 10.1093/mnras/179.4.541.

R. M. Rich, D. B. Reitzel, C. D. Howard, and H. S. Zhao. The Bulge Radial Velocity Assay: Techniques and a Rotation Curve. The Astrophysical Journal, 658(1):L29-L32, March 2007. doi: 10.1086/513509.

T. Richardson and M. Fairbairn. On the dark matter profile in Sculptor: breaking the $\beta$ degeneracy with Virial shape parameters. Monthly Notices of the Royal Astronomical Society, 441(2):1584-1600, May 2014. doi: 10.1093/mnras/stu691.

T. Rindler-Daller and P. R. Shapiro. Vortices and Angular Momentum in Bose-EinsteinCondensed Cold Dark Matter Halos. arXiv, December 2009. 0912.2897.

T. Rindler-Daller and P. R. Shapiro. Angular momentum and vortex formation in BoseEinstein-condensed cold dark matter haloes. Monthly Notices of the Royal Astronomical Society, 422(1):135-161, March 2012. doi: 10.1111/j.1365-2966.2012.20588.x.

T. Rindler-Daller and P. R. Shapiro. Finding New Signature Effects on Galactic Dynamics to Constrain Bose-Einstein-Condensed Cold Dark Matter. In Astrophysics and Space Science Proceedings, pages 163-182. Springer International Publishing, November 2013. doi: 10.1007/978-3-319-02063-1_12.

T. Rindler-Daller and P. R. Shapiro. Complex scalar field dark matter on galactic scales. Modern Physics Letters A, 29(02):1430002, January 2014. doi: 10.1142/ s021773231430002x.

B. E. Robertson, S. R. Furlanetto, E. Schneider, S.Charlot, R. S. Ellis, D. P. Stark, R. J. McLure, J. S. Dunlop, A. Koekemoer, M. A. Schenker, M. Ouchi, Y. Ono, E. CurtisLake, A. B. Rogers, R. A. A. Bowler, and M. Cirasuolo. New Constraints on Cosmic Reionization from the 2012 Hubble Ultra Deep Field Campaign. The Astrophysical Journal, 768(1):71, April 2013. doi: 10.1088/0004-637x/768/1/71.

B. E. Robertson, R. S. Ellis, S. R. Furlanetto, and J. S. Dunlop. Cosmic Reionization and Early Star-forming Galaxies: A Joint Analysis of New Constraints from Planck and the Hubble Space Telescope. The Astrophysical Journal, 802(2):L19, April 2015. doi: 10.1088/2041-8205/802/2/119.

É. Roche. La figure d'une masse fluide soumise à l'attraction d'un point éloigné. Academie des sciences de Montpellier, 1:243, 1850.

A. Röhrl, M. Naraschewski, A. Schenzle, and H. Wallis. Transition from Phase Locking to the Interference of Independent Bose Condensates: Theory versus Experiment. Physical Review Letters, 78(22):4143-4146, June 1997. doi: 10.1103/physrevlett.78.4143. 
R. Ruffini and S. Bonazzola. Systems of Self-Gravitating Particles in General Relativity and the Concept of an Equation of State. Physical Review, 187(5):1767-1783, November 1969. doi: 10.1103/physrev.187.1767.

N. Sanchis-Gual, C. Herdeiro, E. Radu, J. C. Degollado, and J. A. Font. Numerical evolutions of spherical Proca stars. Physical Review D, 95(10), May 2017. doi: 10.1103/ physrevd.95.104028.

N. Sanchis-Gual, C. Herdeiro, J. A. Font, and E. Radu. Head-on collisions of Proca stars. arXiv, June 2018. 1806.07779.

A. Sarkar, R. Mondal, S. Das, S. K. Sethi, S. Bharadwaj, and D. J. E. Marsh. The effects of the small-scale DM power on the cosmological neutral hydrogen (HI) distribution at high redshifts. Journal of Cosmology and Astroparticle Physics, 2016(04):012-012, April 2016. doi: 10.1088/1475-7516/2016/04/012.

A. Sarkar, S. K. Sethi, and S. Das. The effects of the small-scale behaviour of dark matter power spectrum on CMB spectral distortion. Journal of Cosmology and Astroparticle Physics, 2017(07):012-012, July 2017. doi: 10.1088/1475-7516/2017/07/012.

T. Sawala, C. S. Frenk, A. Fattahi, J. F. Navarro, R. G. Bower, R. A. Crain, C. D. Vecchia, M. Furlong, J. C. Helly, A. Jenkins, K. A. Oman, M. Schaller, J. Schaye, T. Theuns, J. Trayford, and S. D. M. White. The APOSTLE simulations: solutions to the Local Group's cosmic puzzles. Monthly Notices of the Royal Astronomical Society, 457(2): 1931-1943, February 2016. doi: 10.1093/mnras/stw145.

M. Schaller, C. Becker, O. Ruchayskiy, A. Boyarsky, and M. Shaposhnikov. A new framework for numerical simulations of structure formation. Monthly Notices of the Royal Astronomical Society, 442(4):3073-3095, June 2014. doi: 10.1093/mnras/stu1069.

P. Schechter. An analytic expression for the luminosity function for galaxies. The Astrophysical Journal, 203:297, January 1976. doi: 10.1086/154079.

H.-Y. Schive and T. Chiueh. Halo abundance and assembly history with extreme-axion wave dark matter at $\mathrm{z} \geq 4$. Monthly Notices of the Royal Astronomical Society: Letters, 473(1):L36-L40, October 2017. doi: 10.1093/mnrasl/slx159.

H.-Y. Schive, T. Chiueh, and T. Broadhurst. Cosmic structure as the quantum interference of a coherent dark wave. Nature Physics, 10:496-499, July 2014a. doi: 10.1038/nphys2996.

H.-Y. Schive, M.-H. Liao, T.-P. Woo, S.-K. Wong, T. Chiueh, T. Broadhurst, and W.Y. P. Hwang. Understanding the Core-Halo Relation of Quantum Wave Dark Matter from 3D Simulations. Physical Review Letters, 113(26):261302, December 2014b. doi: 10.1103/PhysRevLett.113.261302. 
H.-Y. Schive, T. Chiueh, T. Broadhurst, and K.-W. Huang. Contrasting Galaxy Formation from Quantum Wave Dark Matter, $\psi \mathrm{DM}$, with $\Lambda \mathrm{CDM}$, using Planck and Hubble Data. The Astrophysical Journal, 818(1):89, February 2016. doi: 10.3847/0004-637X/818/1/ 89.

A. Schneider. Constraining Non-Cold Dark Matter Models with the Global 21-cm Signal. arXiv, April 2018. 1805.00021.

A. Schneider, R. E. Smith, A. V. Macciò, and B. Moore. Non-linear evolution of cosmological structures in warm dark matter models. Monthly Notices of the Royal Astronomical Society, 424(1):684-698, June 2012. doi: 10.1111/j.1365-2966.2012.21252.x.

A. Schneider, D. Anderhalden, A. V. Macciò, and J. Diemand. Warm dark matter does not do better than cold dark matter in solving small-scale inconsistencies. Monthly Notices of the Royal Astronomical Society: Letters, 441(1):L6-L10, March 2014. doi: $10.1093 / \mathrm{mnrasl} / \mathrm{slu} 034$.

J. Schroeder, A. Mesinger, and Z. Haiman. Evidence of Gunn-Peterson damping wings in high-z quasar spectra: strengthening the case for incomplete reionization at $\mathrm{z} \sim 6-7$. Monthly Notices of the Royal Astronomical Society, 428(4):3058-3071, November 2012. doi: $10.1093 / \mathrm{mnras} / \mathrm{sts} 253$.

C. Schultz, J. Oñorbe, K. N. Abazajian, and J. S. Bullock. The high-z universe confronts warm dark matter: Galaxy counts, reionization and the nature of dark matter. Monthly Notices of the Royal Astronomical Society, 442(2):1597-1609, June 2014. doi: 10.1093/ mnras/stu976.

B. Schwabe, J. C. Niemeyer, and J. F. Engels. Simulations of solitonic core mergers in ultralight axion dark matter cosmologies. Physical Review D, 94(4), August 2016. doi: 10.1103/physrevd.94.043513.

SDSS Collaboration. The Baryon Oscillation Spectroscopic Survey of SDSS-III. The Astronomical Journal, 145(1):10, December 2012. doi: 10.1088/0004-6256/145/1/10.

E. Seidel and W.-M. Suen. Dynamical evolution of boson stars: Perturbing the ground state. Physical Review D, 42(2):384-403, July 1990. doi: 10.1103/physrevd.42.384.

E. Seidel and W.-M. Suen. Formation of solitonic stars through gravitational cooling. Physical Review Letters, 72(16):2516-2519, April 1994. doi: 10.1103/physrevlett.72.2516.

P. R. Shapiro and I. T. Iliev. The Central Mass and Phase-Space Densities of Dark Matter Halos: Cosmological Implications. The Astrophysical Journal, 565(1):L1-L4, January 2002. doi: $10.1086 / 339243$.

R. K. Sheth and G. Tormen. Large-scale bias and the peak background split. Monthly Notices of the Royal Astronomical Society, 308(1):119-126, September 1999. doi: 10. 1046/j.1365-8711.1999.02692.x. 
P. Sikivie. Caustic rings of dark matter. Physics Letters B, 432(1-2):139-144, July 1998. doi: 10.1016/s0370-2693(98)00595-4.

J. D. Simon, A. D. Bolatto, A. Leroy, L. Blitz, and E. L. Gates. High-Resolution Measurements of the Halos of Four Dark Matter-Dominated Galaxies: Deviations from a Universal Density Profile. The Astrophysical Journal, 621(2):757-776, March 2005. doi: $10.1086 / 427684$.

A. Songaila and L. L. Cowie. The Evolution of Lyman Limit Absorption Systems to Redshift Six. The Astrophysical Journal, 721(2):1448-1466, September 2010. doi: 10. 1088/0004-637x/721/2/1448.

M. Spano, M. Marcelin, P. Amram, C. Carignan, B. Epinat, and O. Hernandez. GHASP: an $\mathrm{H} \alpha$ kinematic survey of spiral and irregular galaxies - V. Dark matter distribution in 36 nearby spiral galaxies. Monthly Notices of the Royal Astronomical Society, 383(1): 297-316, December 2007. doi: 10.1111/j.1365-2966.2007.12545.x.

D. N. Spergel and P. J. Steinhardt. Observational Evidence for Self-Interacting Cold Dark Matter. Physical Review Letters, 84(17):3760-3763, April 2000. doi: 10.1103/physrevlett. 84.3760 .

E. A. Spiegel. Fluid dynamical form of the linear and nonlinear Schrödinger equations. Physica D: Nonlinear Phenomena, 1(2):236-240, June 1980. doi: 10.1016/0167-2789(80) 90015-9.

V. Springel. The cosmological simulation code gadget-2. Monthly Notices of the Royal Astronomical Society, 364(4):1105-1134, December 2005. doi: 10.1111/j.1365-2966.2005. 09655.x.

V. Springel, J. Wang, M. Vogelsberger, A. Ludlow, A. Jenkins, A. Helmi, J. F. Navarro, C. S. Frenk, and S. D. M. White. The Aquarius Project: the subhaloes of galactic haloes. Monthly Notices of the Royal Astronomical Society, 391(4):1685-1711, December 2008. doi: 10.1111/j.1365-2966.2008.14066.x.

V. Springel, R. Pakmor, A. Pillepich, R. Weinberger, D. Nelson, L. Hernquist, M. Vogelsberger, S. Genel, P. Torrey, F. Marinacci, and J. Naiman. First results from the IllustrisTNG simulations: matter and galaxy clustering. Monthly Notices of the Royal Astronomical Society, 475(1):676-698, December 2017. doi: 10.1093/mnras/stx3304.

A. A. Starobinsky. Amplification of waves reflected from a rotating "black hole". Soviet Physics JETP, 37(1):28-32, July 1973.

G. Steigman. Primordial Nucleosynthesis in the Precision Cosmology Era. Annual Review of Nuclear and Particle Science, 57(1):463-491, November 2007. doi: 10.1146/annurev. nucl.56.080805.140437. 
M. J. Stott and D. J. E. Marsh. Black Hole Spin Constraints on the Mass Spectrum and Number of Axion-like Fields. arXiv, May 2018. 1805.02016.

L. E. Strigari, C. S. Frenk, and S. D. M. White. Dynamical models for the Sculptor dwarf spheroidal in a Lambda CDM universe. arXiv, June 2014. 1406.6079.

A. Suárez and P.-H. Chavanis. Hydrodynamic representation of the Klein-Gordon-Einstein equations in the weak field limit: General formalism and perturbations analysis. Physical Review D, 92(2), July 2015. doi: 10.1103/physrevd.92.023510.

P. Svrcek. Cosmological Constant and Axions in String Theory. arXiv, July 2006. hepth/0607086.

P. Svrcek and E. Witten. Axions in String Theory. Journal of High Energy Physics, 2006 (06):051-051, June 2006. doi: 10.1088/1126-6708/2006/06/051.

T. Swart and V. Rousse. A Mathematical Justification for the Herman-Kluk Propagator. arXiv, December 2007. 0712.0752.

I. Szapudi and N. Kaiser. Cosmological Perturbation Theory Using the Schrödinger Equation. The Astrophysical Journal, 583(1):L1-L4, January 2003. doi: 10.1086/368013.

K. Takahashi. Distribution Functions in Classical and Quantum Mechanics. Progress of Theoretical Physics Supplement, 98:109-156, 1989. doi: 10.1143/ptps.98.109.

S. A. Teukolsky. Rotating Black Holes: Separable Wave Equations for Gravitational and Electromagnetic Perturbations. Physical Review Letters, 29(16):1114-1118, October 1972. doi: 10.1103/physrevlett.29.1114.

MAGIC AGILE ASAS-SN HAWC H.E.S.S. INTEGRAL Kanata Kiso Kapteyn Liverpool Telescope Subaru Swift/NuSTAR VERITAS VLA/17B-403 teams The IceCube Collaboration, Fermi-LAT. Multimessenger observations of a flaring blazar coincident with high-energy neutrino IceCube-170922A. Science, page eaat1378, July 2018. doi: 10.1126/science.aat1378.

The LIGO Scientific Collaboration and The Virgo Collaboration. GW170817: Observation of Gravitational Waves from a Binary Neutron Star Inspiral. Physical Review Letters, 119(16), October 2017. doi: 10.1103/physrevlett.119.161101.

E. Tollet, A. V. Macciò, A. A. Dutton, G. S. Stinson, L. Wang, C. Penzo, T. A. Gutcke, T. Buck, X. Kang, C. Brook, A. Di Cintio, B. W. Keller, and J. Wadsley. NIHAO IV: core creation and destruction in dark matter density profiles across cosmic time. Monthly Notices of the Royal Astronomical Society, 456(4):3542-3552, January 2016. doi: 10.1093/mnras/stv2856. 
C. J. Trahan and R. E. Wyatt. Quantum Dynamics with Trajectories: Introduction to Quantum Hydrodynamics. Interdisciplinary Applied Mathematics. Springer New York, 2005. ISBN 9780387229645.

M. J. Turk, B. D. Smith, J. S. Oishi, S. Skory, S. W. Skillman, T. Abel, and M. L. Norman. yt: A Multi-code Analysis Toolkit for Astrophysical Simulation Data. The Astrophysical Journal Supplement Series, 192(1):9, December 2010. doi: 10.1088/0067-0049/192/1/9.

M. S. Turner. Coherent scalar-field oscillations in an expanding universe. Physical Review D, 28(6):1243-1247, September 1983. doi: 10.1103/physrevd.28.1243.

C. Uhlemann. Finding closure: approximating Vlasov-Poisson using finitely generated cumulants. arXiv, July 2018. 1807.07274.

C. Uhlemann, M. Kopp, and T. Haugg. Schrödinger method as N-body double and UV completion of dust. Physical Review D, 90(2), July 2014. doi: 10.1103/physrevd.90. 023517 .

W. G. Unruh. Second quantization in the Kerr metric. Physical Review D, 10(10):31943205, November 1974. doi: 10.1103/physrevd.10.3194.

L. A. Ureña-López. Oscillatons revisited. Classical and Quantum Gravity, 19(10):26172632, April 2002. doi: 10.1088/0264-9381/19/10/307.

L. A. Ureña-López and A. X. Gonzalez-Morales. Towards accurate cosmological predictions for rapidly oscillating scalar fields as dark matter. Journal of Cosmology and Astroparticle Physics, 2016(07):048-048, July 2016. doi: 10.1088/1475-7516/2016/07/048.

L. A. Ureña-López, T. Matos, and R. Becerril. Inside oscillatons. Classical and Quantum Gravity, 19(23):6259-6277, November 2002. doi: 10.1088/0264-9381/19/23/320.

C. Vafa and E. Witten. Parity Conservation in Quantum Chromodynamics. Physical Review Letters, 53(6):535-536, August 1984. doi: 10.1103/physrevlett.53.535.

A. Vale and J. P. Ostriker. Linking halo mass to galaxy luminosity. Monthly Notices of the Royal Astronomical Society, 353(1):189-200, September 2004. doi: 10.1111/j.1365-2966. 2004.08059.x.

J. Veltmaat and J. C. Niemeyer. Cosmological particle-in-cell simulations with ultralight axion dark matter. Physical Review D, 94(12), December 2016. doi: 10.1103/physrevd. 94.123523 .

J. Veltmaat, J. C. Niemeyer, and B. Schwabe. Formation and structure of ultralight bosonic dark matter halos. Physical Review D, 98(4), August 2018. doi: 10.1103/physrevd.98. 043509 . 
J. Vicens, J. Salvado, and J. Miralda-Escud. Bosonic dark matter halos: excited states and relaxation in the potential of the ground state. arXiv, February 2018. 1802.10513.

M. Viel, J. Lesgourgues, M. G. Haehnelt, S. Matarrese, and A. Riotto. Constraining warm dark matter candidates including sterile neutrinos and light gravitinos with WMAP and the Lyman- $\alpha$ forest. Physical Review D, 71(6), March 2005. doi: 10.1103/physrevd.71. 063534 .

M. Viel, G. D. Becker, J. S. Bolton, and M. G. Haehnelt. Warm dark matter as a solution to the small scale crisis: New constraints from high redshift Lyman- $\alpha$ forest data. Physical Review D, 88(4), August 2013. doi: 10.1103/physrevd.88.043502.

L. Visinelli, S. Baum, J.Redondo, K. Freese, and F. Wilczek. Dilute and dense axion stars. Physics Letters B, 777:64-72, February 2018. doi: 10.1016/j.physletb.2017.12.010.

M. Vogelsberger, J. Zavala, and A. Loeb. Subhaloes in self-interacting galactic dark matter haloes. Monthly Notices of the Royal Astronomical Society, 423(4):3740-3752, May 2012. doi: $10.1111 / \mathrm{j} .1365-2966.2012 .21182 . x$.

R. M. Wald. Stimulated-emission effects in particle creation near black holes. Physical Review D, 13(12):3176-3182, June 1976. doi: 10.1103/physrevd.13.3176.

R. M. Wald. General Relativity. University Of Chicago Press, 1984. ISBN 0226870332.

M. G. Walker and J. Peñarrubia. A Method for Measuring (Slopes of) the Mass Profiles of Dwarf Spheroidal Galaxies. The Astrophysical Journal, 742(1):20, November 2011. doi: 10.1088/0004-637x/742/1/20.

M. G. Walker, M. Mateo, E. W. Olszewski, O. Y. Gnedin, X. Wang, B. Sen, and M. Woodroofe. Velocity Dispersion Profiles of Seven Dwarf Spheroidal Galaxies. The Astrophysical Journal, 667(1):L53-L56, September 2007. doi: 10.1086/521998.

M. G. Walker, M. Mateo, and E. W. Olszewski. Stellar Velocities in the Carina, Fornax, Sculptor and Sextans dSph Galaxies: Data from the Magellan/MMFS Survey. The Astronomical Journal, 137(2):3100-3108, January 2009. doi: 10.1088/0004-6256/137/2/ 3100 .

R. L. Webster. Results from the MWA EoR Experiment. Proceedings of the International Astronomical Union, 12(S333):77-82, October 2017. doi: 10.1017/s1743921318000893.

S. Weinberg. Gravitation and cosmology: principles and applications of the general theory of relativity. Wiley, 1972. ISBN 9780471925675.

A. R. Wetzel, P. F. Hopkins, J.-H. Kim, C.-A. Faucher-Gigure, D. Keres, and E. Quataert. Reconciling Dwarf Galaxies with $\Lambda \mathrm{CDM}$ Cosmology: Simulating a Realistic Population of Satellites around a Milky Waymass Galaxy. The Astrophysical Journal Letters, 827 (2):L23, August 2016. 
J. Y. Widdicombe, T. Helfer, D. J. E. Marsh, and E. A. Lim. Formation of Relativistic Axion Stars. arXiv, June 2018. 1806.09367.

L. M. Widrow and N. Kaiser. Using the Schroedinger Equation to Simulate Collisionless Matter. The Astrophysical Journal, 416:L71, October 1993. doi: 10.1086/187073.

WiggleZ Collaboration. The WiggleZ Dark Energy Survey: Final data release and cosmological results. Physical Review D, 86(10), November 2012. doi: 10.1103/physrevd.86. 103518.

E. Witten. Some properties of O(32) superstrings. Physics Letters B, 149(4-5):351-356, December 1984. doi: 10.1016/0370-2693(84)90422-2.

WMAP Collaboration. Nine-Year Wilkinson Microwave Anisotropy Probe (WMAP) Observations: Final Maps and Results. The Astrophysical Journal Supplement Series, 208 (2):20, September 2013. doi: 10.1088/0067-0049/208/2/20.

J. Wolf, G. D. Martinez, J. S. Bullock, M. Kaplinghat, M. Geha, R. R. Muñoz, J. D. Simon, and F. F. Avedo. Accurate masses for dispersion-supported galaxies. Monthly Notices of the Royal Astronomical Society, 406(2):1220-1237, May 2010. doi: 10.1111/ j.1365-2966.2010.16753.x.

T.-P. Woo and T. Chiueh. High-Resolution Simulation on Structure Formation with Extremely Light Bosonic Dark Matter. The Astrophysical Journal, 697(1):850-861, May 2009. doi: 10.1088/0004-637x/697/1/850.

S. A. Wouthuysen. On the excitation mechanism of the 21-cm (radio-frequency) interstellar hydrogen emission line. The Astronomical Journal, 57:31, 1952. doi: 10.1086/106661.

Y. Xu, B. Yue, and X. Chen. The Global $21 \mathrm{~cm}$ Absorption from Cosmic Dawn with Inhomogeneous Gas Distribution. arXiv, June 2018. 1806.06080.

H. Yan and R. A. Windhorst. Candidates of $\mathrm{z} \simeq 5.5-7$ Galaxies in the Hubble Space Telescope Ultra Deep Field. The Astrophysical Journal, 612(2):L93-L96, August 2004. doi: $10.1086 / 424690$.

T. Zafar, C. Péroux, A. Popping, B. Milliard, J.-M. Deharveng, and S. Frank. The ESO UVES advanced data products quasar sample. Astronomy 6 Astrophysics, 556:A141, August 2013. doi: 10.1051/0004-6361/201321154.

V. E. Zakharov and V. I. Karas. Nonequilibrium Kolmogorov-type particle distributions and their applications. Physics-Uspekhi, 56(1):49-78, January 2013. doi: 10.3367/ufne. 0183.201301c.0055.

J. Zhang, J.-L. Kuo, H. Liu, Y.-L. Sming T., K. Cheung, and M.-C. Chu. The Importance of Quantum Pressure of Fuzzy Dark Matter on Lya Forest. The Astrophysical Journal, 863(1):73, August 2018a. doi: 10.3847/1538-4357/aacf3f. 
J. Zhang, Y.-L. S. Tsai, J.-L. Kuo, K. Cheung, and M.-C. Chu. Ultralight Axion Dark Matter and Its Impact on Dark Halo Structure in N-body Simulations. The Astrophysical Journal, 853(1):51, January 2018b. doi: 10.3847/1538-4357/aaa485.

U.-H. Zhang and T. Chiueh. Evolution of linear wave dark matter perturbations in the radiation-dominated era. Physical Review D, 96(2), July 2017a. doi: 10.1103/physrevd. 96.023507 .

U.-H. Zhang and T. Chiueh. Cosmological perturbations of extreme axion in the radiation era. Physical Review D, 96(6), September 2017b. doi: 10.1103/physrevd.96.063522.

A. Zolotov, A. M. Brooks, B. Willman, F. Governato, A. Pontzen, C. Christensen, A. Dekel, T. Quinn, S. Shen, and J. Wadsley. Baryons Matter: Why Luminous Satellite Galaxies Have Reduced Central Masses. The Astrophysical Journal, 761(1):71, November 2012. doi: $10.1088 / 0004-637 x / 761 / 1 / 71$.

T. J. M. Zouros and D. M. Eardley. Instabilities of massive scalar perturbations of a rotating black hole. Annals of Physics, 118(1):139-155, March 1979. doi: 10.1016/ 0003-4916(79)90237-9. 


\section{Acknowledgements}

I wonder how many people can truly say that they love what they do and enjoy coming to work. I am probably one of only a few. This is mainly due to the freedom Professor Niemeyer gives every member of his group in order to conduct independent research to the best of our ability. All the experience, all the people I had the pleasure to meet during my four years as one of his PhD students, all the wonderful physical insights I was able to collect, all this was possible due to Jens' constant guidance and support. When I had questions or problems, he took his time to gradually introduce me to the concepts of numerical cosmology. Thank you very much! I am extremely happy that I can continue working with you in the years to come.

I also want to thank Laura Covi for her continued support over the last seven years - from my bachelors' all the way to my PhD, and the secretaries of the astrophysics department for always being available to provide practical help.

The group would not have been so family-like without all its members. Thank you all for your support, lively discussions, coffee times, trips, evening gatherings, and much more. I will always remember the good times we had. I also thank my family and friends for being there for me, and my girlfriend Tabea for her independence and grace.

Last but not least, I thank my committee members for their time in reviewing this work. 


\section{Publication List}

B. Schwabe and J. C. Niemeyer and J. F. Engels.

Simulations of solitonic core mergers in ultralight axion dark matter cosmologies.

Physical Review D, 94(4), August 2016. doi: 10.1103/physrevd.94.043513.

X. Du and C. Behrens and J. C. Niemeyer and B. Schwabe.

Core-halo mass relation of ultralight axion dark matter from merger history. Physical Review D, 95(4), February 2017. doi: 10.1103/physrevd.95.043519.

X. Du and B. Schwabe and J. C. Niemeyer and D. Bürger. Tidal disruption of fuzzy dark matter subhalo cores.

Physical Review D, 97(6), March 2018. doi: 10.1103/physrevd.97.063507.

J. Veltmaat and J. C. Niemeyer and B. Schwabe.

Formation and structure of ultralight bosonic dark matter halos.

Physical Review D, 98(4), August 2018. doi: 10.1103/physrevd.98.043509. 


\title{
Curriculum Vitae
}

\author{
name Bodo Schwabe \\ date of birth 5. January 1989, Lübeck \\ nationality German
}

1999 - 2008 Abitur, Leibniz Gymnasium Bad Schwartau

Advanced courses: mathematics, physics

2009 - 2012 Bachelor of Science - Physics, Georg-August-Universität Göttingen

Focus on theoretical physics/particle physics

2011 - 2014 Bachelor of Science - Mathematics, Georg-August-Universität Göttingen Focus on numerical mathematics

2012 - 2014 Master of Science - Physics, Georg-August-Universität Göttingen Focus on astroparticle physics/QFT/theoretical physics

since 2014 PhD student - Physics, Georg-August-Universität Göttingen

Focus on numerical cosmology/dark matter

2010 - 2014 undergraduate teaching assistant, Georg-August-University Göttingen tutor for various lectures and revision courses

2010 - 2014 summer student at DESY, Hamburg

since 2014 postgraduate research assistant, Georg-August-University Göttingen 